\title{
Simulations of the Karlsruhe Dynamo Using the Lattice-Boltzmann Method
}

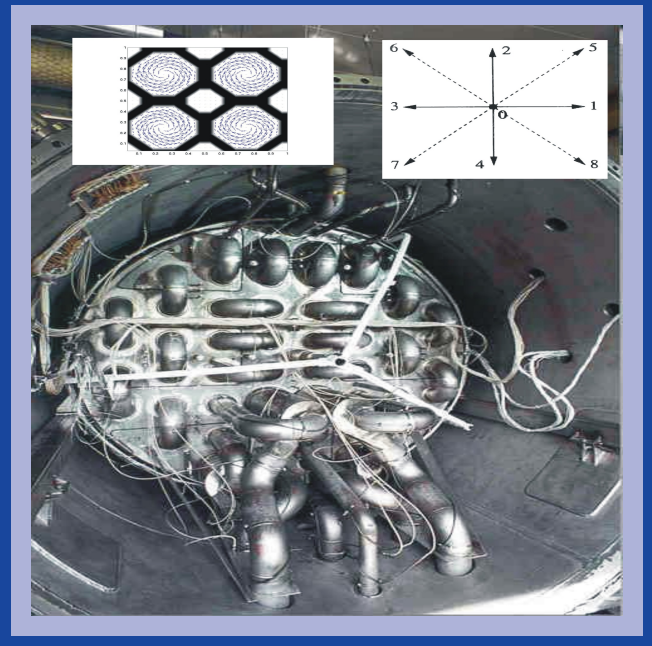

\section{Aveek Sarkar}

International Max Planck Research School

on Physical Processes in the Solar System and Beyond at the Universities of Braunschweig and Göttingen 


\title{
Simulations of the Karlsruhe Dynamo Using the Lattice-Boltzmann Method
}

\author{
Dissertation \\ zur Erlangung des Doktorgrades \\ der Mathematisch-Naturwissenschaftlichen Fakultäten \\ der Georg-August-Universität zu Göttingen
}

vorgelegt von

Aveek Sarkar

aus Kalkutta, Indien

Göttingen 2005 


\section{Bibliografische Information Der Deutschen Bibliothek}

Die Deutsche Bibliothek verzeichnet diese Publikation in der Deutschen Nationalbibliografie; detaillierte bibliografische Daten sind im Internet über http: / / dnb. ddb. de abrufbar.

D7

Referent: Prof. Dr Andreas Tilgner

Korreferent: Prof. Dr Ulrich Christensen

Tag der mündlichen Prüfung: July 4

Printed in Germany 
Dedicated to my parents

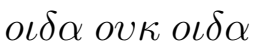





\section{Contents}

$\begin{array}{ll}\text { Abstract } & 7\end{array}$

1 Introduction 9

1.1 A Short Review of Dynamos . . . . . . . . . . . . . . . . . 10

1.1.1 Dynamo Equations . . . . . . . . . . . . . . . . . . . . . . . . 12

1.1 .2 Energy Balance . . . . . . . . . . . . . . . 13

1.2 Dynamo Experiments . . . . . . . . . . . . . . . . . 13

1.3 Numerical Methods . . . . . . . . . . . . . . . . . . . . . . . 14

1.3.1 Lattice Boltzmann Method . . . . . . . . . . . . . . . . . . . . . . 14

1.3.2 Spectral Method . . . . . . . . . . . . . . . . . . . 15

1.3.3 Our Hybrid Code . . . . . . . . . . . . . . . . . . . . 16

1.4 Motivation and Outline . . . . . . . . . . . . . . . . 16

2 The Karlsruhe Dynamo 19

2.1 Introduction . . . . . . . . . . . . . . . . . . . . . . 19

2.2 Dynamo . . . . . . . . . . . . . . . . . . . . . . . 19

2.2 .1 Basics of Dynamo $\ldots \ldots \ldots \ldots$

2.2 .2 Dynamo equations . . . . . . . . . . . . . 20

2.2 .3 Dimensionless Numbers . . . . . . . . . . . . . . . . . . 22

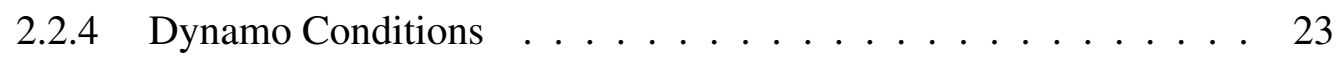

2.2.5 Mean-field model . . . . . . . . . . . . . . . . . . . . 24

2.3 Experimental Dynamos . . . . . . . . . . . . . . . . 27

2.3.1 Towards the laboratory implementation . . . . . . . . . . . . . 27

2.3.2 Karlsruhe Experiment . . . . . . . . . . . . . . . . . . 28

2.3 .3 G. O. Roberts Velocity . . . . . . . . . . . . . . . . . 28

2.3.4 Implementation in the experiment . . . . . . . . . . . 30

2.3.5 Experimental Results . . . . . . . . . . . . . . 32

3 Numerical Methods

3.1 Introduction . . . . . . . . . . . . . . . . 35

3.2 Lattice Boltzmann Method . . . . . . . . . . . . . . . . . . 35

3.2.1 Recovery of the Navier Stokes Equation . . . . . . . . . . . . 35

3.2 .2 Velocity Discretization . . . . . . . . . . . . . . . . 37

3.2 .3 Time Discretization . . . . . . . . . . . . . . . . . . . . 39

3.2 .4 Stability and Accuracy . . . . . . . . . . . . . . . . . . . . 40

3.2 .5 Boundary Condition . . . . . . . . . . . . . . 41 
3.3 Spectral Method . . . . . . . . . . . . . . . . . . . . 42

3.3.1 The Spectral Method to Solve Navier Stokes Equation . . . . . . 43

3.3.2 The Spectral Method to Solve the Induction Equation . . . . . . . 44

3.4 Advantages and Disadvantages of LBM and Spectral Method . . . . . . . 44

4 Numerical Experiments and code coupling $\quad 47$

4.1 Introduction . . . . . . . . . . . . . . . . . . . . 47

4.2 Numerical Experiments . . . . . . . . . . . . . . . . . . . . . . 47

4.2.1 Flow through a channel . . . . . . . . . . . . . 47

4.2.2 Flow past a circlular object . . . . . . . . . . . . . . 49

4.2 .3 Taylor-Couette Flow . . . . . . . . . . . . . . . 50

4.3 Coupling of Spectral and LBM codes . . . . . . . . . . . . 53

5 Numerical Simulations of the Karlsruhe Dynamo 57

5.1 Some previous calculations . . . . . . . . . . . . . 57

5.2 An analytical derivation of the wave . . . . . . . . . . . 58

5.3 Simulation of the modified velocity profile . . . . . . . . . . . . 60

5.4 Magnetic field oscillation in simulations . . . . . . . . . . . . . 61

5.5 Rotating wall simulation . . . . . . . . . . . . . . . 67

5.5.1 Subcritical onset . . . . . . . . . . . . . . . . 67

5.6 On the MHD instability of the G. O. Roberts flow . . . . . . . . . . 68

5.6.1 G. O. Roberts dynamo with changing superimposed perturbations 71

5.6 .2 Discussion . . . . . . . . . . . . . . . . 74

6 Summary and Outlook $\quad 77$

A Appendix: A Mean field view of the Karlsruhe Dynamo 79

$\begin{array}{lr}\text { Acknowledgements } & 85\end{array}$

$\begin{array}{lr}\text { Scientific Contributions } & 87\end{array}$

$\begin{array}{lr}\text { Lebenslauf } & 89\end{array}$ 


\section{Abstract}

The dynamo mechanism to generate magnetic fields of celestial bodies, including the Earth, by conversion of kinetic into magnetic energy is on the way to be understood. Several numerical simulations have shown the dynamo mechanism successfully. In recent years dynamo mechanism could be brought down to the laboratory level, on which selfsustained magnetic fields have been observed (fed by the kinetic energy of the fluid, as predicted in the theory). One of these successful laboratory experiments has been performed at Karlsruhe, Germany.

Even though the magnetic field is self-sustained in the experiment, certain magnetic field oscillations around its mean value during saturation are still to be interpreted.

Simulations of the dynamo effect require the simultaneous integration of the NavierStokes equation and of the induction equation of electrodynamics. We develop a hybrid method in which the Navier-Stokes equation is solved with a Lattice-Boltzmann method and the induction equation is treated with a spectral method.

Later, this hybrid code is used to simulate the Karlsruhe Dynamo experiment and in this thesis we suggest a possible cause of the afore mentioned magnetic field oscillations. 



\section{Introduction}

Since their availability high performance computers have opened up a new branch of science, with a particularly strong impact on physics. Until recently physics has divided itself happily into theoretical physics and experimental physics. Theoretical physics concerns itself with analytical solutions, expressing physical problems in terms of equations and solving it analytically. Analytical solutions, obviously, are not free from limitations. Complex equations, in the main, defy being solved analytically. Hence to solve them one needs to go through many approximations, and often neglects, in the process, quite a number of real-life situations. There may exist problems, which have no analytical solution at all. The second branch of physics, experimental physics in some difficult situation needs very accurate laboratory conditions to gain success. No wonder that some experiments turn out to be thwartingly expensive.

In the context of the advantages and disadvantages of these two branches of physics numerical simulation has given a remarkably welcome thrust to the subject. Categorized between theory and experiment, numerical simulation plays its role in overcoming the difficulties in both fields.

In numerical simulation one starts with complex equations which are solved with the aid of computers. Now that one is not called upon any longer to approximate these equations, all the real life parameters are conserved. Discrete algorithms for the equations must be provided, and then only the computer, comfortably reaches the solution, going step by step.

Since 1960 computer simulation is in progress. As time marches on and computers develop, computer simulation is becoming dominant almost in all branches of science. In recent years improved technology has given us good competent computers with the result that the demand for computer simulation is rising higher and higher. Nowadays even laboratory experiments are concurrently simulated numerically. Simulations of this kind, no doubt, come in useful to bring the experiments to perfection. Besides, many side-effects, because of cost effectiveness or inconvenience, are not possibly sighted during the experimental work. They can be easily and closely studied, however, with the help of numerical simulations. Moreover, the great advantage is reaped in that the simulation yields a trustworthy result because one is in a position to make a comparison between the experimental and the simulation result. Numerical simulation, thus, bridges the gap between theoretical physics and experimental physics.

This thesis targets on a laboratory experiment known as Karlsruhe Dynamo Experi- 
ment and its numerical simulation. The Karlsruhe Dynamo Experiment, though a success already, has some effects which were difficult to understand. But now they can be studied numerically. Even before setting up the experiment, numerical simulations helped to optimize various parameters.

\subsection{A Short Review of Dynamos}

Magnetism was already known during the time of Aristotle (384-322 BC). The Chinese are said to have built the first compass in the form of a loadstone spoon probably in the first century BC. It was Gilbert (1600) who concluded from his experiment that the terrestrial globe is a magnet. Since then it was a matter of question about what could be the origin of terrestrial magnetism. There were several theories to explain it. Meanwhile the solar magnetic field was discovered during the beginning of the last century. But till about 1950 there was no convincing theory which could explain the generation of this field. Any convincing theory had to explain not only the origin of the solar or terrestrial magnetic fields but also their dynamic behavior. Lamor (1919) was the first to suggest that motions in conducting fluids in the interior of the Sun could give an explanation for the observed magnetic field. He thus claimed the conversion of mechanical into electromagnetic energy as the basic mechanism for the solar magnetic field as in technical dynamos. Later on this process became known as dynamo process. Elsasser (1946) and Bullard (1949) among others were the first to investigate whether magnetic fields can be generated by the fluid motion within the Earth. Around the same time Frankel (1945), Gurevich and Lebedinskij (1945) had the idea that convective motions were responsible for the existence of the magnetic fields of the Earth and in sunspots. Parker (1955) in his famous paper first showed a generating mechanism of the magnetic field out of turbulence.

Celestial bodies which show magnetic field evolutions in time undergo the process of convection. As an example, the Earth has a solid, hot (about $6000^{\circ} \mathrm{K}$ ) inner core (made of nickel-iron alloy); above it lies a comparatively colder outer core (containing also a small portion of lighter elements like sulphur). There may be a convection process going on in the liquid outer core because it is heated from below by the solid inner core. There may exist another kind of convection as well: Pure iron of the liquid core solidifies at the bottom while the lighter constituents of the alloy are rejected, forming a buoyant alloy layer richer in the lighter constituents, which give rise to compositional convection. Without any rotation this would cause regular convection cells. However, under the influence of the rapid rotational motion, the buoyant convection develops a columnar pattern with axes parallel to the axis of rotation as in figure (1.1). Due to convection and rotation the net flow is somewhat helical inside the above mentioned columnar pattern (Busse,1971,1977).

The Earth, stars and other celestial bodies which show dynamic magnetic fields should go through the basic process named convection. In case of Sun-like stars there is an enormous source of heat from inside, which drives the convection in the outer layer. Whether it is the Sun or Earth, the convective fluid inside has to be sufficient electrically conductive to allow electric currents to flow. In magnetohydrodynamics there is a well-known theory named after Alfvén, which says magnetic field lines move along with the attached 


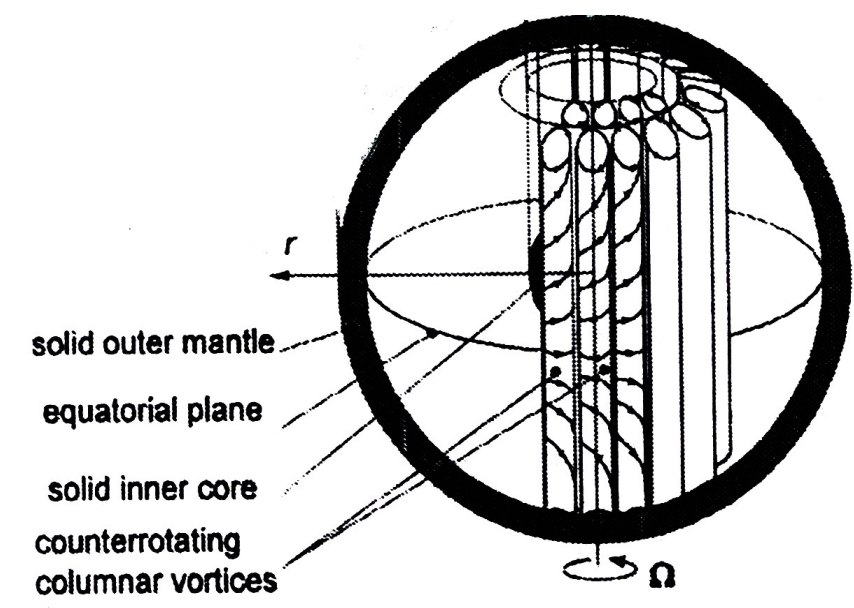

Figure 1.1: Convection cells inside the planet Earth

conducting fluid or magnetic field lines are dragged along with the moving fluid. This well-known theory is called Alfvéns frozen-in field. Following this and due to convective motion of the conducting fluid the magnetic field lines which are attached with it, get stretched and twisted. Provided that the Earths magnetic field is generated and maintained by a dynamo process, then the Alfv́en mechanism is essential for its realization.

\section{Zeldovich Effect}

Probably it will be worth mentioning here about Zeldovich's idea of stretch-twist-fold (STF) dynamos, (Vanishtein \& Zeldovich; 1972; Zeldovich, Ruzmaikin \& Sokoloff; 1983). Here an initial flux tube containing magnetic flux $\phi_{0}$ and energy $E_{0}$ is first considered as in figure (1.2). This flux tube is then stretched out, thus quadrupling the magnetic energy. It is, again, twisted and folded, to give a doubled tube shape. This has the energy $4 E_{0}$, and through a cross section of the doubled-up tube the flux is now $2 \phi_{0}$. If the process is similarly repeated, then, after $n$ reiterations, the energy will be $2^{2 n} E_{0}$ and the total flux through a cross section of the bundle of $2^{n}$ tubes will be $2^{n} \phi_{0}$. Hence it is clear that the growth rate is $\log 2$. This idea shows that STF can play a role for the evolution of magnetic fields. In case of homogeneous dynamos, this STF dynamo is realized by the fluid in motion.

The dynamical behavior of Earth's magnetic field, which is dipole-dominated, has apparently (from paleomagnetic data) reversed its polarity at irregular intervals between 10,000-100,000 years. Only in the last decade, numerical dynamo models, simulating 40,000 years in $2000 \mathrm{hrs}$ of CPU time, have been able to reproduce reversal of the field (Glatzmaier and Roberts 1995 a,b). Presently there are numerous geodynamo models which show the global changes of the Earth's magnetic field. For the Sun and other celestial bodies local evolutions can be seen using dynamo simulations. But before getting a detailed simulation, one needs to wait till higher computation power is achieved. 
(a)

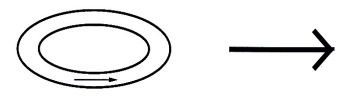

(b)

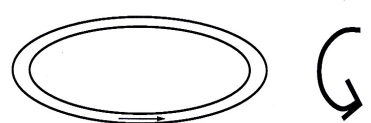

(c)

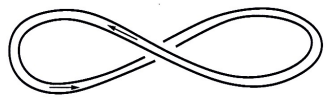

(d)

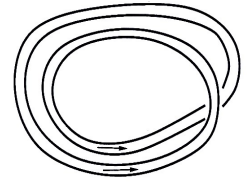

(e)

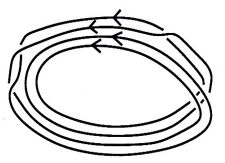

Figure 1.2: Zeldovich effect

\subsubsection{Dynamo Equations}

The equation relating to the evolution of the magnetic field is the magnetic induction equation which is non-linearly coupled to the Navier-Stokes equation, which describes the evolution of the velocity. Solving this system of non-linear equations is a formidable task.

In the kinematic dynamo problem it is assumed that the evolved magnetic field is so small that the Lorentz force generated by it is too weak to influence the velocity field. In this case the magnetic induction equation becomes decoupled from the fluid equation (Navier-Stokes) and the problem reduces to solving a linear partial differential equation for which we assume that the velocity field is fixed and known. In solving the magnetic induction equation, we find that for the production of dynamos the fluid must move faster in relation to the magnetic field than the field can diffuse away. A parameter which expresses the relation of advection to diffusion is called magnetic Reynolds number $\left(R_{m}\right)$. It depends on the fluid conductivity, the size of the system and the characteristic speed of the fluid. Dynamos typically have a critical magnetic Reynolds numbers above which dynamos work.

In this connection it is worth mentioning mean-field electrodynamics(MFE). The concept came from the turbulent fluctuation of the velocity field. MFE addresses the presence of fluctuations which are small compared to some mean value and performing statistical averages over the fluctuation quantities to determine the appropriate modifications to the equations. This theory explains so called alpha and beta effects. The alpha effect arises due to small-scale helical motions of turbulent flow and this effect produces currents antiparallel to the direction of the magnetic field line. If the helical motions of the fluctuations are correlated spatially, the net current generated can produce a large-scale magnetic 
field. The beta effect describes the increased transport of the magnetic flux due to turbulent stirring.

Once the magnetic field has grown enough, the kinematic dynamo approach is no more valid, leading to a non-linear dynamo problem. Because in that case the resulting Lorentz force is strong enough to influence the fluid flow and in this case we need to solve the full non-linear set of MHD equations. One anticipates that the flow will be modified in such a way as to limit the growth of the magnetic field. This "quenching" of field growth, a phenomenon also called "saturation", can be understood only in the framework of the nonlinear equations of MHD.

\subsubsection{Energy Balance}

The basic mechanism of the magnetic field generation in celestial bodies generally is accepted- it be a dynamo process, in which the magnetic field is maintained by convection of a highly electrically conducting fluid. But the details are less clear. For example, how much power is required to drive the dynamo process? The production of the magnetic energy mainly depends on two factors: work done by the Lorentz force and the Ohmic dissipation. Among these if the Ohmic dissipation term dominates the field is bound to decay. One the other hand work done by the Lorentz force is responsible for the field growth. In case of geodynamo it is still an enigma to predict quantitatively the power balance. Recently Christensen and Tilgner (2004) have shown a satisfying result to describe the power balance in the special case of the geodynamo.

\subsection{Dynamo Experiments}

One good way to understand the dynamo process is to perform laboratory realizations, with the need to generate a proper fluid geometry first of all. The next thing is to find out a proper conductor, which should allow us to reach the critical magnetic Reynolds number. Of course sufficiently high velocities of the liquid conductor also play a role to realize this condition. During the second half of the past century there were several attempts to produce dynamos in the laboratory. Some of them will be mentioned in chapter 2. Only towards the end of the last century it became possible to produce self-sustained dynamos in the laboratory. The Riga Dynamo Experiment (Gailitis et al.2003) in Latvia showed this for the Ponomarenko (1973) type dynamo in 1999 and in the same year the Karlsruhe Dynamo Experiment in Germany showed the same for G. O. Roberts (1972) dynamo.

The hydrodynamic Reynolds number $\left(R_{e}\right)$ for the Earth's outer core is of the order of $10^{9}$ which is difficult to reproduce in the laboratory. But fortunately the magnetic Reynolds number $\left(R_{m}\right)$ is expected to assume a much smaller value of the order of 500 . Certainly, even achieving this magnetic Reynolds number in the laboratory is a difficult job with the present-day technology. But even within these limitations self-sustained dynamos can be obtained and that is why the design of laboratory dynamos is an emerging field at present. Several conductors were tried until it was found that liquid sodium is 
much more advantageous than others. The conductivity of sodium suits well enough to reach the critical magnetic Reynolds number $\left(R_{m}\right)$. The Riga Dynamo Experiment followed the design of Ponomarenko where liquid sodium is driven helically in a cylinder and the resulting magnetic field showed an oscillatory growth as expected from theoretical calculations.

The Karlsruhe Dynamo Experiment is a bit different from the one in Riga. The magnetic field is dominated by its large-scale component, which extends throughout the entire cylindrical box and beyond. Here the fluid is driven through pipes and complex mechanical structures (it will be discussed in detail in Chapter 3), so that it can reproduce the G. O. Roberts velocity profile. The advantage of this experiment is that turbulence cannot change the overall velocity profile much as it is guided by the structures. In its saturation the Karlsruhe Dynamo shows some kind of magnetic field oscillations around its mean value. This in a strict sense is in conflict with G. O. Roberts dynamo solution. To get the physical reasons for these oscillations, one is provoked to do further experiments which can keep track of the velocity profile and the magnetic field together. One is encouraged also to do numerical simulations for an idea about the sources of these oscillations.

\subsection{Numerical Methods}

Some differential equations are difficult to solve, that is true. Substituting then the continuous problem by a discrete problem, this is called discretization. In numerical analysis errors are an important aspect to follow up, as for example discretization errors because the solution of the discrete problem does not coincide with the solution of the continuous problem. Discretization errors errors arise from deficiencies when a function of a continuous variable is replaced by a sequence of finite numbers in the computer, occupying for example nodal points in a lattice. Such deficiencies can usually be reduced by using a more finely spaced lattice, at the expense of increased computational costs. The magnitude of the discretization error, $e_{j}^{n}$, at the $(j, n)$-th node typically is written in terms of grid spacings, $\triangle x$ and $\triangle t$, and on the values of the higher-order derivatives at that node. In this thesis the Spectral Method and the Lattice Boltzmann Method (LBM) are used to solve dynamo equations. The spectral method is well known method whereas the LBM is a comparatively new one. We use the LBM to solve dynamo equations for the fact that it is a method which can help to implement complex boundaries, not counting the other advantage of this method that it is easy to implement.

\subsubsection{Lattice Boltzmann Method}

The lattice Boltzmann equation have a rather short history extending only over a decade or so and it has attracted much attention among physicists in various disciplines as an alternative and promising numerical scheme for simulating fluid flows and modelling physical processes in fluids. The reason is that the method has demonstrated its great potentials to study various complex systems where the application of other methods would be diffi- 
cult or impractical.

It has been a challenge for turbulence computations to capture the geometrical complexities of real life applications. Geometry is a major driver of complexity in these problems, as it selects the morphology and energetics of coherent structures sustaining the turbulence. LBM is well capable to tackle this kind of problems (Chen et al., 2003). The fundamental idea of the LBM is to construct simplified kinetic models that incorporate the essential physics of macroscopic or mesoscopic processes so that the macroscopic averaged properties obey the desired macroscopic equations. The basic premise for using these simplified kinematic-type methods for macroscopic fluid flows is that the macroscopic dynamics of a fluid is the result of the collective behavior of many microscopic particles in the system and that the macroscopic dynamics is not sensitive to the underlying details in microscopic physics (Kadanoff, 1986).

Historically, the idea of LBM came from Lattice Gas Automata (LGA), a discrete particle kinetics utilizing a discrete lattice and discrete time, where particle velocities are discrete as well. In general, a LGA consists of a regular lattice with particles residing on the nodes. A set of Boolean variables $n_{i}(\boldsymbol{x}, t)(i=1, \ldots ., N)$ describing the particle occupation is defined, where $N$ is the number of directions of the particle velocities at each node. These particles move from one node to the next over every time step. There they meet other particles resulting in collision. In collision particles change their velocity directions according to the collisional rules. To consider every individual particle is a bit expensive. So, in the statistical evolution of the LBM, deterministic particle occupation variables $n_{i}$ (Boolean variables) are replaced by single particle distribution function $f_{i}=\left\langle n_{i}\right\rangle$. This substitution eliminates noise due to the individual particle as well.

The key variable in LBM is the particle distribution function $f_{i}$. The time evolution of this function in the phase space (i.e. the space containing position, velocity and time) can be described using the Boltzmann equation. Again by integrating this equation one gets the hydrodynamic solution (as for example bulk velocity of the fluid, density etc.). LBM is involved to discretize the Boltzmann equation and then to integrate so that one arrives at the hydrodynamic solution. The collisional operator in the Boltzmann equation is replaced by the BGK (Bhatnagar,Gross,Krook;1954) approximation in our calculations.

\subsubsection{Spectral Method}

Spectral representations have been used for analytical studies of differential equations since the days of Fourier (1822). The idea of using them for numerical solutions of ordinary differential equations goes back at least to Lancozos (1938). Some present spectral methods can also be traced back to the "method of weighted residuals" (Finlayson and Seriven; 1966). Their current popularity for partial differential equations (PDE) dates back to the early 1970s. The major advance at that time was the pseudospectral approach of Kreiss and Oliger (1972), which, like most other spectral methods benefited greatly from the Fast Fourier Transformation (FFT) algorithm. 
Generally, PDEs are governing the physical processes, initial and boundary values are provided to the PDE. One then writes the input data (initial and boundary values) as a superposition of the basic waves of the PDE. In this form, the solution of the PDE is easily calculated. The Spectral method is based on this. The PDE is transformed into the Fourier domain using FFT algorithm and is solved in this domain. This is relatively easier than solving it in real space.

Previous dynamo simulations have mostly used spectral methods. There are three reasons why spectral methods are favoured when computing magnetic fields. Firstly, the boundary conditions at a conductor/insulator interface are nonlocal. They acquire simple expressions when the magnetic field is spectrally decomposed in a suitable set of basis functions provided that the fluid is confined to plane layers, infinite cylinders, or spheres. Secondly, the condition that there is no magnetic monopole can accurately be implemented particularly in the spectral method. The third advantage is, in the spectral mehod the numerical error is almost zero. Dynamo problems are basically dealing with the stability problem where a small amount of numerical errors can lead to a strongly divergent results. Experience suggests that it is important to use an accurate high order scheme to simulate the magnetic field, and that is why the spectral method is used.

\subsubsection{Our Hybrid Code}

The dynamo experiments which are running or being planned at the moment have outer boundaries which can be reasonably approximated by one of the geometries amenable to spectral methods. Mechanical structures inside the experimental cell which serve to drive or guide the flow of the liquid conductor are made of material of similar conductivity as the fluid. They thus constrain the flow but not the currents. As mentioned above, magnetic field simulation needs a high order scheme. But a low order scheme suffices for the velocity field simulation. We are therefore prompted to develop methods which use spectral methods for the equations of electrodynamics and the LBM for solving the Navier-Stokes equation. The two codes are coupled to simulate complete dynamos.

\subsection{Motivation and Outline}

It is now understood that many cosmic bodies posses magnetic fields and that they undergo a dynamic evolution. It is also understood that the behavior of the magnetic fields of these bodies are controlled by the dynamo process. But some years ago bringing down the dynamo into the laboratory was a great challenge. Now one sees magnetic field evolution in the laboratory dynamo, which of course helps to give us a clearer understanding about the physical process involved. Yet more is needed. Theory predicts that not a single, but numerous arrangements are capable of producing dynamos. That is why around the world different laboratories are making their efforts.

Once the basic phenomenon is understood we try to dig out more things, and find 
out many more unknowns. As the Karlsruhe Dynamo magnetic field is evolving, we still need to know the cause behind the evolution nature. There are two possibilities at present to explore these kinds of things either by performing some more experiments or by using numerical simulation. This thesis is concerned with the latter.

LBM has been a challenging numerical technique in recent years to simulate complicated fluid flow. Recently it has been extended to magnetohydrodynamic problems as well. So far the complete simulations of the Karlsruhe Dynamo experiments have neglected the complex mechanical structures, which, in principle, can have some role in the resulting magnetic field evolution by creating a boundary layer close to the wall. Small scale turbulence can create interesting phenomena in the magnetic field evolution. In particular one sees unexpected oscillations of the magnetic field around its mean value in the experiment. Our main aim is to numerically simulate the whole dynamo experiment with walls by utilizing the LBM and spectral hybrid code and to explore the results which are not yet understood from the experiment. In this thesis we mainly concentrate to simulate the Karlsruhe Dynamo Experiment. Mostly all the simulations are non-linear dynamo simulations. We try to see the Lorentz force back-reaction effect on the fluid flow. We try to explore the possible reasons behind the magnetic field oscillation in the experiment.

So far all the numerical-simulations on the Karlsruhe dynamo were done neglecting the complex mechanical structures. We develop a hybrid code using LBM and spectral method which can implement any kind of geometry for the fluid flow. The Lattice Boltzmann part of the code acts as a Navier-Stokes solver which gives fluid velocity $\boldsymbol{u}$ as a solution. This $\boldsymbol{u}$ is taken into the induction equation to solve it towards the magnetic field $\boldsymbol{B}$. Then the induction equation is solved using the spectral method. Once the output $(\boldsymbol{B})$ of the induction equation is obtained, we calculate the Lorentz force, which is added as an external force in the Navier-Stokes equation. A flow-chart is given in the figure (1.3).

We simulate the dynamo in a 3-dimensional periodic box with four cells kept inside. The grid resolution is varied as per requirement. We arrange our thesis in the following way:

In Chapter 2 we describe the basics of the dynamo theory and the Karlsruhe Dynamo Experiment. The results obtained from the experiment are also discussed in this chapter. In Chapter 3 we discuss the Numerical Methods which are involved in this thesis. Specially the LBM and the spectral method are also discussed in brief. In Chapter 4 we explain the way in which we have evolved our LBM Navier-Stokes solver. Here we show some of our test results. Later in this chapter we explain how the LBM code is coupled with the Spectral code. Further we try to show the speed and accuracy of our hybrid code. Chapter 5 is devoted to explain our simulation results. The different simulations and their outputs are tried to correlate with the experimental observations. In Chapter 6 we finish with a summary and outlook. 


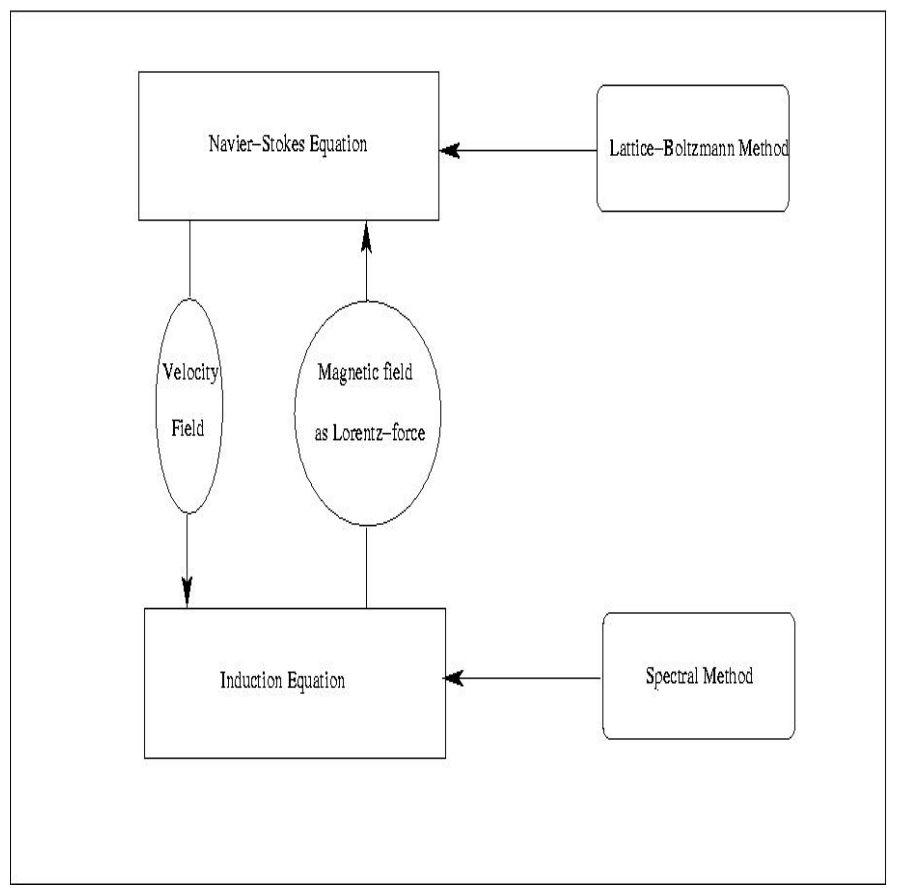

Figure 1.3: A flowchart of the hybrid code 


\section{The Karlsruhe Dynamo}

\subsection{Introduction}

The mathematical problem describing the generation of magnetic fields by motions in an electrically conducted fluid is called a dynamo problem. The magnetism of many naturally occurring bodies is attributed to the motion of conducting fluids in their interiors. These kinds of dynamo in general are called homogeneous dynamos. In the past decades there were many attempts to develop the homogeneous dynamos in the laboratory to see, what kinds of flow are exactly responsible for the dynamo action, and what happens when the newly produced Lorentz force acts back on the fluid motion. In the following section we shall try to describe the mathematical development of the dynamo problem and then the experimental dynamo (specially the Karlsruhe Dynamo Experiment) which will be later simulated numerically in this thesis.

\subsection{Dynamo}

The dynamo is the process which converts kinetic energy into magnetic energy. Most of the cosmic bodies are filled with conducting fluids which undergo continuous convective motion. As for example, the Earth has a solid inner iron core and a liquid core above it. The solid inner core is thought to be hotter (at $6000^{\circ} \mathrm{K}$ ) than the outer liquid core which helps the outer core to go through the convection process. There are however, materials which also contribute to this convection, known as material convection. Convection in general is a nonlinear phenomenon and eventually may give rise to turbulent motion in the fluid. Again in many celestial bodies this turbulence is responsible for bending and stretching any initial magnetic field in such a fashion that it grows in intensity. Altogether convection is a process which provides kinetic energy to the system and turbulence helps the energy to get transformed into the magnetic energy. In the case of the Sun the whole process is happening in the solar convection zone. Sometimes it is also the differential rotation of a cosmic body which contributes to the magnetic field modification, very common in the solar tachocline or, galactic disc.

\subsubsection{Basics of Dynamo}

To describe the idea of the dynamo theory qualitatively, it is important to introduce a spherical coordinate $(r, \theta, \phi)$ system. The $\phi$ component is called azimuthal coordinate, $\theta$ colatitude and $r$ radial distance. 
In this system the magnetic field can be split into polar and azimuthal parts (from the fact that a magnetic field has zero divergence):

$$
\boldsymbol{B}(\boldsymbol{r}, \theta, \phi)=\boldsymbol{B}_{p}+\boldsymbol{B}_{\phi}
$$

$\boldsymbol{B}_{p}$ is the polar part and can be written as $\boldsymbol{B}_{p}=\nabla \times \nabla \times(\boldsymbol{r} P)$, where $P(\boldsymbol{r}, \theta, \phi)$ is the poloidal scaler. $\boldsymbol{B}_{\phi}$ is the azimuthal or toroidal part and can be written as $\boldsymbol{B}_{\phi}=\nabla \times(\boldsymbol{r} T)$, where $T(\boldsymbol{r}, \theta, \phi)$ is the toroidal scaler. The azimuthal component $\boldsymbol{B}_{\phi}$ has no radial component whereas the polar component $\boldsymbol{B}_{p}$ can have all three components.

The production of both modes $\left(\boldsymbol{B}_{p}\right.$ and $\left.\boldsymbol{B}_{\phi}\right)$ are important in the dynamo theory. The initial poloidal field can easily be converted into the toroidal field by differential rotation, which is frequently present in cosmic bodies. In the core of the Earth, it is speculated that the Earth's solid core rotates faster than the surrounding liquid core. This faster rotating inner core can convert poloidal fields into toroidal fields. But this newly generated toroidal field is not visible from outside for example on the Earth's surface, where the poloidal field is visible only. Now it needs regeneration of the poloidal field out of the toroidal field to continue the process. To describe this Parker (1955) explained the turbulent dynamo theory.

The convection in the liquid core can regenerate a small scale poloidal field locally out of the toroidal field. This is called the $\alpha$ effect. The upward (or, downward) moving plasma (conducting fluid) blobs in convection stretch out the toroidal field in the upward (or, downward) direction due to flux-freezing. Taking rotation into account one would expect the motion to be helically up (or, down). The toroidal field lines can be twisted by such helical turbulent motions. Several such helical motions can give rise to the poloidal field altogether. The dynamo, explained above, is called $\alpha-\Omega$ dynamo. $\Omega$ term comes from the differential rotation. The poloidal and toroidal fields can be maintained by $\alpha^{2}$ dynamo as well, where turbulent motion is only responsible to produce both the fields.

\subsubsection{Dynamo equations}

As it is seen, the dynamo works when magnetic fields interact with complex velocity fields. So we need to formulate something which will couple these two fields. These coupled equations are the key MHD equations. The magnetic field behaviour can be well explained using Maxwell's equations.

$$
\begin{array}{r}
\nabla \cdot \boldsymbol{E}=\rho / \varepsilon, \\
\nabla \times \boldsymbol{E}=-\partial_{t} \boldsymbol{B}, \\
\nabla \cdot \boldsymbol{B}=0, \\
\nabla \times \boldsymbol{B}=\mu \boldsymbol{J}+\mu \varepsilon \partial_{t} \boldsymbol{E} .
\end{array}
$$

Here $\boldsymbol{E}$ is the electric field, $\boldsymbol{B}$ the magnetic field, $\boldsymbol{J}$ the electric current density and $\rho$ the electric charge density. We will assume here and below that the magnetic permeability 
$\mu$ and the dielectric constant $\varepsilon$ are constant, taking their free space values. The speed of light $c$ is defined by $c^{-2}=\varepsilon \mu$. From equation (2.1a) and (2.1d) one can have conservation of charge

$$
\partial_{t} \rho+\nabla \cdot \boldsymbol{J}=0
$$

We assume that velocities in the system under consideration should be much smaller than the speed of light $c$. We suppose the system has spatial length scale $\mathcal{L}$, temporal scale $\mathcal{T}$, and velocity scale $\mathcal{U}$ with

$$
\mathcal{U} \equiv \mathcal{L} / \mathcal{T} \ll c
$$

In this case equation (2.1b) gives the estimate

$$
E \sim \mathcal{U B}
$$

This approximation means displacement current $\mu \varepsilon \partial_{t} \boldsymbol{E}=c^{-2} \partial_{t} \boldsymbol{E}$ may be dropped from equation $2.1 \mathrm{~d}$ as it is negligible with a factor $\mathcal{U}^{2} / c^{2}$ smaller than $\nabla \times \boldsymbol{B}$. This leaves us with the reduced form of the Maxwell equations

$$
\begin{array}{r}
\nabla \cdot \boldsymbol{E}=\rho / \varepsilon, \\
\nabla \times \boldsymbol{E}=-\frac{\partial \boldsymbol{B}}{\partial t}, \\
\nabla \cdot \boldsymbol{B}=0, \\
\nabla \times \boldsymbol{B}=\mu \boldsymbol{J} .
\end{array}
$$

Using equation (2.1a) and (2.1d) gives the estimates

$$
\begin{gathered}
\rho \sim \varepsilon \mathcal{U B} / \mathcal{L} . \\
J \sim B / \mu \mathcal{L},
\end{gathered}
$$

which means equation (2.2) may be approximated by

$$
\nabla \cdot \boldsymbol{J}=0
$$

Ohms law in a moving medium can be written as

$$
\boldsymbol{J}=\sigma(\boldsymbol{E}+\boldsymbol{u} \times \boldsymbol{B})
$$

where $\sigma$ is the conductivity of the conductor and $\boldsymbol{u}$ is velocity. Combining (2.8), (2.5d) and $(2.5 \mathrm{~b})$ it can be written that the dynamic evolution of the magnetic field is

$$
\frac{\partial \boldsymbol{B}}{\partial t}=\nabla \times(\boldsymbol{u} \times \boldsymbol{B})+\frac{1}{\mu \sigma} \nabla^{2} \boldsymbol{B} .
$$

This is known as induction equation. The first term on the right hand side is called advection term. For an incompressible flow $(\nabla \cdot \boldsymbol{u}=0)$ one can write

$$
\nabla \times(\boldsymbol{u} \times \boldsymbol{B})=(\boldsymbol{B} \cdot \nabla) \boldsymbol{u}-(\boldsymbol{u} \cdot \nabla) \boldsymbol{B} .
$$


This indicates that the magnetic field is dragged in the fluid flow. The second term on the right hand side is called diffusion term. As it has been noticed already for sufficiently high conductivity magnetic field diffusion is negligible.

Along with the induction equation the Navier-Stokes equation is also required to describe the dynamo as a whole:

$$
\frac{\partial \boldsymbol{u}}{\partial t}+\boldsymbol{u} \cdot \nabla \boldsymbol{u}=-\frac{\nabla p}{\rho}+\nu \nabla^{2} \boldsymbol{u}+(\nabla \times \boldsymbol{B}) \times \boldsymbol{B} .
$$

This is basically a force balance equation, where $\boldsymbol{u}$ describes the fluid velocity, $-\nabla p$ is the pressure gradient force, $(\nabla \times \boldsymbol{B}) \times \boldsymbol{B}$ is another external force called Lorentz force and $\nu \nabla^{2} \boldsymbol{u}$ is called viscous force, where $\nu$ is known as kinematic viscosity.

The solution of the Navier-Stokes equation $\boldsymbol{u}$ is an input for the induction equation, and in the solution of the induction equation $\boldsymbol{B}$ calculates the Lorentz force which acts as an external force in the Navier-Stokes equation. Altogether, equations (2.9), (2.10) and $\nabla \cdot \boldsymbol{B}=0, \nabla \cdot \boldsymbol{u}=0$ are known as basic dynamo equations and this set of equations will be solved in the following part of the thesis.

It is better to mention that for a given velocity field, when the induction equation is solved alone along with the equation $\nabla \cdot \boldsymbol{B}=0$, the resulting dynamo is known as kinematic dynamo. On the other hand when all four equations are solved together the resulting dynamo is called a non-linear dynamo.

\subsubsection{Dimensionless Numbers}

Let us introduce a spatial scale length $\mathcal{L}$, velocity scale length $\mathcal{U}$ and magnetic scale length $\mathcal{B}$. These are some typical dimensions of the system. Then the ratio of the inertial term $(\boldsymbol{u} \cdot \nabla \boldsymbol{u})$ to the viscous term $\left(\nu \nabla^{2} \boldsymbol{u}\right)$ of equation (2.10) becomes

$$
\frac{\boldsymbol{u} \cdot \nabla \boldsymbol{u}}{\nu \nabla^{2} \boldsymbol{u}} \sim \frac{\mathcal{U} \frac{1}{\mathcal{L}} \mathcal{U}}{\nu \frac{1}{\mathcal{L}^{2}} \mathcal{U}}=\frac{\mathcal{U} \mathcal{L}}{\nu}=R_{e} .
$$

This ratio introduces a dimensionless number, known as hydrodynamic Reynolds number $\left(R_{e}\right)$. Similarly if we take the ratio of the advection term to the diffusion term of equation (2.9), we get

$$
\frac{\nabla \times(\boldsymbol{u} \times \boldsymbol{B})}{\frac{1}{\mu \sigma} \nabla^{2} \boldsymbol{B}}=\frac{\frac{1}{\mathcal{L}} \mathcal{U} \mathcal{B}}{\frac{1}{\mu \sigma} \frac{1}{\mathcal{L}^{2}} \mathcal{B}}=\mathcal{U} \mathcal{L} \mu \sigma=R_{m},
$$

which introduces another dimensionless number named as magnetic Reynolds number $\left(R_{m}\right)$. These are the main key parameters in dynamo problems. Writing down the dynamo equations in terms of $R_{e}$ and $R_{m}$ one gets the following non-dimensional equations: 


$$
\begin{array}{r}
\frac{\partial \boldsymbol{u}}{\partial t}+\boldsymbol{u} \cdot \nabla \boldsymbol{u}=-\nabla p+\frac{1}{R_{e}} \nabla^{2} \boldsymbol{u}+(\nabla \times \boldsymbol{B}) \times \boldsymbol{B}, \\
\nabla \cdot \boldsymbol{u}=0, \\
\frac{\partial \boldsymbol{B}}{\partial t}=\nabla \times(\boldsymbol{u} \times \boldsymbol{B})+\frac{1}{R_{m}} \nabla^{2} \boldsymbol{B}, \\
\nabla \cdot \boldsymbol{B}=0 .
\end{array}
$$

\subsubsection{Dynamo Conditions}

Let $V$ be the volume confined by the surface $S$, containing the conducting fluid, with a volume preserving flow field $\boldsymbol{u}(\boldsymbol{x}, t)$, conductivity $\sigma$ and diffusivity $\eta$. Suppose all space outside this volume, denoted by $\hat{V}$, is assumed to show the electromagnetic properties of vacuum. In general the definition of dynamos says the flow $\boldsymbol{u}(\boldsymbol{x}, t)$ is a dynamo if for some initial condition and magnetic diffusivity $\lambda>0$, the magnetic energy

$$
E_{m a g}=\frac{1}{2 \mu} \int_{V+\hat{V}}|\boldsymbol{B}|^{2} d V
$$

does not tend to zero as $t \rightarrow \infty$

There are two important conditions to complete the above mentioned definition.

First dynamo condition: All fields and currents must be created by fluid motion; none must be supplied by other sources external or internal.

Second dynamo condition: The fields and currents must persist indefinitely. The dynamo itself needs not be steady; $\boldsymbol{B}$ may be oscillatory, or even chaotic if $\boldsymbol{v}$ is chaotic.

The first dynamo condition demands that $\hat{\boldsymbol{B}}=O\left(r^{-3}\right)$ as $r \rightarrow \infty$, here $r$ is the distance from some origin in $V$, whereas $\hat{\boldsymbol{B}}$ denotes the magnetic field in the region $\hat{V}$. A commonly used idealisation is to assume space to be periodic in 1, 2 or 3 dimensions. In this case the integral giving the total energy is taken over one fundamental domain $V$. According to the second dynamo condition the total magnetic fluxes through the sides of periodicity are constant under the induction equation.

The evolution of the magnetic field in equation (2.9) is governed by the competition between diffusion and advection of the field. For vanishing velocity the magnetic field will disappear within a typical decay time $\mathcal{T}=\mu_{0} \sigma \mathcal{L}^{2}$, with $\mathcal{L}$ being a typical length scale of the system. On the other hand, the advection can lead to an increase of $\boldsymbol{B}$ within a kinematic time $\mathcal{T}_{k}=\frac{\mathcal{L}}{\mathcal{U}}$, with $\mathcal{U}$ being a typical velocity scale. If the kinematic time becomes smaller than the diffusion time, the net effect of the evolution can become positive, and hence the field will grow. The dimensionless number which compares the diffusion time scale with the kinematic time scale has been named before as magnetic Reynolds number $\left(R_{m}\right)$. Certainly this number has to be above its critical value to get a growth in the magnetic energy. 
The competition between field dissipation and production can also be understood in terms of the energy balance.

$$
\frac{d}{d t} \int \frac{\boldsymbol{B}^{2}}{2 \mu} d \boldsymbol{x}=-\int_{v} \frac{\boldsymbol{j}^{2}}{\sigma} d \boldsymbol{x}-\int_{v} \boldsymbol{u} \cdot(\boldsymbol{j} \times \boldsymbol{B}) d \boldsymbol{x} .
$$

This equation says that magnetic energy density $\boldsymbol{B}^{2} / 2 \mu$ is balanced by Ohmic heat production $\left(\boldsymbol{j}^{2} / 2 \sigma\right)$ in a unit volume and the work done by the Lorentz force is $(\boldsymbol{u} \cdot(\boldsymbol{j} \times \boldsymbol{B}))$ in a unit volume in a unit time. The second term on the right hand side is actually responsible for the dynamo action. It shows the conversion of mechanical energy into magnetic energy by deforming the conducting medium, which is always connected to the deformation of the magnetic field lines. If there is no motion of the conducting fluid, the magnetic energy eventually goes down to zero by Ohmic dissipation.

\section{Flow condition for Dynamo}

It is not that any sufficiently vigorous flow will result in dynamo action. There are certain restrictions which never allow magnetic fields to grow. In that connection Cowling's theorem should be first mentioned. Cowling's (1934) work has been extended by Backus \& Chandrasekhar (1956) and by Lortz (1968):

Cowling's Antidynamo Theorem:- A steady axisymmetric magnetic field cannot be maintained by dynamo action.

Toroidal Theorem:- Magnetic field can 'not be maintained by solenoidal motions without radial components. In other words any solution $\boldsymbol{B}$ of the induction equation will decay if the velocity field can be written in the form

$$
\boldsymbol{u}=\nabla \times \boldsymbol{r} \psi
$$

and if the diffusivity $\eta$ is a function of the radial co-ordinate, $r \equiv|\boldsymbol{r}|$ only.

This theorem has been proposed by Elsasser (1946), which has been proven rigorously by Bullard \& Gellman (1954).

\subsubsection{Mean-field model}

The mean-field concept starts with the basic dynamo equations (2.13a)-(2.13d) discussed earlier. In a turbulent medium, velocity and magnetic field vary irregularly. Basically because of the irregularity in the velocity field, the magnetic field also becomes irregular following the frozen-in field concept. Parker(1955)'s mathematical treatment on this was mostly based on intuitive arguments, but more formal and systematic approach was developed by Steenbeck, Krause \& Rädler (1966), Krause \& Rädler (1980). Let F be any fluctuating random field, the corresponding mean field $\bar{F}$ is then defined to be the expectation value of $\mathrm{F}$ in an ensemble of identical systems, and $F^{\prime}=F-\bar{F}$, is the fluctuating part. Here these hold the following relations: 


$$
\begin{array}{lrlrl}
F=\bar{F}+F^{\prime}, & \overline{\bar{F}}=\bar{F}, & \bar{F}^{\prime} & =0, \\
\bar{F}+G=\bar{F}+\bar{G}, & \overline{\bar{F}} \bar{G}=\bar{F}+\bar{G}, & \overline{\bar{F} G^{\prime}}=0,
\end{array}
$$

where $\mathrm{G}$ is another fluctuating field. The averaging operator commutes with the differentiation and integration operators in both space and time.

In a turbulent medium fluid velocity $\boldsymbol{u}$ can be split into two parts, 1 . Mean velocity $(\overline{\boldsymbol{u}})$, 2. Turbulent velocity $\left(\boldsymbol{u}^{\prime}\right)$ and in the same way the magnetic field can also be split into two parts.

Hence,

$$
\boldsymbol{u}=\overline{\boldsymbol{u}}+\boldsymbol{u}^{\prime} \quad \boldsymbol{B}=\overline{\boldsymbol{B}}+\boldsymbol{B}^{\prime}
$$

The turbulent motion $\boldsymbol{u}^{\prime}$ is assumed to possess a correlation time $\tau$ and length $\lambda$ small compared with the time scale $\mathcal{T}$ and length scale $\mathcal{L}$ of variation of $\overline{\boldsymbol{u}}$ and $\overline{\boldsymbol{B}} ; \tau$ is a mean time after which $\boldsymbol{u}^{\prime}$ has ceased to be correlated with its initial value, and $\lambda$ is a length comparable with the mean eddy size.

Substituting (2.15) into the induction equation (2.9) we get

$$
\begin{gathered}
\frac{\partial \overline{\boldsymbol{B}}}{\partial t}+\frac{\partial \boldsymbol{B}^{\prime}}{\partial t}=\nabla \times\left(\overline{\boldsymbol{u}} \times \overline{\boldsymbol{B}}+\boldsymbol{u}^{\prime} \times \overline{\boldsymbol{B}}+\overline{\boldsymbol{u}} \times \boldsymbol{B}^{\prime}+\boldsymbol{u}^{\prime} \times \boldsymbol{B}^{\prime}\right)+\frac{1}{\mu \sigma} \nabla^{2}\left(\overline{\boldsymbol{B}}+\boldsymbol{B}^{\prime}\right), \\
\frac{\partial \overline{\boldsymbol{B}}}{\partial t}=\nabla \times(\overline{\boldsymbol{u}} \times \overline{\boldsymbol{B}})+\nabla \times \boldsymbol{\epsilon}+\frac{1}{\mu \sigma} \nabla^{2} \overline{\boldsymbol{B}}
\end{gathered}
$$

where $\boldsymbol{\epsilon}=\overline{\boldsymbol{u}^{\prime} \times \boldsymbol{B}^{\prime}}$ is known as turbulent electromotiveforce (Krause, Rädler;1980). The equation (2.17) is almost like the induction equation except the term involving $\epsilon$, which is responsible for the mean field evolution. One thing to be pointed out here is that $\epsilon$ comes out in the induction just as Reynolds stresses appear in the averaged momentum equation.

Our prime interest is to see the evolution of $\overline{\boldsymbol{B}}$, having initial information of $\overline{\boldsymbol{B}}, \overline{\boldsymbol{u}}$ and $\boldsymbol{u}^{\prime}$. To do so the right hand side of equation (2.17) has to be expressed in terms of $\bar{B}, \boldsymbol{u}^{\prime}$ and $\overline{\boldsymbol{u}}$, hence $\epsilon$ can only be a functional of these properties. The initial step to be followed here is to evaluate $\boldsymbol{B}^{\prime}$ in terms of the above three quantities, and after subtracting (2.17) from (2.16) we get

$$
\frac{\partial \boldsymbol{B}^{\prime}}{\partial t}=\nabla \times\left(\boldsymbol{u}^{\prime} \times \overline{\boldsymbol{B}}+\overline{\boldsymbol{u}} \times \boldsymbol{B}^{\prime}+\boldsymbol{u}^{\prime} \times \boldsymbol{B}^{\prime}-\boldsymbol{\epsilon}\right)+\frac{1}{\mu \sigma} \nabla^{2} \boldsymbol{B}^{\prime} .
$$

The equation for $\boldsymbol{B}^{\prime}$ (2.18) is linear, with a source term involving $\overline{\boldsymbol{B}}$. Plainly $\boldsymbol{B}^{\prime}$ and so the e.m.f. $\boldsymbol{\epsilon}$ are linear functionals of $\overline{\boldsymbol{B}}$. Then the Taylor expansion of $\overline{\boldsymbol{B}}$ gives

$\epsilon_{i}=\left(\overline{\boldsymbol{u}^{\prime} \times \boldsymbol{B}^{\prime}}\right)_{i}=\alpha_{i j} \bar{B}_{j}+\beta_{i j k} \frac{\partial \bar{B}_{j}}{\partial x_{k}}+\gamma_{i j k l} \frac{\partial^{2} \bar{B}_{j}}{\partial x_{k} \partial x_{k}}+\ldots \ldots . .+a_{i j} \frac{\partial \bar{B}_{j}}{\partial t}+b_{i j k} \frac{\partial^{2} \bar{B}_{j}}{\partial x_{k} \partial t}+\ldots \ldots$.

here all quantities like $\alpha_{i j}, \beta_{i j k}, a_{i j}, b_{i j k}, \ldots \ldots$ are pseudo tensors depending on $\overline{\boldsymbol{u}}$ and $\boldsymbol{u}^{\prime}$. If the back-reaction of the magnetic field is taken into account, the velocity fields $\overline{\boldsymbol{u}}$ and 
$\boldsymbol{u}^{\prime}$ depend on $\overline{\boldsymbol{B}}$. It is worth noting that in this case the validity of (2.19) is not affected, whereas the tensors $\alpha_{i j}, \beta_{i j k}, \ldots .$. are now functionals of $\overline{\boldsymbol{u}}$ and $\boldsymbol{u}^{\prime}$ and $\overline{\boldsymbol{B}}$.

If in equation (2.19) only the spatial derivatives up to the first order are taken into account, then an error of the order $O\left\{\left(\frac{\lambda}{\mathcal{L}}\right)^{2}, \frac{\tau}{\mathcal{T}}\right\}$ must be expected. Hence

$$
\epsilon_{i} \simeq \alpha_{i j} \bar{B}_{j}-\beta_{i j k} \frac{\partial \bar{B}_{k}}{\partial x_{j}}
$$

is a sufficiently accurate description of the turbulent e.m.f. $\epsilon_{i}$.

Often the turbulent velocity field is assumed to be isotropic. For an isotropic turbulence all mean quantities derived from it remain unchanged if undergoing a rotation. Tensors with this property are called isotropic tensors. Expressing $\alpha_{i j}$ and $\beta_{i j k}$ of equation (2.20) in terms of isotropic tensors, one gets

$$
\alpha_{i j}=\alpha \delta_{i j} \quad \beta_{i j k}=\beta \epsilon_{i j k}
$$

Hence the turbulent e.m.f. in case of isotropic turbulence is

$$
\boldsymbol{\epsilon}=\overline{\boldsymbol{u}^{\prime} \times \boldsymbol{B}^{\prime}}=\alpha \overline{\boldsymbol{B}}-\beta \nabla \times \overline{\boldsymbol{B}} ;
$$

here $\alpha$ and $\beta$ are constant mean quantities determined by the turbulent velocity field $\boldsymbol{u}^{\prime}$ :

$$
\begin{array}{r}
\alpha=-\frac{1}{3} \tau \overline{\boldsymbol{u}^{\prime} \cdot(}\left(\nabla \times \boldsymbol{u}^{\prime}\right), \\
\beta=\frac{1}{3} \tau \boldsymbol{u}^{\prime 2} .
\end{array}
$$

The quantity $\boldsymbol{u}^{\prime} \cdot\left(\nabla \times \boldsymbol{u}^{\prime}\right)$ is called the helicity of the flow $\boldsymbol{u}^{\prime}$ (Moffatt 1969,1970). To have non-zero $\alpha$, overall helicity of the turbulent velocity field has to be non-zero.

At the end combining equations (2.17) and (2.22) we get the evolution equation of the mean field

$$
\frac{\partial \overline{\boldsymbol{B}}}{\partial t}=\nabla \times(\overline{\boldsymbol{u}} \times \overline{\boldsymbol{B}})+\nabla \times(\alpha \overline{\boldsymbol{B}})+(\beta+\lambda) \nabla^{2} \overline{\boldsymbol{B}}
$$

where $\lambda=\frac{1}{\mu \sigma}$ is called magnetic diffusivity. The term $\alpha \overline{\boldsymbol{B}}$, representing an electric field parallel to $\vec{B}$, enables the dynamo theory to escape from the antidynamo theorem. The quantity $\beta$ is an eddy diffusivity similar in its effects to the Ohmic diffusivity $\lambda$. It operates by mixing magnetic fields transported from neighboring regions; it does not destroy the resulting small-scale field inhomogeneities which have ultimately to be smoothed out by Ohmic diffusion. Its effect is to replace $\lambda$ by a total diffusivity $\lambda_{T}=\lambda+\beta$. 


\subsection{Experimental Dynamos}

Since 1960 there were several attempts to see dynamos in the laboratory, and in the past few years such dynamos have seen tremendous success. At the end of the last century there had been two laboratory dynamos which had shown the self sustained magnetic field. To be historically precise the work on experimental dynamos started from 1958, when Lenhart(1958) could observe the conversion of an applied polidal magnetic field component into a toroidal field, which is an important ingredient of the dynamo process. In the 1960s Willem Malkus and his co-workers pursued the possibility of magnetic field generation in a rotating cylinder filled with liquid sodium; but they did not succeed because of the unachievable magnetic Reynolds number required for the magnetic field to grow. Lowes \& Wilkinson $(1963,1968)$ avoided that problem but the properties of their dynamo differ significantly from the natural one. There were several other attempts after these to make the dynamo work in the laboratory. Two more past works resembling the content of this thesis also should also be mentioned here. Steenbeck et al.(1967) showed that sodium flow through two helically linked copper ducts can produce an e.m.f parallel to the applied magnetic field,and this e.m.f is proportional to the energy by which sodium is driven. In the same year Gailitis(1967) proposed an experiment with $12 \times 12$ spin-generators with the intention of simulating the $\alpha^{2}$ dynamo. It is seen that in most of these cases liquid sodium was used as conducting liquid.

Liquid Sodium has advantages over other liquid metals:

$\star$ It is an excellent conductor of heat.

$\star$ It has smallest diffusivity with $\frac{1}{\mu \sigma}=0.1 \mathrm{~m}^{2} / \mathrm{sec}$ at $120^{\circ}$ compared to other liquid conductors (mercury $0.8 \mathrm{~m}^{2} / \mathrm{sec}$, gallium $0.3 \mathrm{~m}^{2} / \mathrm{sec}$ ).

$\star$ Sodium is available in large quantities at low cost.

$\star$ Technology exists to pump and safely handle volumes of several cubic meters of liquid sodium.

The present generation of dynamos that worked successfully are the Karlsruhe Dynamo (Germany) and the Riga Dynamo (Latvia). The former is based on the theoretical work by G. O. Roberts (1972) and the latter on Ponomarenko's work (1973). The latest dynamo experiments are more closely to the natural dynamos, where dynamo actions will be created by convection in combination with rotation (eg. Madison Dynamo, Grenoble Dynamo).

\subsubsection{Towards the laboratory implementation}

The existing theoretical models (G. O. Roberts 1972, Ponomarenko 1973) could produce dynamos. But there was a problem implementing them in the laboratory. Even though magnetic diffusivity $\lambda=\frac{1}{\mu \sigma}$ was low enough for sodium, it was difficult to reach magnetic Reynolds numbers $\left(R_{m}\right)$ of the order of 10 or even 100 , which are expected to be required for a functioning dynamo. From the definition $R_{m}=\mu \sigma \mathcal{U L}$, it is clear that the velocity scale $\mathcal{U}$ or the length scale $\mathcal{L}$ are solely responsible for the value of this quantity for a particular material (as $\mu$ and $\sigma$ are material properties). So it needs quite a volumi- 
nous apparatus for the fluid to be driven with high velocity. Modern technology provides supports to achieve such critical magnetic Reynolds $\left(R_{m}\right)$ number.

\subsubsection{Karlsruhe Experiment}

The Karlsruhe Dynamo experiment reproduces qualitatively a simple spatially periodic velocity profile studied by G. O. Roberts (1972). G. O. Roberts showed analytically that this velocity structure can maintain dynamo action if certain conditions are fulfilled. Later Busse $(1975,1992)$ modified this profile, and thus brought the experiment into its final shape. These kinds of dynamos are called two-scale dynamos, the velocity field is a small-scale one whereas the magnetic field is dominated by its large scale component which extends throughout the experiment.

\subsubsection{G. O. Roberts Velocity}

G. O. Roberts velocity profile is a spatially periodic motion, which in principle can model convective cells. The flow field is a member of $A B C$ flows (Beltrami,1889,Arnold,1965, Childress, 1970).

$$
\boldsymbol{u}=(C \sin z+B \cos y, A \sin x+C \cos z, B \sin y+A \cos x)
$$

$A, B, C$ are constants, which have Beltrami property that $\nabla \times \boldsymbol{u}=k \boldsymbol{u}$, where $k$ is a constant. Making this flow $z$-independent i.e., assuming $C=0$ and taking $A=B=1$, one gets

$$
\begin{gathered}
\boldsymbol{u}=(\cos y, \sin x, \sin y+\cos x)=\left(-\partial_{y} \psi, \partial_{x} \psi, \psi\right), \\
\psi=\sin y+\cos x .
\end{gathered}
$$

This flow and flows similar to it are considered numerically by G. O. Roberts(1970) and analytically by Childress $(1967,1970)$ and G. O. Roberts(1972).

For the sake of convenience, the flow can be written in the form (after rotating axes, and rescaling)

$$
\begin{array}{r}
\boldsymbol{u}=\nabla \psi \times \boldsymbol{k}+\boldsymbol{k} w, \\
w i t h, \psi=A \sin a x \sin a y=w(x, y) A / C .
\end{array}
$$

Here $k$ is the unit vector along $z$ axis of the Cartesian co-ordinate, $\mathrm{A}$ and $\mathrm{C}$ being constants. Looking like figure (2.1), this kind of flow can be considered as a combination of several Ponomarenko flows (one in each cell), but every dynamo will be affected by all surrounding dynamos. The helicity of the flow is non-zero $(h \neq 0)$ in this kind of 


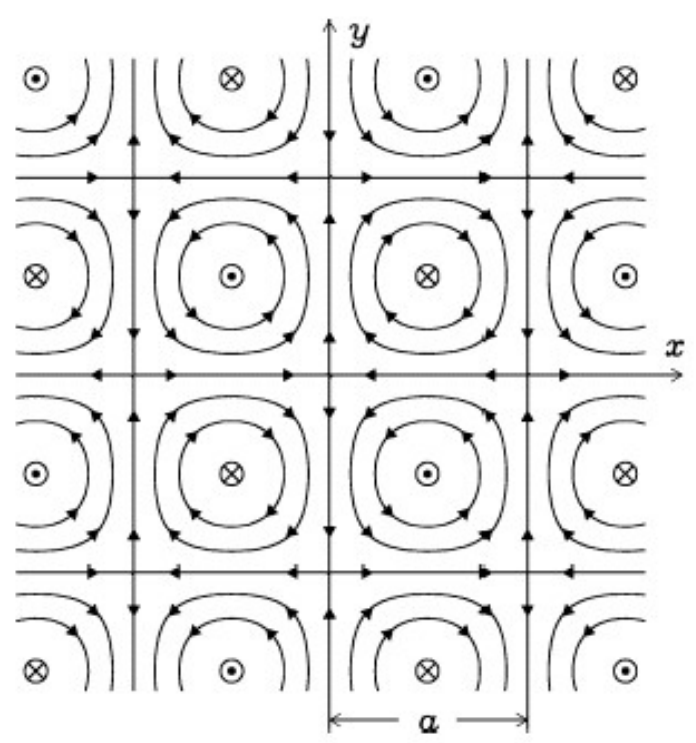

Figure 2.1: G. O. Roberts velocity profile: Projection of the streamlines in $(x, y)$-plane with the symbols $\otimes$ (down) and $\odot$ (up) for the $z$ component of the velocity.

flow. Since the velocity field (2.27) is $z$-independent and steady in time, the solution of equation $(2.13 \mathrm{c})$ can be obtained in the form

$$
\boldsymbol{B}=\boldsymbol{B}(x, y) \exp (i \gamma z+p t)
$$

where $\gamma$ is the real wave number of the magnetic field in $z$ direction and $p$ is the growth rate. The $z$-dependent solution is according to Cowling's theorem.

Bringing back the mean field concept here and assuming the mean flow is equal to zero, i.e. $\overline{\boldsymbol{u}}=0$ and $\boldsymbol{u}^{\prime}=\boldsymbol{u}$, we get

$$
\boldsymbol{B}=\overline{\boldsymbol{B}}+\boldsymbol{B}^{\prime}
$$

Following (2.29) and putting (2.28) into (2.9)we can derive the following two equations:

$$
\begin{array}{r}
\left(p+\gamma^{2} \lambda\right) \overline{\boldsymbol{B}}=i \gamma \boldsymbol{k} \times\left(\overline{\boldsymbol{u} \times \boldsymbol{B}^{\prime}}\right), \\
\left(p-\lambda \nabla^{2}\right) \boldsymbol{B}^{\prime}=\overline{\boldsymbol{B}} \cdot \nabla \boldsymbol{u}-w i \gamma \overline{\boldsymbol{B}}+\nabla \times\left(\boldsymbol{u} \times \boldsymbol{B}^{\prime}\right)-i \gamma \boldsymbol{k}\left(\overline{\boldsymbol{u} \times \boldsymbol{B}^{\prime}}\right) .
\end{array}
$$

Assuming that the fluctuating field $\boldsymbol{B}^{\prime}$ is small in comparison with the mean field, $\left|\boldsymbol{B}^{\prime}\right| \ll|\overline{\boldsymbol{B}}|$, one can neglect terms involving $\boldsymbol{B}^{\prime}$ on the right-hand side of (2.30b). This assumption is justified in the limit $\gamma^{2} \ll a^{2}, p \approx \gamma^{2}$. Hence from (2.30b) neglecting all other terms except the first (after first approximation) we get

$$
\boldsymbol{B}^{\prime}=\overline{\boldsymbol{B}} \cdot \nabla \boldsymbol{u} / 2 a^{2} \lambda
$$


Using this in equation (2.30a) to eliminate $\boldsymbol{B}^{\prime}$,

$$
\left(p+\gamma^{2} \lambda\right)=-i \gamma \frac{A C}{4 \lambda} \boldsymbol{k} \times \overline{\boldsymbol{B}},
$$

which is solved by

$$
\begin{gathered}
\overline{\boldsymbol{B}}=B_{0}\left(\boldsymbol{i}-\frac{\gamma A C}{|\gamma A C|} \boldsymbol{i} \boldsymbol{j}\right), \\
p=-\gamma^{2} \lambda+\frac{|\gamma A C|}{4 \lambda} .
\end{gathered}
$$

Here $i$ and $j$ are the unit vectors along $x$ and $y$ direction, respectively. If we define a toroidal magnetic Reynolds number $R_{m A}$ and a poloidal magnetic Reynolds number $R_{m B}$ as

$$
R m_{A}=\frac{|A|}{\lambda} \quad R m_{B}=\frac{|B|}{\gamma \lambda}
$$

respectively, then the necessary condition for the dynamo to work is $(p \geq 0)$

$$
R_{m A} R_{m B} \geq 4
$$

The dynamo action depends on the interaction between two modes of the velocity: the toroidal modes of length scale $a$ as expressed by $\psi(x, y)$, and the poloidal modes of length scale $\gamma$ as expressed by $w(x, y)$. The necessary condition for the dynamo to work holds the product of two magnetic Reynolds numbers.

The helical distortion of the original straight field line in the plane $z=0$ gives rise to an amplification of the field line in the planes above and below the plane $z=0$. Concurrently the distortions of the field lines in the plane $z=\pi / 2 \gamma$, which are orthogonal to the field in the plane $z=0$, give rise to a component in the $x$-direction wherever it is pushed downward. Thus the field at the lower level is enhanced. This is shown in figure(2.5).

\subsubsection{Implementation in the experiment}

Busse (1992) derived an approximate solution for the kinematic dynamo problem for a periodic velocity field ( as of G. O. Roberts) in a cylindrical confinement. In this analysis the periodic length $L=2 a$ is much smaller than the cylinder radius $r_{0}$ and its height $\mathrm{D}$. Based on this calculation the Karlsruhe Dynamo Experiment was modeled. Later Tilgner ( 1997,2002) and Rädler et al. (1998, 2002a) improved Busse's original model decisively with regard to conditions in the laboratory. They solved (2.13) with appropriate boundary conditions for current density and magnetic field.

In the Karlsruhe Experiment, liquid sodium is driven with three MHD pumps delivering flow rates up to $150 \mathrm{~m}^{3} / \mathrm{h}$ each through an array of 52 cells with square sections 
$0.21 \times 0.21 \mathrm{~m}^{2}$ in which sodium is forced to flow along helical streamlines. The outer dimensions of the cylinder containing the flow are approximately $0.94 \mathrm{~m}$ in radius and $0.9 \mathrm{~m}$ in height. All helices are right-handed, but up and down flows are alternating in neighbouring cells. Each cell contains two co-axial stainless-steel pipes and a bent metal sheet in the space between the two pipes in order to enforce the desired flow. A schematic view of the experimental setup is given in the figure (2.2).

The module is placed in a room in which the average magnetic field strength is less than

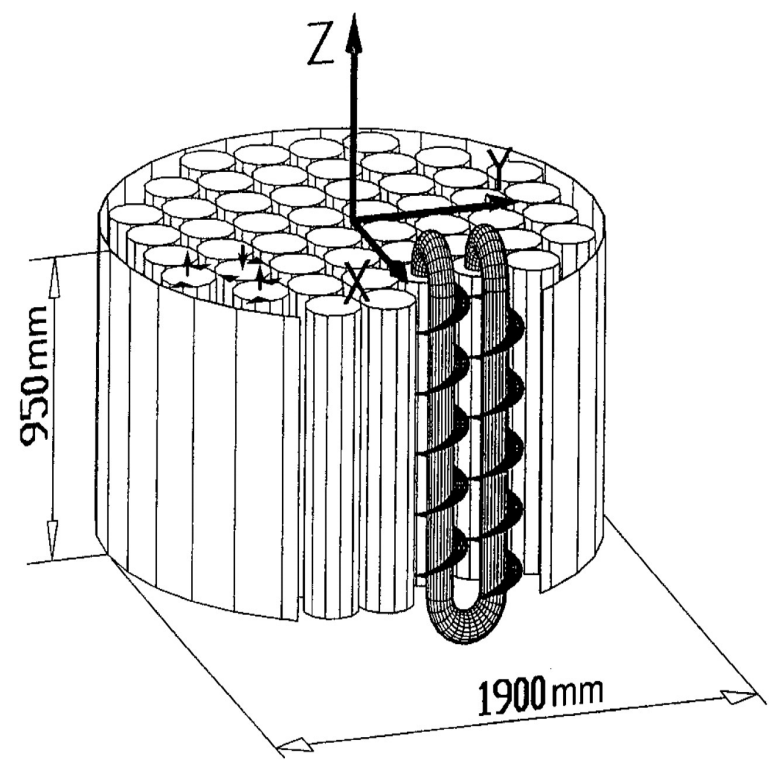

Figure 2.2: Experimental setup of the Karlsruhe Dynamo Experiment

0.5 Gauss. The dynamo's magnetic field is measured by six hall probes resolving less than 0.5 Gauss, two of them are fixed near to the equator of the cylinder, separated by $120^{\circ}$. The remaining are at variable positions along the cylinder axis between the center and the 'north pole'. Arrays of mobile compass needles are attached to a vertical wooden board, in order to obtain a qualitative impression of the structure of the generated magnetic field. Writing down the Roberts flow in the experimental condition we have

$$
\left(\begin{array}{c}
u_{x}=-u_{\perp} \frac{\pi}{2} \sin \left(\frac{\pi x}{a}\right) \cos \left(\frac{\pi y}{a}\right) \\
u_{y}=u_{\perp} \frac{\pi}{2} \cos \left(\frac{\pi x}{a}\right) \sin \left(\frac{\pi y}{a}\right) \\
u_{z}=-u_{\|}\left(\frac{\pi}{2}\right)^{2} \sin \left(\frac{\pi x}{a}\right) \sin \left(\frac{\pi y}{a}\right) .
\end{array}\right)
$$

Here $u_{\perp}$ is the average of the modulus of the velocity component in the $x y$ plane perpendicular to a line running from the center of a cell to its boundary taken over this line, eg. $\boldsymbol{u}_{\perp}$ is the average of $-u_{x}$ at $x=a / 2$ over $0 \leq y \leq a / 2$, or, of $u_{y}$ at $y=a / 2$ over $0 \leq x \leq a / 2$, and $u_{\|}$is the average of the modulus of $u_{z}$ over the cross section of a cell, eg. $0 \leq x, y \leq a$, that is,

$$
\begin{aligned}
& u_{\perp}=-\frac{2}{a} \int_{0}^{a / 2} u_{x}(a / 2, y) d y, \\
& u_{\|}=-\frac{1}{a^{2}} \int_{0}^{a} \int_{0}^{a} u_{z}(x, y) d x d y .
\end{aligned}
$$


Using $u_{\perp}$ and $u_{\|}$we define magnetic Reynolds numbers

$$
R_{m \perp}=\frac{u_{\perp} a}{\lambda} \quad R_{m \|}=\frac{u_{\|} a}{\lambda} .
$$

Finally we introduce volumetric flow rates

$$
V_{\perp}=\frac{a b}{2} u_{\perp} ; \quad V_{\|}=a^{2} u_{\|},
$$

where $b$ is a length characterising the pitches of the stream line. It is suggested that for the self excitation of the field the condition is

$$
R_{m \perp} \cdot R_{m \|} \geq \frac{32}{\pi}\left[1+\left(\frac{3.83}{\pi} \frac{d}{r_{0}}\right)^{2}\right]
$$

\subsubsection{Experimental Results}

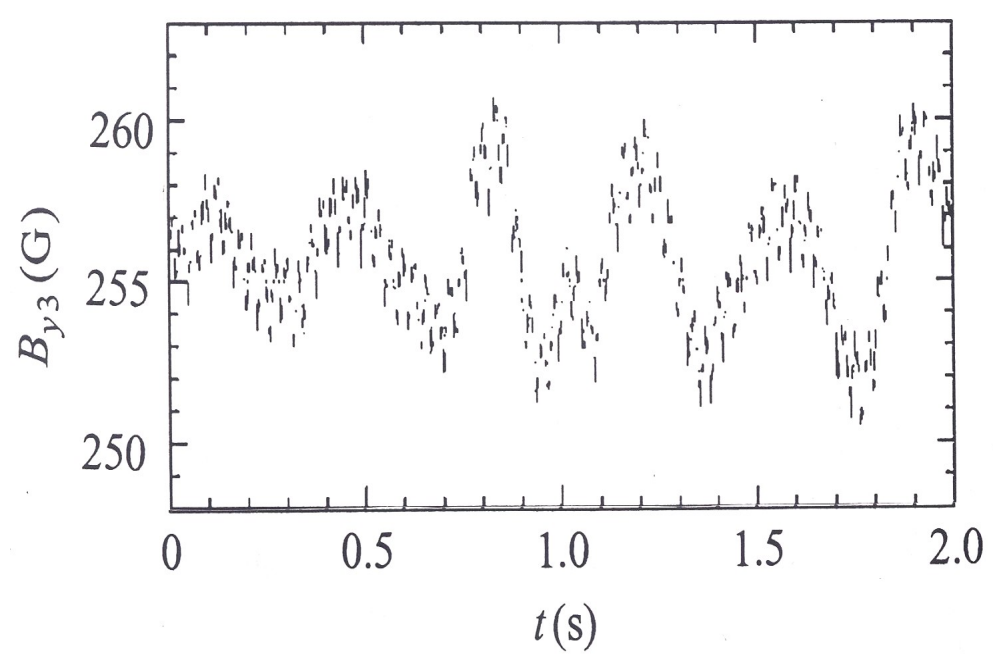

Figure 2.3: A sample time series showing the evolution of the $B_{y}$ component for a volumetric flow rate $115 m^{3} s-1$

Although the experimental results are explained elaborately by Müller \& Stieglitz (2002) and Müller, Stieglitz \& Horanyl (2004), we try to brief them here for the purpose of this thesis. The magnetic field evolution was observed for different volumetric flowrates $\dot{V}=95,102,106,111$, and $115 \mathrm{~m}^{3} \mathrm{~h}^{-1}$. Here the flow rates 95 and $102 \mathrm{~m}^{3} \mathrm{~h}^{-1}$ are subcritical for the onset of the dynamo, the flow rate $\dot{V}=106 \mathrm{~m}^{3} \mathrm{~h}^{-1}$ is about critical and the flow rates $\dot{V}=111 \mathrm{~m}^{3} h^{-1}$ and $115 \mathrm{~m}^{3} \mathrm{~h}^{-1}$ are supercritical. The saturated dynamo states are steady in the time average, but fluctuate about a mean value of the magnetic field. In figure (2.3) we show the time series data of the $B_{y}$ component recorded by one of the hall probes. This figure clearly indicates an oscillation of a time period around 


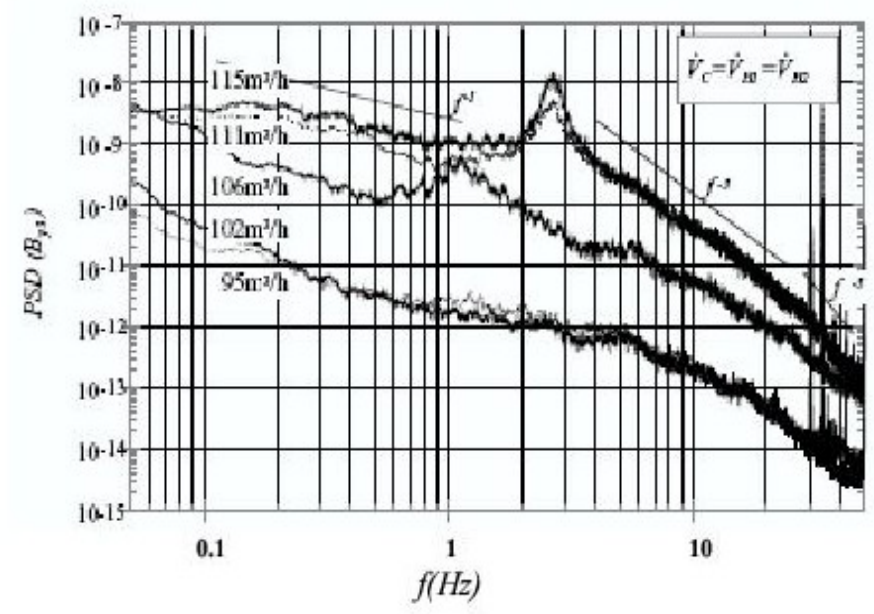

Figure 2.4: Power Spectral density (PSD) for the $y$ component of the magnetic field for five different volumetric flow rates

$0.4 \mathrm{sec}$. which corresponds a frequency of $2.5 \mathrm{~Hz}$. The power spectral density of the $B_{y}$ component is shown in the figure (2.4). This in the two supercritical states shows the characteristic spectral peak in the range $f \sim 2-3 \mathrm{~Hz}$. In the lower frequency range power shows a dependence on the frequency proportional to $\sim f^{-1}$ and the higher frequency range $3<f<20 H z$ spectral power decays nearly proportional to $\sim f^{-3}$; and beyond this range the power decays more rapidly. The other power spikes toward higher frequency $(f>30 H z)$ are due to experimental noise. The center frequency of the power peak $\left(f_{p}\right)$ seems to shift to lower frequencies for lower supercritical flow rates. It becomes broader and less pronounced when critical conditions are approached and finally disappears in the subcritical state. For the subcritical condition a broadband spectrum occurs without particular features.

The $f^{-1}$ relationship in the low frequency range seems to support the theoretical findings of Pouquet et al. (1976), who discovered a reverse energy cascade for a dynamo driven by an injection of helicity into the system at a particular frequency $f_{\text {in }}$ which is beyond the reverse cascade range. Some other dynamo experiments (Marie et al. 2002;Peffley et al. 2000) have noticed also the spectrum falling of as $f^{-1}$, but did not observed a subsequent spectral peak.

The spectral decay of the field intensity in the frequency range $f>f_{p}$ may be attributed to the interaction between the magnetic field and the local or non-local MHD wave. The spectral peak $f_{p}$ of the supercritical dynamo is not understood well yet. This peak in the PSD has not been seen in any other experiment. One doubts that the peak is due to the resonant interaction between the MHD wave and the smallest possible wavelength associated with the smallest structural length scale. It is not clear enough what kind of MHD wave can play a role in the PSD. Primarily one can think of the Alfvén wave, but 
this wave is damped in the liquid sodium on the length scale of about $0.1 \mathrm{~m}$., so the play of Alfvén wave is doubtful here. For clearer picture it is needed to perform some more experiments. Perhaps the numerical simulation of the whole dynamo experiment can give us some light as well. Later in this thesis we perform several numerical simulations in search of this power peak and its generation mechanism.

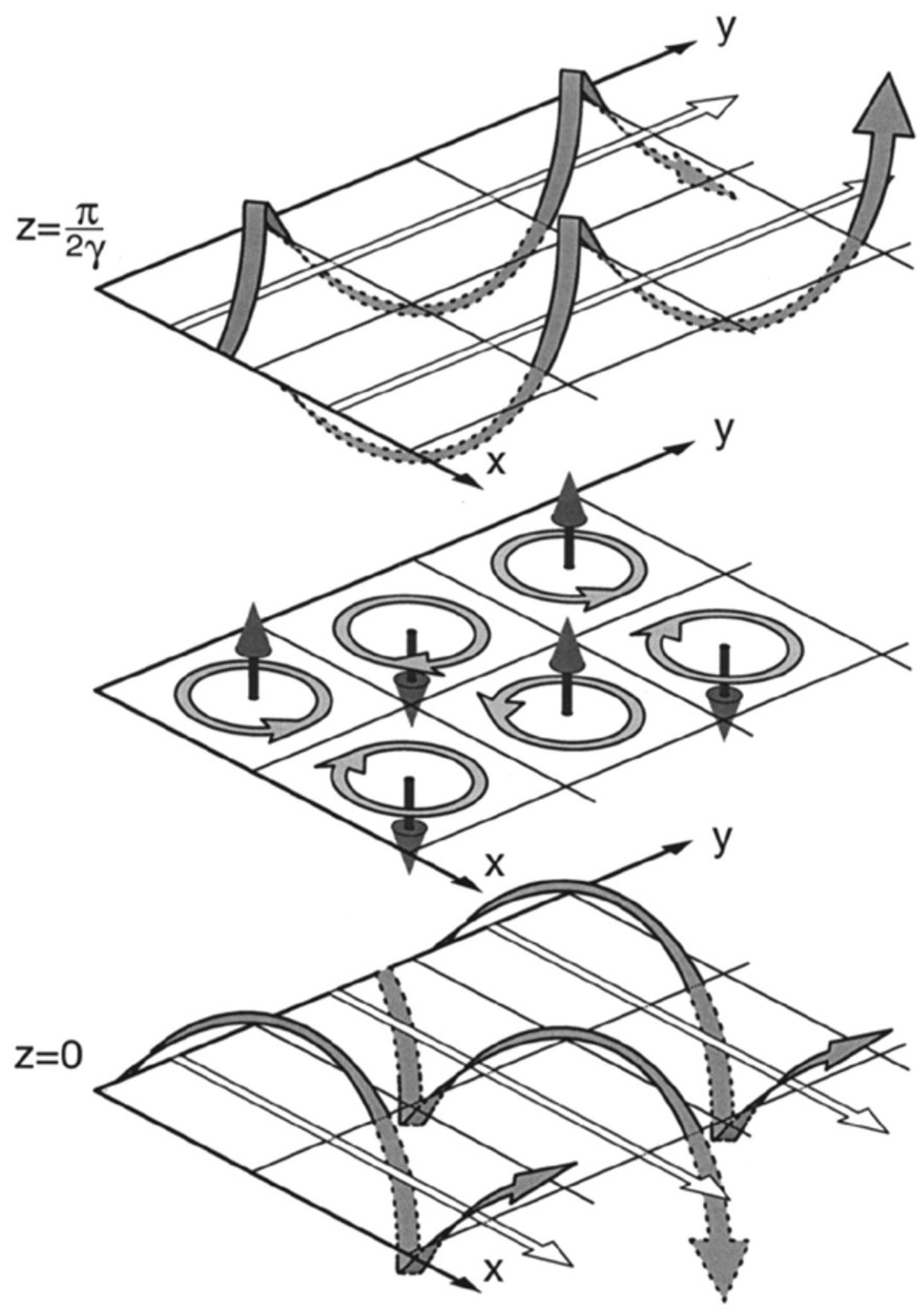

Figure 2.5: Magnetic field deformation in the G. O. Roberts Dynamo (Courtesy: Busse, 2000) 


\section{Numerical Methods}

\subsection{Introduction}

Numerical methods are the key parts of the intended computer simulations to solve the underlying partial differential equations (PDE) in a discrete way, which means there should be some method to discretize the original PDE. In our simulation we use the Lattice Boltzmann Method (LBM) and the Spectral Method to get the solution of Navier Stokes (NS) equation and induction equation respectively. Later these two methods are coupled to simulate complete non-linear dynamos. In the following section we first describe the LBM and then the Spectral Method along with their limitations and accuracies.

\subsection{Lattice Boltzmann Method}

In this thesis LBM is used to get the solution of the NS equation. Unlike other common methods (Finite Difference, Finite Volume etc.) it involves a discrete model concept. We start with the discrete Boltzmann equation more elaborately and after integration we arrive at the solution of the NS. The solution obtained in this way is almost incompressible, in the sense that the Mach-number $(M a)$, which is a ratio of the bulk velocity of the fluid to the sound velocity is limited up to 0.1 .

\subsubsection{Recovery of the Navier Stokes Equation}

The idea of the LBM originates from the kinetic theory of an ideal gas and the variable to be considered will be the probability distribution function (PDF) in the phase space. The notation of the PDF in general is $f(\boldsymbol{r}, \boldsymbol{v}, t)$, where $\boldsymbol{r}$ represents the space co-ordinate, $\boldsymbol{v}$ represents velocity and $t$ is time. One tries to see the evolution of the gas particles with the help of this PDF where $f(\boldsymbol{r}, \boldsymbol{v}, t)$ signifies the number of particles in a phase space $(d \boldsymbol{r} d \boldsymbol{v})$ at time $t$. The transport equation of this PDF can be expressed as

$$
\partial_{t} f+\boldsymbol{v} \cdot \nabla \boldsymbol{r} f+\frac{\boldsymbol{F}}{m} \cdot \nabla \boldsymbol{v} f=\left(\partial_{t} f\right)_{\text {coll }}
$$

where $\partial_{t} f$ signifies the time derivative, $\nabla \boldsymbol{r}$ signifies derivative over space and $\nabla \boldsymbol{v}$ derivative over individual particle velocity. $\frac{\boldsymbol{F}}{m}$ is the acceleration of particles, as given by the ratio of external force $(\boldsymbol{F})$ to mass $(m)$. The non-linear term on the right hand side represents the collision which will be expressed explicitely as follows: 
BGK approximation : In the simplest case, binary collision assumption can be made for the collisional term on the right hand side of equation (3.1). To consider multi-particle interaction one can replace the right hand side with the BGK (Bhatnagar,Gross,Krook;1954) approximation. In order to explain this approximation in simple language, if we start from any distribution function (near equilibrium), we expect it to relax to the Maxwellian distribution. Suppose $f$ is the initial distribution function and $f^{0}$ is the Maxwellian distribution function. Assuming $\tau$ as the relaxation time for $f$ to become $f^{0}$, according to BGK approximation the collisional term can be written as,

$$
\left(\partial_{t} f\right)_{c o l l} \approx-\frac{f-f^{0}}{\tau}
$$

where,

$$
f^{0}=n\left(\frac{m}{2 \pi \theta}\right)^{\frac{3}{2}} \exp \left(-\frac{m}{2 \theta}(\boldsymbol{v}-\boldsymbol{u})^{2}\right)
$$

Here $n$ is the particle density and $\theta=k T$ with $k$ as Boltzmann constant and $T$ as temperature. $\boldsymbol{v}$ is the velocity of a single particle and $\boldsymbol{u}$ is the macroscopic velocity. Hence $\boldsymbol{u}=\langle\boldsymbol{v}\rangle$. Inserting (3.2) in (3.1) we get the Boltzmann equation with the BGK approximation

$$
\partial_{t} f+\boldsymbol{v} \cdot \nabla \boldsymbol{r} f+\frac{\boldsymbol{F}}{m} \cdot \nabla \boldsymbol{v} f=-\frac{f-f^{0}}{\tau} .
$$

External forces : For $\boldsymbol{F} \neq 0$ it needs the knowledge of $\nabla \boldsymbol{v} f$. Following He et al.(1998) we set

$$
\nabla \boldsymbol{v} f \approx \nabla \boldsymbol{v} f^{0}
$$

assuming that $f$ and $f^{0}$ are chosen sufficiently close to eachother. In (3.4) if one modifies the equilibrium distribution function with

$$
\tilde{f}^{0}=f^{0}\left(\boldsymbol{v}-\frac{\boldsymbol{F}}{m} \tau\right)
$$

which already involves the external forcing term, the modified version of the Boltzmann equation becomes

$$
\partial_{t} f+\boldsymbol{v} \cdot \nabla \boldsymbol{r} f=-\frac{f-\tilde{f}^{0}}{\tau}
$$

This approximation introduces an error in the Boltzmann equation, which is of order $\left(\frac{|\boldsymbol{F}|}{m} \frac{\tau}{|\boldsymbol{v}|}\right)^{2}$. Hence,

$$
\tilde{f}^{0}=f^{0}\left(\boldsymbol{v}-\frac{\boldsymbol{F}}{m} \tau\right)=f^{0}-\frac{\boldsymbol{F}}{m} \tau \nabla \boldsymbol{v} f^{0}+O\left(\frac{|\boldsymbol{F}|}{m} \frac{\tau}{|\boldsymbol{v}|}\right)^{2},
$$

where

$$
\frac{F}{m} \frac{\tau}{|v|} \lesssim \frac{v \tau}{L}=\frac{\lambda}{L}
$$


after dimensional analysis.

Equation (3.8) says the error is of the order of the Knudsen Number $\left(K_{n}\right)$ as given by the ratio of the molecular mean free path length $(\lambda)$ to a respective physical length scale $(L)$.

So the modified Boltzmann equation gets the form

$$
\partial_{t} f+\boldsymbol{v} \nabla \boldsymbol{r} f+\frac{\boldsymbol{F}}{m} \cdot \nabla \boldsymbol{v} f=-\frac{f-f^{0}}{\tau}+O\left(K_{n}\right) .
$$

Now after integrating one arrives at the hydrodynamic solution

$$
\begin{gathered}
\int f d \boldsymbol{v}=\int f^{0} d \boldsymbol{v}=\int \tilde{f}^{0} d \boldsymbol{v}=\int f^{0} d\left(\boldsymbol{v}-\frac{\boldsymbol{F}}{m} \tau\right)=n, \\
\int \boldsymbol{v} f d \boldsymbol{v}=\int \boldsymbol{v} f^{0} d \boldsymbol{v}=\int \boldsymbol{v} \tilde{f}^{0} d \boldsymbol{v}=\int \boldsymbol{v} f^{0} d\left(\boldsymbol{v}-\frac{\boldsymbol{F}}{m} \tau\right)=n \boldsymbol{u}+n \frac{\boldsymbol{F}}{m} \tau ;
\end{gathered}
$$

here $n$ is the particle density and $\boldsymbol{u}$ is the bulk velocity of the fluid.
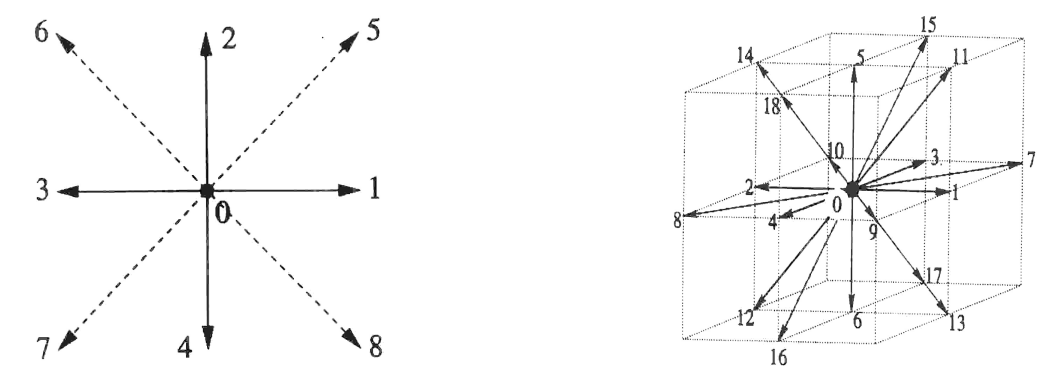

Figure 3.1: Discrete Velocities $\boldsymbol{v}_{i}$ of the Lattice Boltzmann Method, D2Q9 \& D3Q19

\subsubsection{Velocity Discretization}

LBM is initiated by the Lattice-Gas Cellular Automata, where the physical space is gridded and every grid point is populated by discrete particles. Each particle can have discrete possible velocities as in figure(3.1). For rectangular grids there are 9 possible velocities in two-dimensional cases and 19 possible velocities in three-dimensional cases. It is seen that these are the optimized number of velocities from the computational cost of view and to reproduce the fluid behavior. Particles hop from one grid point to the next over every time step; on the new grid point they meet other particles and following normal elastic collisional rules they choose their next destination.

Exactly the same kind of velocity discretization is adopted in LBM. In effect, the LBM corresponds to the following formal discretization in phase space of the Boltzmann equation: 


$$
\begin{aligned}
& f \longrightarrow f_{i} \\
& \boldsymbol{v} \longrightarrow \boldsymbol{v}_{i} \\
& f^{0} \longrightarrow f_{i}^{0}
\end{aligned}
$$

Discretization of $f^{0}$ is inspired by the following isothermal and low Mach-number approximation.

$$
\begin{aligned}
f^{0} & =n\left(\frac{m}{2 \pi \theta}\right)^{\frac{3}{2}} \exp \left(-\frac{m}{2 \theta}(\boldsymbol{v}-\boldsymbol{u})^{2}\right) \\
& =n\left(\frac{m}{2 \pi \theta}\right)^{\frac{3}{2}} \exp \left(-\frac{m}{2 \theta} \boldsymbol{v}^{2}\right) \exp \left(-\frac{m}{2 \theta}-2 \boldsymbol{v} \boldsymbol{u}+\boldsymbol{u}^{2}\right) \\
& =n\left(\frac{m}{2 \pi \theta}\right)^{\frac{3}{2}} \exp \left(-\frac{m}{2 \theta} \boldsymbol{v}^{2}\right)\left\{1+\frac{m}{\theta} \boldsymbol{v} \boldsymbol{u}-\frac{m}{2 \theta} \boldsymbol{u}^{2}+\frac{m^{2}}{2 \theta^{2}}(\boldsymbol{v} \boldsymbol{u})^{2}+O\left(M a^{3}\right)\right\}
\end{aligned}
$$

with Mach number $M a=\frac{|\boldsymbol{u}|}{c}$ and isothermal sound-speed $c=\sqrt{\frac{3 k T}{m}} ; k$ is the Boltzmann constant.

Equation (3.12) indicates that hydrodynamic equations can be recovered up to $O\left(M a^{2}\right)$ using this discretization. From (3.10) and (3.11) it is clear that the general integration to be done to derive the Navier-Stokes from Boltzmann equation is

$$
\left.\int d \boldsymbol{v} P_{n}(\boldsymbol{v}) n\left(\frac{m}{2 \pi \theta}\right)^{\frac{3}{2}} \exp \left(-\frac{m}{2 \theta}\right) \boldsymbol{v}^{2}\right) .
$$

Here $P_{n}(\boldsymbol{v})$ is a polynomial of order $n$ in the components of $\boldsymbol{v}$. The above integral can be evaluated, if $P_{n}(\boldsymbol{v})$ is known for a finite number of vectors $\boldsymbol{v}$, and in that case all the integrals are replaced by sums:

$$
\begin{aligned}
& \int d \boldsymbol{v} f^{0} P_{n}(\boldsymbol{v}) \\
& \approx n\left(\frac{m}{2 \pi \theta}\right)^{\frac{3}{2}} \int d^{3} \boldsymbol{v} \exp \left(-\frac{m}{2 \theta} \boldsymbol{v}^{2}\right)\left\{1+\frac{m}{\theta} \boldsymbol{v} \boldsymbol{u}-\frac{m}{2 \theta} \boldsymbol{u}^{2}+\frac{m^{2}}{2 \theta^{2}}(\boldsymbol{v} \boldsymbol{u})^{2}\right\} P_{n}(\boldsymbol{v}) \\
& \approx n\left(\frac{m}{2 \pi \theta}\right)^{\frac{3}{2}} \sum_{i} w_{i}\left\{1+\frac{m}{\theta} \boldsymbol{v}_{i} \boldsymbol{u}-\frac{m}{2 \theta} \boldsymbol{u}^{2}+\frac{m^{2}}{2 \theta^{2}}\left(\boldsymbol{v}_{i} \boldsymbol{u}\right)^{2}\right\} P_{n}\left(\boldsymbol{v}_{i}\right) \\
& =\sum_{i} f_{i}^{0} P_{n}\left(\boldsymbol{v}_{i}\right)
\end{aligned}
$$

Here $\boldsymbol{v}_{i}$ are the velocity vectors for which the Boltzmann equation is integrated, $w_{i}$ are the quadrature weights and $f_{i}^{0}$ is a discretized equilibrium distribution function for the LBM. Now our task is to properly specify the abscissas of the quadrature (3.14), or, in other words, the 'structure' or 'symmetry' of the lattice.

For that we take help of Gauss quadrature and determine the velocities $\boldsymbol{v}_{i} \mathrm{~s}$ and $w_{i} \mathrm{~s}$ for the 2D case: 


$$
\begin{gathered}
\boldsymbol{v}_{i}=c \cdot\left\{\begin{array}{cc}
(0,0), & i=0 \\
\left(\sin \left(\frac{i-1}{2}\right) \pi, \cos \left(\frac{i-1}{2}\right) \pi\right), & i=1,2,3,4 \\
\sqrt{2}\left(\sin \left(\frac{2 i-1}{4}\right) \pi, \cos \left(\frac{2 i-1}{4}\right) \pi\right), & i=5,6,7,8
\end{array}\right. \\
w_{i}= \begin{cases}\frac{4}{9}, & i=0 \\
\frac{1}{9}, & i=1,2,3,4 \\
\frac{4}{9}, & i=5,6,7,8\end{cases}
\end{gathered}
$$

In case of 3D Gauss quadrature the number of $3^{3}=27$ velocities is overestimated, while the optimized number which can reproduce Navier-Stokes with stability is a model with 19 velocities. They can be written down as

$$
\begin{gathered}
\boldsymbol{v}_{i}=c \cdot\left\{\begin{array}{cl}
(0,0,0), & i=0 \\
( \pm 1,0,0),(0, \pm 1,0),(0,0, \pm 1), & i=1 \ldots .6 \\
(0, \pm 1, \pm 1),( \pm 1,0, \pm 1),( \pm 1, \pm 1,0), & i=7 \ldots .18
\end{array}\right. \\
w_{i}= \begin{cases}\frac{1}{3}, & i=0 \\
\frac{1}{18}, & i=1 \ldots .6 \\
\frac{1}{36}, & i=7 \ldots .18\end{cases}
\end{gathered}
$$

\subsubsection{Time Discretization}

Assuming, again, the external forcing term to be implicit in $f^{0}$, we now want to discretize the equation

$$
\frac{D}{D t} f_{i}=\partial_{t} f_{i}+\boldsymbol{v}_{i} \partial \boldsymbol{r} f_{i}=-\frac{f-\tilde{f}_{i}^{0}}{\tau}
$$

This is a first-order partial-differential equation and for the solution it needs local information. The most natural method to do this is the finite volume method. Since only the difference $\boldsymbol{v}-\boldsymbol{u}$ occurs in the continuous equilibrium distribution function, $\tilde{f}^{0}(\boldsymbol{r}, \boldsymbol{v}, t)$ has the same expression as $f^{0}(\boldsymbol{r}, \boldsymbol{v}, t)$ if $\boldsymbol{u}$ is replaced by $\boldsymbol{u}+\frac{\boldsymbol{F}}{m} \tau$, and hence also in the discrete case. Integrating the above equation over a time interval $\triangle t$,

$$
\begin{gathered}
\text { L.H.S }=\int_{t}^{t+\Delta t} \frac{D}{D t} f_{i} d t=f_{i}\left(\boldsymbol{r}+\boldsymbol{v}_{i} \Delta t, t+\Delta t\right)-f_{i}(\boldsymbol{r}, t) \\
R . H . S=\int_{t}^{t+\Delta t}-\frac{f_{i}-\tilde{f}_{i}^{0}}{\tau}=-\frac{1}{\tau} \int_{t}^{t+\Delta t}\left\{f_{i}\left(\boldsymbol{r}+\boldsymbol{v}_{i} s, t+s\right)-\tilde{f}^{0}\left(\boldsymbol{r}+\boldsymbol{v}_{i} s, t+s\right)\right\} d s \\
\approx-\frac{1}{\tau} \frac{\Delta t}{2}\left\{f_{i}\left(\boldsymbol{r}+\boldsymbol{v}_{i} \triangle t, t+\triangle t\right)-\tilde{f}_{i}^{0}\left(\boldsymbol{r}+\boldsymbol{v}_{i} \Delta t, t+\triangle t\right)+f_{i}(\boldsymbol{r}, t)-\tilde{f}_{i}^{0}(\boldsymbol{r}, t)\right\}
\end{gathered}
$$

Following (Dellar,2001) this implicit scheme is made explicit by a change of variables. Let us use the modified distribution function

$$
\bar{f}_{i}(\boldsymbol{r}, t)=f_{i}(\boldsymbol{r}, t)+\frac{\triangle t}{2 \tau}\left\{f_{i}(\boldsymbol{r}, t)-f_{i}^{0}(\boldsymbol{r}, t)\right\} .
$$


Since, $f_{i}^{0}=\bar{f}_{i}^{0}$ and $\sum_{i} \bar{f}_{i}=\sum_{i} f_{i}=n$, where $n$ is the particle density, along with

$$
\begin{aligned}
\sum_{i} \boldsymbol{v}_{i} \bar{f}_{i} & =\sum_{i} \boldsymbol{v}_{i} f_{i}+\frac{\triangle t}{2 \tau}\left[\sum_{i} \boldsymbol{v}_{i} f_{i}-\sum_{i} \boldsymbol{v}_{i} \tilde{f}_{i}^{0}\right] \\
& =n \boldsymbol{u}+\frac{\triangle t}{2 \tau}\left[n \boldsymbol{u}-n\left(\boldsymbol{u}+\frac{\boldsymbol{F}}{m} \tau\right)\right] \\
& =n \boldsymbol{u}-n \frac{\triangle t}{2} \frac{\boldsymbol{F}}{m}
\end{aligned}
$$

We finally arrive at the following algorithm for the integration of the velocity field:

$$
\begin{array}{r}
\bar{f}_{i}\left(\boldsymbol{r}+\boldsymbol{v}_{i} \triangle t, t+\triangle t\right)-\bar{f}_{i}(\boldsymbol{r}, t)=-\frac{\Delta t}{\tau+\frac{\Delta t}{2}}\left[\bar{f}_{i}(\boldsymbol{r}, t)-\bar{f}_{i}^{0}(\boldsymbol{r}, t)\right], i=0 \ldots ., 18 \\
\frac{1}{n} \bar{f}_{i}^{0}=w_{i}\left[1+3 \frac{\boldsymbol{v}_{i} \tilde{\boldsymbol{u}}}{c^{2}}-\frac{3}{2}\left(\frac{\tilde{u}}{c}\right)^{2}+\frac{9}{2}\left(\frac{\boldsymbol{v}_{i} \tilde{\boldsymbol{u}}}{c^{2}}\right)^{2}\right] \\
n=\sum_{i} \bar{f}_{i} \\
\boldsymbol{u}=\frac{1}{n} \sum_{i} \boldsymbol{v}_{i} \bar{f}_{i}+\frac{1}{2} \triangle t \frac{\boldsymbol{F}}{m}
\end{array}
$$

with, $\tilde{\boldsymbol{u}}=\boldsymbol{u}+\tau \frac{\boldsymbol{F}}{m}$. On a macroscopic scale $n$ is known as fluid density and $\boldsymbol{u}$ is known as bulk velocity of the fluid. The sound velocity $c$ is fixed by $c=\frac{\Delta x}{\Delta t}$, where $\triangle x$ and $\triangle t$ are the mesh size and the time step, respectively. Relaxation time is fixed by $\tau=3 \frac{\nu}{c^{2}}$, where $\nu$ is the kinematic viscosity of the fluid.

\subsubsection{Stability and Accuracy}

The method is Mach number limited, in other words it is stable for low Mach number,

$$
M a=\frac{|\boldsymbol{u}|}{c}<0.115
$$

according to Lallemand \& Luo (2003).

The second and relatively unusual limit is that the time step should be small enough compared to the relaxation time

$$
\begin{aligned}
\frac{\tau+\frac{\Delta t}{2}}{\Delta t}>\frac{1}{1.93} & \\
& \Rightarrow \frac{\tau}{\triangle t}>\frac{1}{1.93}-\frac{1}{2} \Rightarrow \frac{1}{3} \frac{\tau}{\triangle t}>\frac{1}{3}\left(\frac{1}{1.93}-\frac{1}{2}\right) \\
& \Rightarrow \frac{\nu \triangle t}{\triangle x^{2}}>6.04 \times 10^{-3}
\end{aligned}
$$

The maximum Reynolds number on the grid scale can be derived from (3.21) and (3.22)

$$
R e_{\text {grid }}=\frac{u \triangle x}{\nu}=\frac{u}{c} \frac{\triangle x^{2}}{\nu \triangle t}<0.115 * 6 * 10^{-3}
$$


Accuracy requires that the truncation in Knudsen Number should be tolerable. For $10 \%$ accuracy, it is necessary that (Dellar 2001)

$$
0.1 \geq \frac{M a}{R e}
$$

which means

$$
\frac{M a}{R e}=\frac{u}{c} \frac{\nu}{u L}=\frac{u}{c} \frac{\frac{1}{3} c^{2} \tau}{u L}=\frac{1}{3} \frac{c \tau}{L}=\frac{1}{3} \frac{\lambda}{L} .
$$

Since $\lambda$ represents the mean-free path and $L$ represents the characteristic scale length, the above equation suggests their ratio should be less than or equal to $\frac{1}{30}$.

\subsubsection{Boundary Condition}

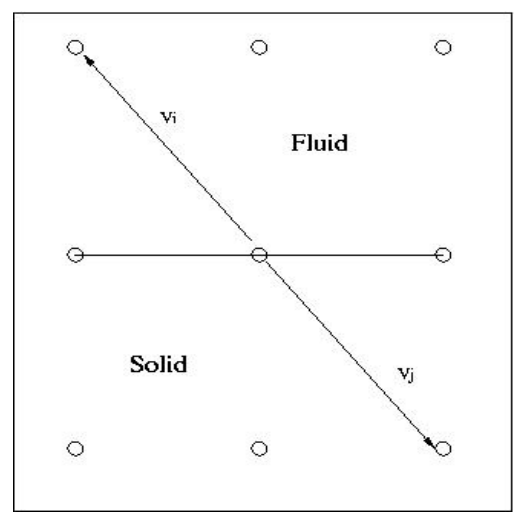

Figure 3.2: A pictorial view of the bounce back rule, where $\boldsymbol{v}_{\boldsymbol{i}}=-\boldsymbol{v}_{\boldsymbol{j}}$; small circles denote the nodal points

No-slip boundary conditions can be realized by a 'bounce back' scheme: a particle colliding with the wall reverses its momentum normal to the wall and maintains its tangential momentum unchanged. It is well understood now that the bounce-back scheme can achieve second order accuracy (Ginzbourg,d'Humières; 1996) if one is careful about the location of the boundary. The ease of handling boundary conditions with complicated geometries is an important feature of the LBM.

No-Slip Boundary: Fixed In general at no-slip fixed boundaries the vertical and horizontal components of the fluid are equal to zero, that means $\boldsymbol{u}=0$ all together:

If $\boldsymbol{u}=0 \Rightarrow \sum_{i} f_{i} \boldsymbol{v}_{i}=0$ at the boundary.

A convenient way to fulfill this criterion is to use the 'Bounce Back' rule. The rule is again adopted from the theory of Lattice Gas Automata. According to the rule, if moving particles face the boundary and at that time if $\boldsymbol{v}_{i}=-\boldsymbol{v}_{j}$ as in the figure (3.2), $f_{i}$ has to be equal to $f_{j}$ and in that case the $\boldsymbol{u}=0$ condition is maintained. This has to happen over a time step. Physically this implies the wall has to lie halfway between two nodal points, 


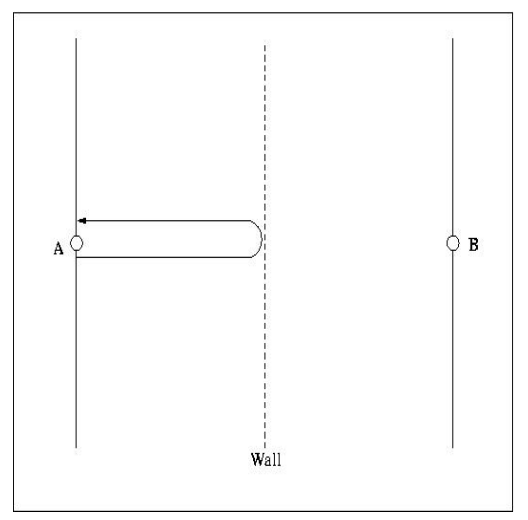

Figure 3.3: A is the fluid node and B is the solid node. The wall is situated exactly in between two nodes. The particle bounces back to A, but it covers the same distance as it would do while going from A to B.

one on solid and one on the fluid side. Particles with binary velocities are restricted to move the distance between two neighboring grid points over one time step. While following the bounce back rule this kind of movement is only maintained if the wall is situated exactly in-between two grid points as in fig. 3.3. Otherwise one needs to use an interpolation scheme. Following the bounce back rule one can write

$$
\bar{f}_{j}(t+\triangle t)=\bar{f}_{i}(t)-\frac{\triangle t}{\tau+\frac{\triangle t}{2}}\left(\bar{f}_{i}(t)-\bar{f}_{i}^{0}(t)\right)
$$

No-slip: Moving Even for a moving boundary, the no-slip condition should be satisfied, to incorporate realistic situations. In order to have such situation we set $\boldsymbol{v}_{i}=-\boldsymbol{v}_{j}$ and now $f_{i}$ and $f_{j}$ will hold some special relation to give the full form of the moving no-slip boundary (A.J.C.Ladd;1974):

$$
f_{j}(t+\triangle t)=\bar{f}_{i}(t)-6 w_{i} n \frac{\boldsymbol{v}_{i}}{c} \frac{\tilde{\boldsymbol{u}}_{b}}{c}-\frac{\triangle t}{\tau+\frac{\triangle t}{2}}\left(\bar{f}_{i}(t)-\bar{f}_{i}^{0}(t)\right)
$$

where, $\tilde{\boldsymbol{u}}_{b}=\boldsymbol{u}_{b}+\frac{\boldsymbol{F}}{m} \tau \boldsymbol{u}_{b}$ is the velocity of the wall and $w_{i}$ is the weight which occurs in $\bar{f}_{i}^{0}$.

\subsection{Spectral Method}

The spectral method is very popular in the field of hydrodynamics and magnetohydrodynamics. For dynamo simulations its use is very common in view of its high accuracy. 


\subsubsection{The Spectral Method to Solve Navier Stokes Equation}

The Navier Stokes equations for incompressible fluid flow may be written as

$$
\begin{array}{r}
\frac{\partial \boldsymbol{u}(\boldsymbol{r}, t)}{\partial t}+\boldsymbol{u}(\boldsymbol{r}, t) \cdot \nabla \boldsymbol{u}(\boldsymbol{r}, t)=-\nabla p(\boldsymbol{r}, t)+\frac{1}{R e} \nabla^{2} \boldsymbol{v}(\boldsymbol{r}, t), \\
\nabla \cdot \boldsymbol{u}(\boldsymbol{r}, t)=0,
\end{array}
$$

where $\boldsymbol{u}(\boldsymbol{r}, t)$ the velocity, $p(\boldsymbol{r}, t)$ pressure and $R e$ the hydrodynamic Reynolds number. To implement a periodic boundary condition in a cubical box of length $L$ we assume

$$
\begin{aligned}
& \boldsymbol{u}\left(\boldsymbol{r}+\frac{2 \pi}{L} \boldsymbol{n}, t\right)=\boldsymbol{u}(\boldsymbol{r}, t) \\
& \text { where, } \boldsymbol{n}=\left(n_{1}, n_{2}, n_{3}\right), n_{i}=0, \pm 1, \pm 2, \ldots
\end{aligned}
$$

With the above assumption of periodicity the velocity field can be written as

$$
\boldsymbol{u}(\boldsymbol{r}, t)=\sum_{\boldsymbol{k}} \boldsymbol{u}_{\boldsymbol{k}} \exp (i \boldsymbol{k} \cdot \boldsymbol{r})
$$

where, $\boldsymbol{k}=\left(\frac{2 \pi}{L}\right) \boldsymbol{n}$ and the pressure as

$$
p(\boldsymbol{r}, t)=\sum_{\boldsymbol{k}} p_{\boldsymbol{k}} \exp (i \boldsymbol{k} \cdot \boldsymbol{r})
$$

Using (3.28) \& (3.29) in (3.27a) \& (3.27b) one can write the Navier Stokes in the spectral space

$$
\begin{array}{r}
\left(\frac{d}{d t}+\frac{1}{R e} \boldsymbol{k}^{2}\right) \hat{\boldsymbol{u}}_{\boldsymbol{k}}=-i \boldsymbol{k} \hat{p}_{\boldsymbol{k}}-(\widehat{\boldsymbol{u} \cdot \nabla \boldsymbol{u}})_{\boldsymbol{k}} \\
i \boldsymbol{k} \cdot \hat{\boldsymbol{u}}_{\boldsymbol{k}}=0
\end{array}
$$

Let us consider, $\hat{\boldsymbol{f}}_{\boldsymbol{k}}=-(\widehat{\boldsymbol{u} \cdot \nabla \boldsymbol{u}})_{\boldsymbol{k}}$. This non-linear term is mostly responsible for the complexity of the problem. Now taking $i k$ dotted into (3.30a) and using (3.30b) we get

$$
\begin{gathered}
\hat{p}_{\boldsymbol{k}}=-\frac{1}{|\boldsymbol{k}|^{2}} i \boldsymbol{k} \cdot \hat{\boldsymbol{f}}_{\boldsymbol{k}}, \\
\left(\frac{d}{d t}+\frac{1}{R e} \boldsymbol{k}^{2}\right) \hat{\boldsymbol{u}}_{\boldsymbol{k}}=\hat{\boldsymbol{f}}_{\boldsymbol{k}}-\frac{\boldsymbol{k}\left(\boldsymbol{k} \cdot \hat{\boldsymbol{f}}_{\boldsymbol{k}}\right)}{|\boldsymbol{k}|} .
\end{gathered}
$$

Now the Adams-Bashforth method is employed for time differencing of the nonlinear term $\hat{\boldsymbol{f}}_{\boldsymbol{k}}$ and Crank-Nicolson for the viscous term. If $t_{n}$ denotes the time at which the unknowns are computed, then the time marching scheme can be represented as

$$
\hat{\boldsymbol{u}}_{\boldsymbol{k}}\left(t_{n+1}\right)=\frac{\left(1-\frac{h}{2} \frac{|\boldsymbol{k}|^{2}}{R e}\right) \hat{\boldsymbol{u}}_{\boldsymbol{k}}\left(t_{n}\right)+i \boldsymbol{k} \frac{h}{2}\left\{-3 \hat{\boldsymbol{f}}_{\boldsymbol{k}}\left(t_{n}\right)+\hat{\boldsymbol{f}}_{\boldsymbol{k}}\left(t_{n-1}\right)\right\}}{1+\frac{h}{2} \frac{|\boldsymbol{k}|^{2}}{R e}} .
$$

In case of external forcing, the forcing term is also implicit inside $\hat{\boldsymbol{f}}_{\boldsymbol{k}}$. 


\subsubsection{The Spectral Method to Solve the Induction Equation}

The dimensionless induction equation in real space can be written as

$$
\partial_{t} \boldsymbol{B}+\nabla \times(\boldsymbol{B} \times \boldsymbol{u})=\frac{1}{R_{m}} \nabla^{2} \boldsymbol{B}, \nabla \cdot \boldsymbol{B}=0
$$

where $R_{m}$ is a dimensionless number, known as magnetic Reynolds number. The solution is obtained in the same manner as in the Navier-Stokes equation. The Fourier decomposition for the magnetic field $\boldsymbol{B}$ is

$$
\boldsymbol{B}(\boldsymbol{r}, t)=\sum_{k} \hat{\boldsymbol{B}}_{\boldsymbol{k}}(t) \exp (i \boldsymbol{k} \cdot \boldsymbol{r})
$$

where the sum is over all wave numbers, and $\boldsymbol{k}$ is compatible with the boundary conditions. From here follows the induction equation in spectral space:

$$
\frac{d}{d t} \hat{\boldsymbol{B}}_{\boldsymbol{k}}+i \boldsymbol{k} \times(\widehat{\boldsymbol{B} \times \boldsymbol{u}})_{\boldsymbol{k}}=\frac{1}{R_{m}}|\boldsymbol{k}|^{2} \hat{\boldsymbol{B}}_{\boldsymbol{k}}
$$

For time marching again a second-order Adams-Bashforth scheme is used for the $\boldsymbol{B} \times \boldsymbol{u}$ term coupled to a Crank-Nicolson scheme for the diffusion term. If $t_{n}$ denotes the times at which the unknowns are computed, the time marching scheme is in the formula:

$$
\begin{aligned}
\left(1+\frac{h}{2} \frac{|\boldsymbol{k}|^{2}}{R_{m}}\right) \hat{\boldsymbol{B}}_{\boldsymbol{k}}\left(t_{n+1}\right)=\left(1-\frac{h}{2} \frac{|\boldsymbol{k}|^{2}}{R_{m}}\right) \hat{\boldsymbol{B}}_{\boldsymbol{k}}\left(t_{n}\right)+i \boldsymbol{k} \frac{h}{2}\left(-3(\widehat{\boldsymbol{B} \times \boldsymbol{u}})_{\boldsymbol{k}}\right)\left(t_{n}\right)+ & \left.(\widehat{\boldsymbol{B} \times \boldsymbol{u}})_{\boldsymbol{k}}\left(t_{n-1}\right)\right) .
\end{aligned}
$$

\subsection{Advantages and Disadvantages of LBM and Spectral Method}

Every numerical scheme has its own advantages and disadvantages. The method to be chosen depends on the physical problems encountered. In this thesis the main physical aim is to simulate the Karlsruhe dynamo. The spectral method is so far an established method to solve dynamo problems for its accuracy. It is a fast algorithm as well. Several geodynamo models and astrophysical dynamo models are based on the spectral method. But in all these cases the geometry was simple enough: either a simple spherical or a cylindrical domain was enough for this kind of simulation. On the other hand, the Karlsruhe dynamo is constrained by several complex geometries, the guided pipes and blades having made the situation difficult for simulation. So far several simulations exist (eg. Tilgner,1997;Tilgner,2002), neglecting these complexities.

But simulation including complex walls are also important and cannot be ignored. Hence, a proper numerical scheme needs to be evolved. LBM is quite convenient for this situation, the method itself is easy to implement. So far the method was mainly famous because of its capability to handle complex walls. Definitely this is a second order accurate method for the solution of the induction equation which may bring up artefacts. So here mainly LBM is used to simulate the Navier-Stokes equation, and the spectral method 
is used to simulate the induction equation. They are coupled together so that the output of one can go smoothly with the other. In the next chapter the methods of coupling will be discussed in detail. 



\section{Numerical Experiments and code coupling}

\subsection{Introduction}

In this chapter we describe mainly the way we have developed the lattice-Boltzmann code, which ultimately solves the 3-dimensional Navier-Stokes equation. When describing its development we will speak about the various tests we went through. Some of them with linear solutions will be verified analytically. The non-linear tests are verified with established results.

Once we complete the Navier-Stokes solver development we want to couple that with the spectral code, which solves the induction equation. The coupling is described in the second half of this chapter.

\subsection{Numerical Experiments}

\subsubsection{Flow through a channel}

The initial simulation is done for a simple laminar flow through a channel. This is a two dimensional problem. The simulation is done in a square box of unite length. Particularly this problem is chosen because there exists an analytical solution. We define a Cartesian co-ordinate system. The $x$ axis is along the horizontal direction of the box and $y$ axis is along its vertical direction. An arrangement of this kind is shown in figure (4.1). The fluid is driven by a constant force field, which acts in $x$ direction. The upper and lower ends of the box is bounded by no-slip walls.

In general, the dimensionless equation of motion can be written as

$$
\frac{\partial \boldsymbol{v}}{\partial t}+(\boldsymbol{v} \cdot \nabla) \boldsymbol{v}=-\nabla p+\frac{1}{R_{e}} \nabla^{2} \boldsymbol{v}+\boldsymbol{f}
$$

where $\boldsymbol{v}$ is the velocity of the fluid, $p$ is the pressure, $f$ is the applied force to drive the flow and $R_{e}$ is the Reynolds number of the flow. Now in the steady state $\frac{\partial \boldsymbol{v}}{\partial t}=0$; the $(\boldsymbol{v} \cdot \nabla) \boldsymbol{v}$ term is also zero for the here considered flow along $x$ direction. Hence, if we neglect the pressure gradient force (pressure is constant throughout the channel), the 


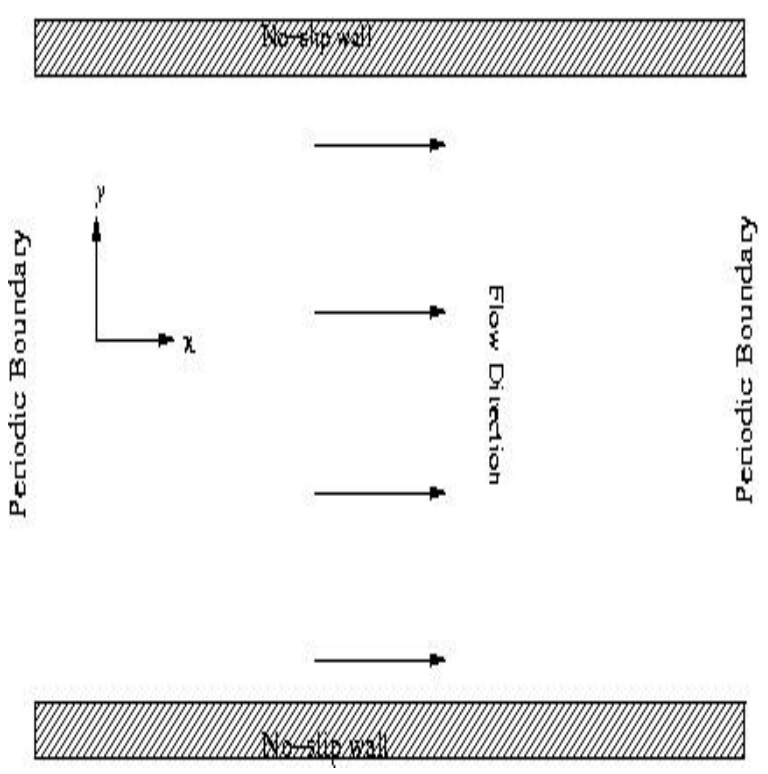

Figure 4.1: Flow through a channel:Schema of the simulation arrangement

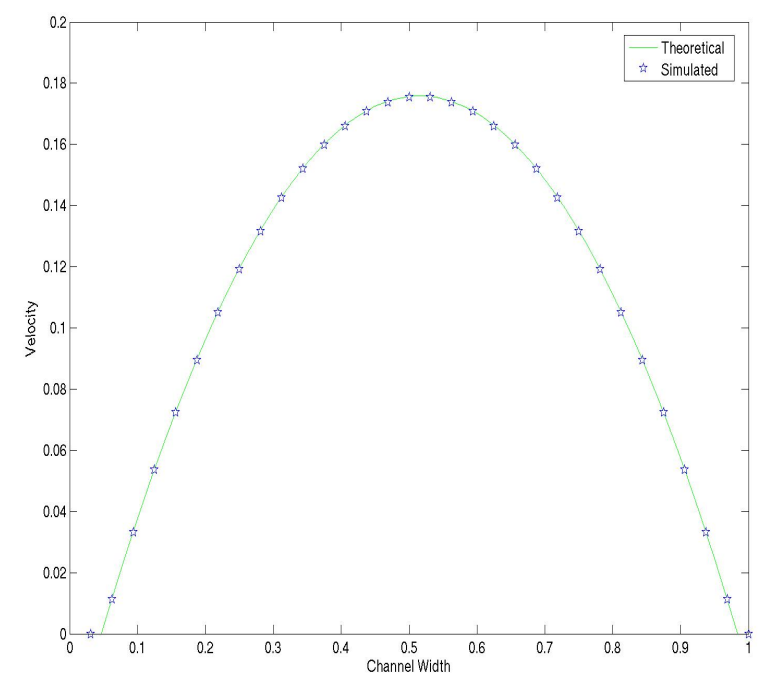

Figure 4.2: Flow through a channel: the simulation result is different from the theoretical one near to the wall, this is just because the wall lies between two nodal points

equation (4.1) turns into

$$
0=\frac{1}{R_{e}} \nabla^{2} \boldsymbol{v}+\boldsymbol{f}
$$

Assuming the $x$ component of the velocity $\boldsymbol{v}_{x}$ is driven by the $x$ component of the force $f_{x}$ we have

$$
0=\frac{1}{R_{e}} \frac{d^{2} v_{x}}{d y^{2}}+f_{x}
$$


After integrating the above equation we get

$$
v_{x}=-\frac{R_{e} f_{x}}{2} y^{2}+C_{1} y+C_{2}
$$

where $C_{1}$ and $C_{2}$ are constants, which are to be determined using boundary conditions. Now since the boundary wall is no-slip the velocity of the fluid must be zero at the wall. To be compatible with the numerical simulation we take the boundary condition as

$$
\begin{aligned}
& v_{x}=0 \text { at } y=0.046875, \\
& v_{x}=0 \text { at } y=0.984375,
\end{aligned}
$$

and after taking $f_{x}=0.08, R_{e}=20$ we obtain $C_{1}=0.8245$ and $C_{2}=-0.0366$.

So the overall velocity profile across the box becomes

$$
v_{x}=-0.8 y^{2}+0.8245 y-0.0366 .
$$

The simulation has been run for the time and spatial resolution $d t=0.001 d x=d y=\frac{1}{32}$, which keeps the Mach number within 0.1. This is to be followed while the Lattice Boltzmann Method (LBM) simulation is carried out. It has been run until the kinematic energy became saturated. In figure (4.2) the simulation result for the middle of the box at $x=0.5$ is plotted against the analytical solution. The simulation result agrees with the theoretical prediction within the error limit of LBM (second order). Near the wall the discrepancy is due to the fact that the wall lies in-between two grid points.

\subsubsection{Flow past a circlular object}

Flow past a circular object is a very common numerical experiment, and is done using various numerical methods. The basic equation is same as equation (4.1). As a test case we use it in order to have a wide choice of published results for comparison. At high Reynolds number (of, say, $R_{e}=1000$ ) any flow becomes turbulent, but the presence of obstacles makes it turbulent at lower Reynolds number. At what $R_{e}$ the flow will become turbulent, of course, depends on the size of the obstacle and the size of the channel through which the fluid is flowing.

For our simulation we have used a rectangular box of width 2 and length 5, a circular object of radius 0.25 placed at co-ordinate $(1.00,1.05)$ (assuming the origin at the lower left corner of the box). The box is periodic along the length i.e. left and right sides of the box have periodic boundaries, whereas the top and bottom walls are no-slip.

It is seen in this kind of flow at very low $R_{e}$, the flow divides ahead of the obstacle only to reunite immediately behind it, and the flow becomes steady after a certain time. Above $R e=60$ the flow loses the symmetry and above $R_{e}=130$ the flow becomes turbulent and starts producing a Kármán vortex street.

In our simulation the spatial resolution has been taken $d x=d y=0.1$ and the temporal resolution $d t=0.005$. We start the simulation with zero velocity, over which a constant force-field along the horizontal direction $\left(f_{x}=0.08\right)$ is applied throughout the box. In 

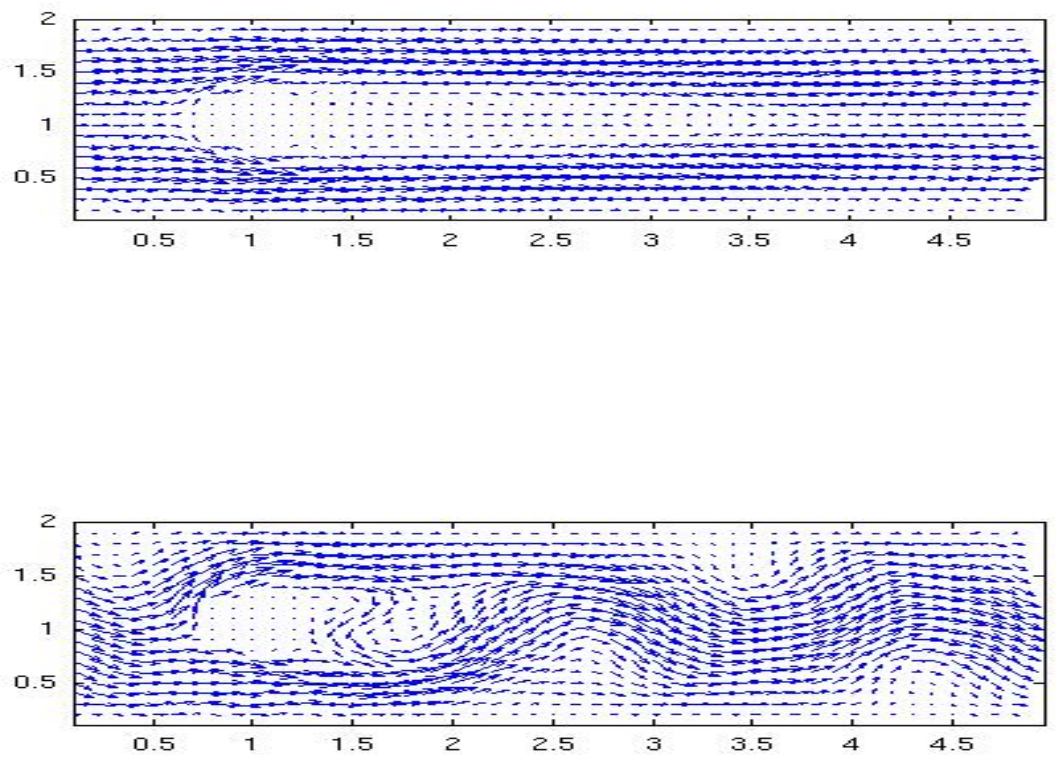

Figure 4.3: Flow past a circular object : Two figures show different time snapshots of the velocity field. Initially the flow looks like the top figure; with time it becomes turbulent and takes the shape of the bottom figure. The circular object is centered at $(1,1.05)$ with radius 0.25 . The simulation is done for $R_{e}=140$.

the figure (4.3) two snapshots of the simulation are presented. The arrows indicate the velocity vector. Initially the flow is laminar because of the low velocity, but later with increasing velocity the flow shows a turbulent nature. This simulation has been carried out with $R_{e}=140$.

\subsubsection{Taylor-Couette Flow}

The Taylor-Couette (TC) experiment was one of the famous hydrodynamic experiments in the last century. The basic arrangement was to see the fluid instability in the space between two coaxial rotating cylinders and its main purpose was to investigate a potentially unstable arrangement of flow resulting from a prevailing adverse gradient of angular momentum. The experiment is well documented and later there were numerical simulations as well to reproduce the original experiment. Recently also the magneto-hydrodynamic effect on the TC experiment has been studied (Rüdiger,Shalybkov, 2001). In this context the TC-experiment is particularly appropriate to verify the applicability of our 3D code. The implementation of the rotating cylindrical wall is also a crucial point of our numerical simulation. 
In our simulation we consider the case when the outer cylinder is fixed and the inner cylinder rotating, i.e. angular velocity of the fluid (situated between the two concentric cylinders) decreases outwards with the radius. Assuming that the radius of the inner cylinder is $r_{i}$ and the radius of the outer cylinder $r_{0}=1$, the dimensionless velocity of the inner cylinder is

$$
\boldsymbol{v}_{i}\left(r_{i}\right)=\hat{\boldsymbol{\phi}}
$$

where $\hat{\phi}$ is the unit vector in the azimuthal direction. The dimensionless velocity of the outer cylinder having unit radius is

$$
\boldsymbol{v}_{0}\left(\boldsymbol{r}_{0}=1\right)=0 .
$$

The horizontal cut of the simulation box is shown in figure (4.5). Now from the definition of the Taylor number we get

$$
T_{a}=\frac{\Omega^{2} r_{i} a^{3}}{\nu^{2}}
$$

where $\Omega$ is the rotational velocity of the inner cylinder, $\nu$ is the kinematic viscosity of the fluid, $a$ is the distance between two cylinders. We can then write down, as per our notation,

$$
T_{a}=R_{e}^{2} \frac{r_{0}}{r_{i}}\left(1-\frac{r_{i}}{r_{0}}\right)^{3} .
$$

Here $R_{e}=\Omega r_{i}^{2} \nu$ is the hydrodynamic Reynolds number. With the aid of equation (4.6) we can express the Taylor number $\left(T_{a}\right)$ in terms of the hydrodynamic Reynolds number, once the inner and outer cylinder radii are known. Hence the critical Taylor number $\left(T_{a_{c}}\right)$ also corresponds to critical hydrodynamic Reynolds number $\left(R_{e_{c}}\right)$.

Above the critical number, flow becomes unstable and shows Taylor instability. Initially, the flow is axisymmetric; but for high $T_{a}$, it loses the axisymmetric pattern and eventually becomes turbulent. The Taylor-instability pattern is characterized by its wavelength. While the flow is unstable, it looks like figure (4.4) which shows the meridional cut through the cylinders. Even though the inner-cylinder is rotating in $\phi$ direction, flow gets a vertical component when it is unstable (above critical $T_{a}$ or $R_{e}$ numbers), which forms a toroidal pattern around the cylindrical axis. This pattern is periodic along $z$ direction. The height of each cell is $H=\pi\left(r_{0}-r_{i}\right) / 3.1$. The schemata of these cells are shown in figure (4.4).

In our simulation we do the experiment within a box of length and width 2.2 and height 1.2. This fits two cylinders well. $r_{0}=1$ demands a box of length and width 2 , the excess dimension is due to the wall thickness. The height of the box can hold at least two periodic cells of the instability pattern. The simulation is periodic in horizontal and vertical direction. In this simulation we take $r_{i}=0.5$, the spatial resolution is $d x=d y=0.034$ and the time resolution is $d t=0.0015$, we take $R_{e}=155$ which is well above the $R_{e_{c}}$. The $z$ component of the velocity field starts growing from zero and shows a periodic cell-like pattern (figure (4.6)), which eventually becomes stationary with time. The height of each cell agrees with the previously mentioned cell height 

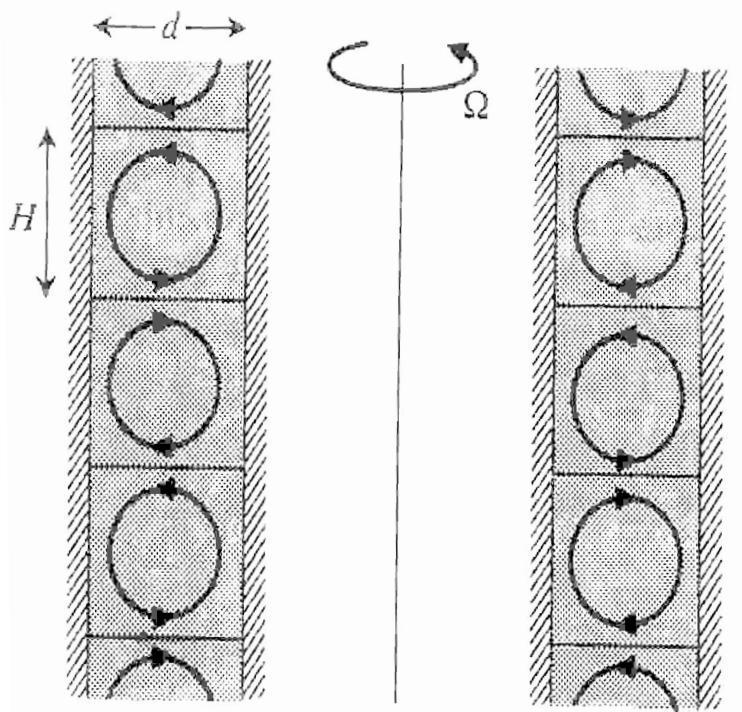

Figure 4.4: Schema of the meridional cut through the cylinders show the instability cells of height $H$ when the inner cylinder is rotating with angular velocity $\Omega$ and the outer cylinder is static.

$$
H=\frac{\pi(1-0.5)}{3.1}=0.508
$$

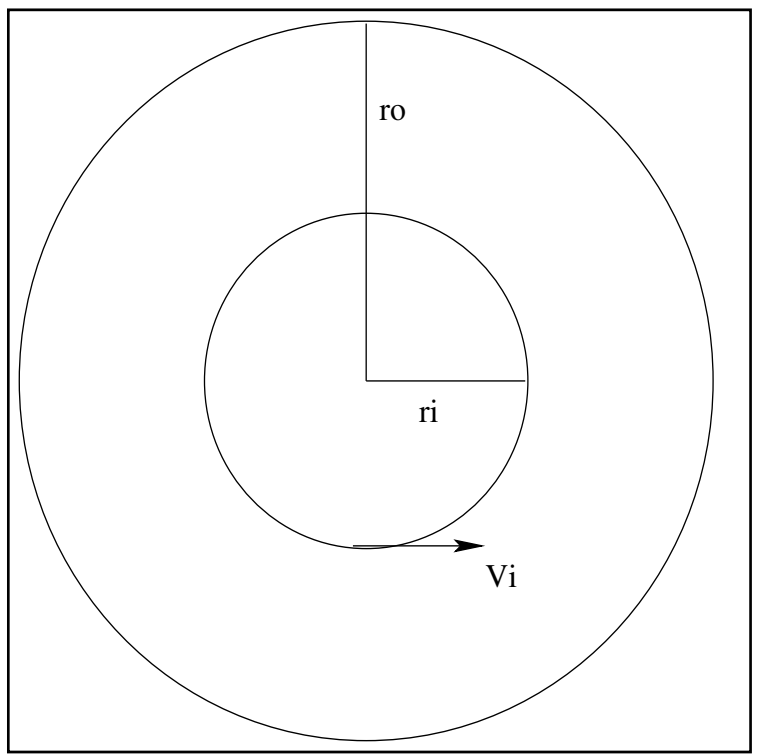

Figure 4.5: A horizontal cross section of the simulation box, showing two co-axial cylinders having radii $r_{i} \& r_{0}$, the inner cylinder rotates with velocity $\boldsymbol{v}_{i}$. 


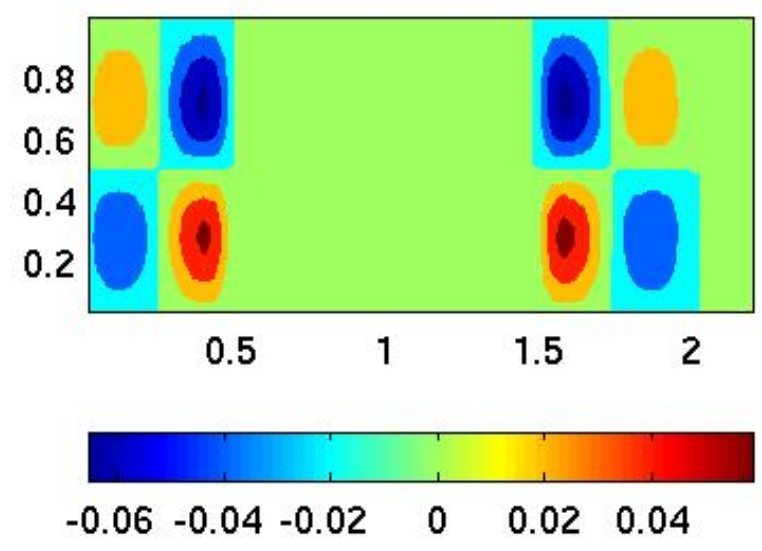

Figure 4.6: A meridional cut of the original simulation: color contour shows the $z$ component of velocity, in a box of length 1 there are two cells when $r_{0}-r_{i}=0.5$, which agrees with the theoretical prediction as well as with the experiment.

\subsection{Coupling of Spectral and LBM codes}

Both the design and data analysis of the laboratory dynamo experiments require heavy numerical computation (Tilgner, 1997; Tilgner, 2002). These simulations are complicated by the fact that unlike the real Earth, the experiment always contains some mechanical structure of complex geometry. That is why flow simulations accompanying such experiments are best done with a flexible method like the LBM. Previous dynamo simulations have mostly used spectral methods. There are three reasons why spectral methods are favored when computing magnetic fields. First, the boundary conditions at a conductor/insulator interface are nonlocal. They acquire simple expressions when the magnetic field is spectrally decomposed in a suitable base function provided that the fluid is confined to plane layers, infinite cylinders, or spheres. Second, the condition that there be no magnetic monopoles can be implemented, particularly accurately in a spectral method. Only recently was an LBM able to keep the magnetic field solenoidal down to roundoff errors in a magnetohydrodynamic simulation (Dellar,2002; Breyiannis, Valougeorgis, 2004). Related to this point is the third reason in favor of spectral methods, which is their convergence properties. When dealing with dynamos one is first faced with a binary question: is a given flow capable of generating a magnetic field, yes or no? Because of this dividing line between dynamos and non-dynamos, small numerical errors can lead to qualitatively wrong results, e.g. a zero magnetic field even though the flow is capable of dynamo action or vice versa. In order to prevent catastrophes of this type, it is better to use an accurate high order scheme to simulate the magnetic field.

The experiments which are running or being planned at the moment have outer boundaries which can be reasonably approximated by one of the geometries amenable to spec- 
tral methods. Mechanical structures inside the experimental cell, which serve to drive or guide the flow of the liquid conductor, are made of material of similar electrical conductivity as the fluid. They thus constrain the flow but not the electrical currents. We are, therefore, prompted to develop methods which use spectral methods for the equations of electrodynamics and the LBM for solving the Navier-Stokes equation. We demonstrate this coupling strategy here for a case in which fully spectral method can also be used for validation (Sarkar, Tilgner;2005).

In the present case it is convenient to use identical grids for the LBM and the spectral method. This is not possible when using adaptive grids or Non-Cartesian geometries. It will then be necessary to interpolate the velocity field computed by the LBM onto the grid of the spectral method. Since the typical LBM yields second order accurate results, a quadratic interpolation will suffice to maintain the overall order of the method. An interpolation step, therefore, should not be a major obstacle when coupling LBM and spectral methods for other problems than the one treated here.

We consider the model (G. O. Roberts,1971) which has inspired the so called "Karlsruhe dynamo experiment" as explained before in equation (2.35). An infinite expanse of liquid conductor is set into motion so that the velocity field consists of a periodic array of right handed helices. A box with periodic boundary conditions has the appropriate geometry to deal with this problem.

We solve here the coupled non-dimensional equations

$$
\begin{array}{r}
\frac{\partial \boldsymbol{u}}{\partial t}+\boldsymbol{u} \cdot \nabla \boldsymbol{u}=-\nabla p+\frac{1}{R_{e}} \nabla^{2} \boldsymbol{u}+(\nabla \times \boldsymbol{B}) \times \boldsymbol{B}+\boldsymbol{f} \\
\nabla \cdot \boldsymbol{u}=0 \\
\frac{\partial \boldsymbol{B}}{\partial t}=\nabla \times(\boldsymbol{u} \times \boldsymbol{B})+\frac{1}{R_{m}} \nabla^{2} \boldsymbol{B} \\
\nabla \cdot \boldsymbol{B}=0,
\end{array}
$$

in which two control parameters appear: The Reynolds number $R_{e}$ and the magnetic Reynolds number $R_{m}$. Here $p$ is the pressure, $\boldsymbol{u}(\boldsymbol{r}, t)$ is the non-dimensional velocity and $\boldsymbol{B}(\boldsymbol{r}, t)$ is the non-dimensional magnetic field. $\boldsymbol{f}(\boldsymbol{r})$ is a forcing which maintains the flow against viscous dissipation and the magnetic force $(\nabla \times \boldsymbol{B}) \times \boldsymbol{B}$. $\boldsymbol{f}$ is chosen to be

$$
\boldsymbol{f}=\frac{2}{R_{e}}\left(\frac{2 \pi}{a}\right)^{2} \boldsymbol{u}_{0}
$$

with

$$
\boldsymbol{u}_{0}=\left(\begin{array}{c}
\sqrt{2} \sin \left(\frac{2 \pi}{a} x\right) \cos \left(\frac{2 \pi}{a} y\right) \\
-\sqrt{2} \cos \left(\frac{2 \pi}{a} x\right) \sin \left(\frac{2 \pi}{a} y\right) \\
2 \sin \left(\frac{2 \pi}{a} x\right) \sin \left(\frac{2 \pi}{a} y\right)
\end{array}\right) .
$$

The equations are solved subject to periodic boundary conditions in the domain $0 \leq x \leq$ $a, 0 \leq y \leq a, 0 \leq z \leq a$. As long as $\boldsymbol{B}=0, \boldsymbol{u}_{0}$ is a steady state solution of equation (4.7a). At large enough $R_{e}$, this solution becomes unsteady. Any magnetic field generated by the dynamo effect will also distort the velocity field. 
We give here a validation of the method presented in the previous section by comparing it to a purely spectral method in which Navier-Stokes is solved by Fourier decomposition, too. For an easy comparison, parameters are chosen so that a stationary state rather than a time dependent state is reached after a sufficiently long integration. The results are listed in table(4.1). All runs are for $R_{e}=1, R_{m}=30, a=1$ and they have been started from the initial conditions $\boldsymbol{u}(\boldsymbol{r}, t=0)=0$ and $\boldsymbol{B}(\boldsymbol{r}, t=0)=0.5 \hat{\boldsymbol{x}}$ where $\hat{\boldsymbol{x}}$ is the unit vector in $x$-direction. The time evolution has been integrated up to $\mathrm{t}=20$. Table 4.1 compares kinetic and magnetic energies, $E_{k i n}$ and $E_{B}$, defined by

$$
E_{k i n}=\int_{0}^{a} d x \int_{0}^{a} d y \int_{0}^{a} d z \boldsymbol{u}^{2} / 2 \text { and } E_{B}=\int_{0}^{a} d x \int_{0}^{a} d y \int_{0}^{a} d z \boldsymbol{B}^{2} / 2
$$

The energies are computed with the trapezoidal rule in all codes. For stability reasons, the time step had to be chosen smaller for the LBM than for the spectral method. At higher $R_{e}$, the stability limits for both methods become identical. The execution time per time step is larger for the hybrid code by roughly a factor of 2 . As expected, the results in table (4.1) become independent of spatial resolution already at lower resolution for the purely spectral method than for the hybrid code. This is a manifestation of the better convergence properties of the spectral method and of the fact that this particular problem is well suited for Fourier expansions. At large resolutions, both methods yield results in reasonable agreement which validates the hybrid code.

In the following chapter we use the above mentioned code to simulate the Karlsruhe dynamo; in some cases only the spectral code is also used in the simulation process, when the presence of complex boundaries is not critical.

\begin{tabular}{|r|r|r|r|r|}
\hline method & resolution & $\triangle t$ & $E_{\text {kin }}$ & $E_{B}$ \\
\hline \hline SPECTRAL & $8^{3}$ & $2 \times 10^{-3}$ & 0.7737 & 2.1568 \\
& & $1 \times 10^{-3}$ & 0.7737 & 2.1568 \\
\hline & $16^{3}$ & $2 \times 10^{-3}$ & 0.8482 & 1.4271 \\
& & $1 \times 10^{-3}$ & 0.8482 & 1.4271 \\
\hline & $32^{3}$ & $2 \times 10^{-3}$ & 0.8482 & 1.4268 \\
\hline LBM & $8^{3}$ & $1 \times 10^{-3}$ & 0.6805 & 1.586 \\
& & $2.5 \times 10^{-4}$ & 0.668 & 1.598 \\
\hline & $16^{3}$ & $2.5 \times 10^{-4}$ & 0.819 & 1.312 \\
\hline & $32^{3}$ & $1 \times 10^{-4}$ & 0.842 & 1.401 \\
\hline \hline
\end{tabular}

Table(4.1): Compares kinematic and magnetic energies of various time and spatial resolutions in spectral method and LBM runs 



\section{Numerical Simulations of the Karlsruhe Dynamo}

In order to simulate the Karlsruhe Dynamo we solve the previously mentioned coupled dimensionless equations (4.7). The translation between the non-dimensional variables and physical variables relating to the experiment involves a length scale $l_{s}$, a time scale $t_{s}$ and a magnetic field strength $B_{s} . l_{s}$ is fixed by identifying a length in the simulation with a length in the experiment, eg., the size of two periodic cells together, which leads to $l_{s} \approx 0.42 \mathrm{~m}$. In order to recover a dimensional induction equation from $(4.7 \mathrm{c})$ one has to choose for the time scale $t_{s}=\frac{l_{s}^{2}}{\lambda R_{m}}$, where $\lambda$ is the magnetic diffusivity of the fluid. (4.7a) is consistent with the dimensional Navier-Stokes equation for $B_{s}=\sqrt{\rho \mu_{0}} \lambda R_{m} / l_{s}$, where $\rho$ is the density of the fluid and $\mu_{0}$ the vacuum permeability. $B_{s}$ thus depends on $R_{m}$.

The values used for the magnetic Reynolds number $R_{m}$ in the simulation are realistic but the hydrodynamic Reynolds number $R_{e}$ is far smaller than any realistic estimate in the order of $10^{6}$. This is because of two reasons: (I) It is too expensive to computationally reach such a high value. (II) The flow becomes unstable when $R_{e}$ assumes such a high value and then bears little resemblance to the flow realized in the Karlsruhe Dynamo. In this chapter most of the quantities are dimensionless unless mentioned otherwise.

\subsection{Some previous calculations}

As mentioned earlier, Busse (1992) suggested the feasibility of this two-scale homogeneous dynamo. Busse's calculation and later numerical result of Tilgner (1997) showed that the detailed flow structure (involving for instance a boundary layer) of individual cylinder is not very important to produce a dynamo. Later Tilgner $(1997,2002)$ using the spectral method and Rädler et al. $(1998,2002 \mathrm{a})$ using the mean-field model showed that the non-symmetric mode with an azimuthal wave number $m=1$ shows the largest amplification for all magnetic Reynolds numbers. The mean magnetic field has a 'spiral staircase' structure in the near field and dipolar orientation perpendicular to the cylinder axis.

Later, the saturation mechanism of the dynamo also became of interest. A characteristic feature of the observed dynamo action is the measured significant increase of the pressure losses in the flow channels of the test module after the onset of self-excitation. The increase is caused by the occurrence of the Lorentz force which gives rise to an additional 
pressure. There were some numerical simulations (Tilgner 2001, Tilgner \& Busse 2002, Brandenburg \& Rädler 2003) considering the back-reaction of the Lorentz force on the fluid flow. Rädler et al. (2002b) showed the effect of the back-reaction using the meanfield theory.

It is important to see the effect of the Lorentz force on the overall flow structure, how the flow structure is affected by the Lorentz force when the magnetic field is strong enough. Tilgner \& Busse (2001) in their analytical calculation showed that for very low hydrodynamic Reynolds number (Re) (i.e. neglecting the inertial term ) and at the saturation of dynamo, Roberts velocity profile gets modified by the Lorentz force. Since the alpha effect is solely a functional of the flow field $\boldsymbol{u}_{\mathbf{0}}$, the modified velocity profile will modify the alpha effect, which will now be also a function of the mean magnetic field $\overline{\boldsymbol{B}}$. This phenomenon can be termed as 'alpha quenching'. It starts with the simple Roberts velocity profile $\boldsymbol{u}_{\mathbf{0}}$ and arrive finally (under above mentioned conditions) at a profile

$$
\boldsymbol{u}=(1-\gamma) \boldsymbol{u}_{\mathbf{0}}+2 \gamma \frac{\bar{B}_{x} \bar{B}_{y}}{{\overline{B_{x}}}^{2}+\bar{B}_{y}{ }^{2}} \tilde{\boldsymbol{u}}
$$

where

$$
\begin{gathered}
\boldsymbol{u}_{\mathbf{0}}=v_{0}\left(\begin{array}{c}
\sqrt{2} \cos \left(\frac{\pi x}{a}\right) \sin \left(\frac{\pi y}{a}\right) \\
\sqrt{2} \sin \left(\frac{\pi x}{a}\right) \cos \left(\frac{\pi y}{a}\right) \\
2 \cos \left(\frac{\pi x}{a}\right) \cos \left(\frac{\pi y}{a}\right)
\end{array}\right) \\
\tilde{\boldsymbol{u}}=v_{0}\left(\begin{array}{c}
\sqrt{2} \sin \left(\frac{\pi x}{a}\right) \cos \left(\frac{\pi y}{a}\right) \\
\sqrt{2} \cos \left(\frac{\pi x}{a}\right) \sin \left(\frac{\pi y}{a}\right) \\
2 \sin \left(\frac{\pi x}{a}\right) \sin \left(\frac{\pi y}{a}\right)
\end{array}\right) \\
\gamma=\frac{R_{e} R_{m}}{4}\left(\frac{a}{\pi}\right)^{2}\left(\bar{B}_{x}^{2}+\bar{B}_{y}^{2}\right)
\end{gathered}
$$

This calculation shows firstly that to sustain the amplitude of the original velocity distribution $\boldsymbol{u}_{0}$, the forcing must be increased by $1+\gamma+O\left(\gamma^{2}\right)$ and secondly that the original velocity field is modified by the effect alpha quenching, which is shown in figure (5.1).

They showed that if the modified velocity pattern is put into the induction equation the mean field evolution looks like

$$
\partial_{t} \overline{\boldsymbol{B}}+\nabla \times A\left(\begin{array}{c}
\bar{B}_{x} \\
\bar{B}_{y} \\
0
\end{array}\right)-\nabla \times C \bar{B}_{x} \bar{B}_{y}\left(\begin{array}{c}
\bar{B}_{y} \\
\bar{B}_{x} \\
0
\end{array}\right)=\frac{1}{R_{m}} \nabla^{2} \overline{\boldsymbol{B}}
$$

with

$$
A=v_{0}^{2} \frac{a}{\sqrt{2} \pi} R_{m}, C=A\left(\frac{a}{\pi}\right)^{2} \frac{R_{m} R_{e}}{2} .
$$

\subsection{An analytical derivation of the wave}

Equation (5.1) supports waves if an external uniform magnetic field is applied. These waves are magnetic in nature but different from the Alfvén waves, because to arrive at 


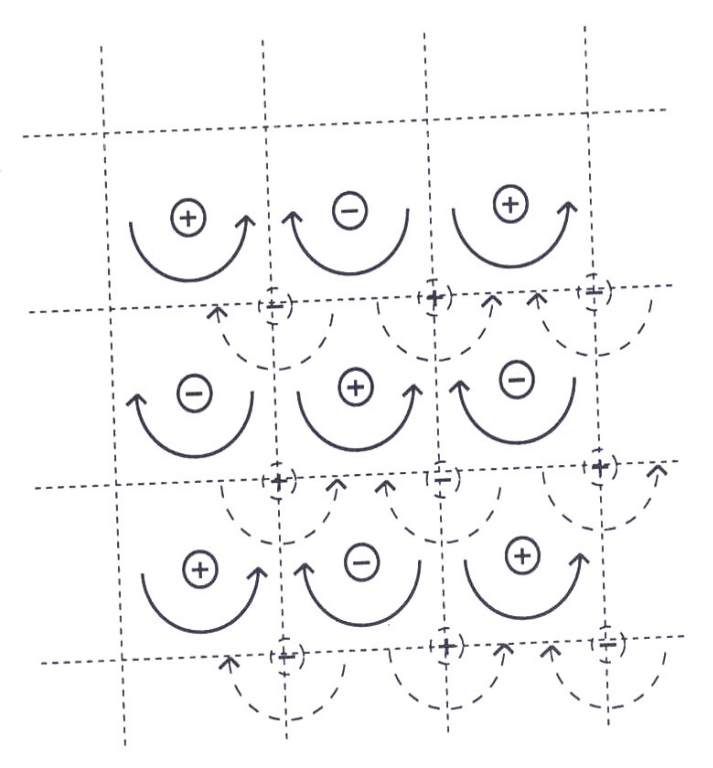

Figure 5.1: Modified velocity by Lorentz force: From Tilgner \& Busse (2001)

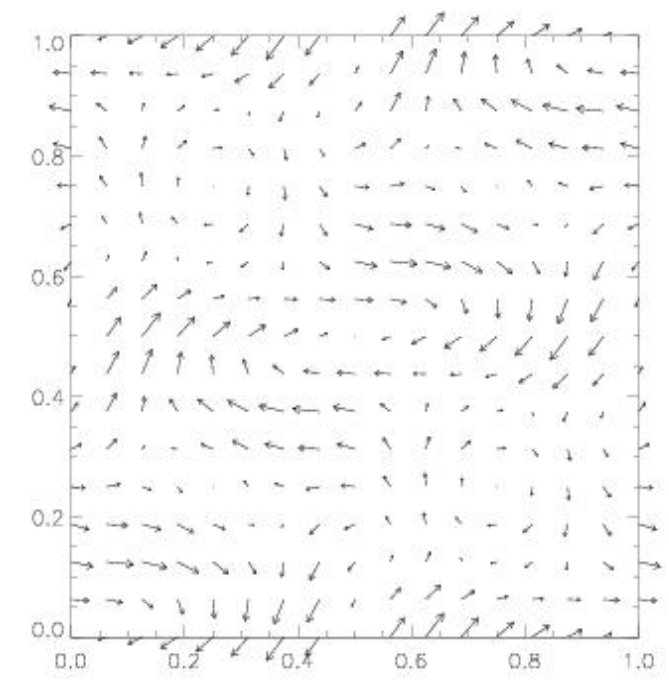

Figure 5.2: Modified velocity by Lorentz force resulting from the numerical simulation: A horizontal cross-section through $z=0.5$

this equation one has to neglect the fluid inertial term of the Navier-Stokes equation. The Alfvén wave is specially created by this inertia of the fluid. Let us assume an external field in $x$ direction so that

$$
\overline{\boldsymbol{B}}=B_{e} \hat{\boldsymbol{x}}+\tilde{\boldsymbol{b}}
$$


where $\tilde{b}$ is the wave, and it evolves like

$$
\partial_{t} \tilde{\boldsymbol{b}}+\nabla \times A\left(\begin{array}{c}
\tilde{b}_{x} \\
\tilde{b}_{y} \\
0
\end{array}\right)-\nabla \times C B_{e}^{2} \tilde{b}_{y}\left(\begin{array}{l}
0 \\
1 \\
0
\end{array}\right)=\frac{1}{R_{m}} \nabla^{2} \tilde{\boldsymbol{b}} .
$$

In the simplest case, $\tilde{b}$ depends only on $z$, and it is enough to consider only the $x$ and $y$ components. In that case the solution must be of the form

$$
\left(\begin{array}{l}
\tilde{b}_{x} \\
\tilde{b}_{y}
\end{array}\right)=\left(\begin{array}{l}
\tilde{b}_{x 0} \\
\tilde{b}_{y 0}
\end{array}\right) e^{i k z} e^{\left(-\frac{k^{2}}{R_{m}} \pm s t\right)}
$$

which leads to

$$
s=k A \sqrt{1-\left(\frac{a}{\pi}\right)^{2} \frac{R_{e} R_{m}}{2} B_{e}^{2}} .
$$

In equation (5.4) if the radicand is negative there is an oscillatory solution with a damping rate $-\frac{1}{R_{m}} k^{2}$. As is noticed for large magnetic fields the frequency is proportional to the magnetic field, as it is in the case of Alfvén waves. But the difference lies in the direction of propagation, which is perpendicular to the applied field in the case of this newly derived wave. Later in this chapter we verify the presence of this wave in the real experiment.

\subsection{Simulation of the modified velocity profile}

In our first simulation we try to simulate the non-linear dynamo using the spectral method. The result is checked with the Spectral-LBM coupled code as well. Both of them have shown the same result except that the CPU time taken by the coupled code is longer (as was mentioned in the previous chapter).

We consider a liquid conductor in a periodic box $0 \leq x \leq 1,0 \leq y \leq 1,0 \leq z \leq 1$. The equations for the non-dimensional velocity $\boldsymbol{u}(\boldsymbol{r}, t)$ and magnetic field, $\boldsymbol{B}(\boldsymbol{r}, t)$ are in exact agreement with (4.7). In order to have the same approximation as Tilgner \& Busse (2001) where fluid inertia is neglected, we keep the hydrodynamic Reynolds number $\left(R_{e}\right)$ at a very low value like 0.01 . Afterwards the magnetic Reynolds number $R_{m}$ is varied. In equation (4.7) $p$ is the non-dimensional pressure and $\boldsymbol{f}(\boldsymbol{r})$ is a force which maintains the flow against viscous dissipation and the magnetic force $(\nabla \times \boldsymbol{B}) \times \boldsymbol{B}$. Here $\boldsymbol{f}$ is chosen to be

$$
\boldsymbol{f}=\frac{8}{R_{e}} \pi^{2} \boldsymbol{u}_{0}
$$

with

$$
\boldsymbol{u}_{0}=\left(\begin{array}{c}
\sqrt{2} \sin (2 \pi x) \cos (2 \pi y) \\
-\sqrt{2} \cos (2 \pi x) \sin (2 \pi y) \\
2 \sin (2 \pi x) \sin (2 \pi y)
\end{array}\right)
$$


This drives $\boldsymbol{u}_{0}$. The flow, generated in this way, resembles the flow in the Karlsruhe Dynamo and the flow predicted by the above mentioned theoretical work.

In the simulation initially the Lorentz force was kept out i.e., the magnetic field was allowed to grow kinematically; but since the flow is not affected by any other force than the force $\boldsymbol{f}(\boldsymbol{r})$, it saturate after a certain time and so does the kinematic energy as well.

Once the Lorentz force is switched on, the dynamo becomes non-linear. The Lorentz force starts acting on the fluid flow and it changes the velocity profile and decreases the kinetic energy. Eventually, kinetic energy as well as magnetic energy saturates. The final velocity profile is shown in the figure (5.2) which has resemblances to the one in figure (5.1). Hence we can conclude that our simulation result supports the above mentioned theoretical conclusion.

\subsection{Magnetic field oscillation in simulations}

As mentioned before, the Karlsruhe Dynamo Experiment showed magnetic field oscillations about its mean value when it saturates. The mechanical structure was not implemented so far in any of the simulations. We use the lattice Boltzmann method to implement some structures in our simulation to see their effect.

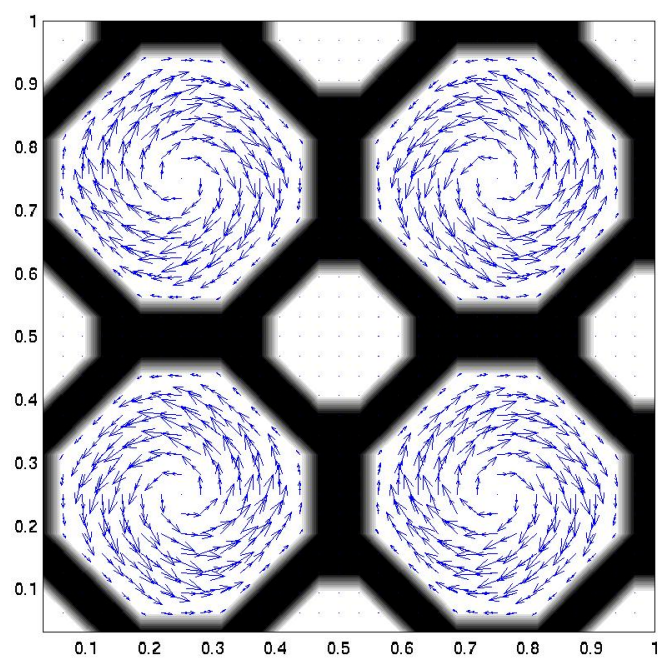

Figure 5.3: Velocity profile with walls: a horizontal cut through the middle of the box $(z=0.5)$

In the following simulation (Sarkar,Tilgner;2005b) we consider liquid sodium in a periodic box $0 \leq x \leq 1,0 \leq y \leq 1,0 \leq z \leq 1$. We introduce four cylinders with axes at $(x, y)$ co-ordinates $(0.25,0.25),(0.75,0.25),(0.75,0.25)$ and $(0.75,0.75)$. Each cylindrical wall has an inner radius of 0.2 and an outer radius of 0.25 . The boundaries are assumed 
to be no-slip and the wall material is supposed to have the same electrical conductivity as the fluid. The horizontal cross-section of the wall including the flow profile is shown in figure (5.3). Here the walls do not look like perfect cylinders, because nodal points of the simulation box are used to define the walls.

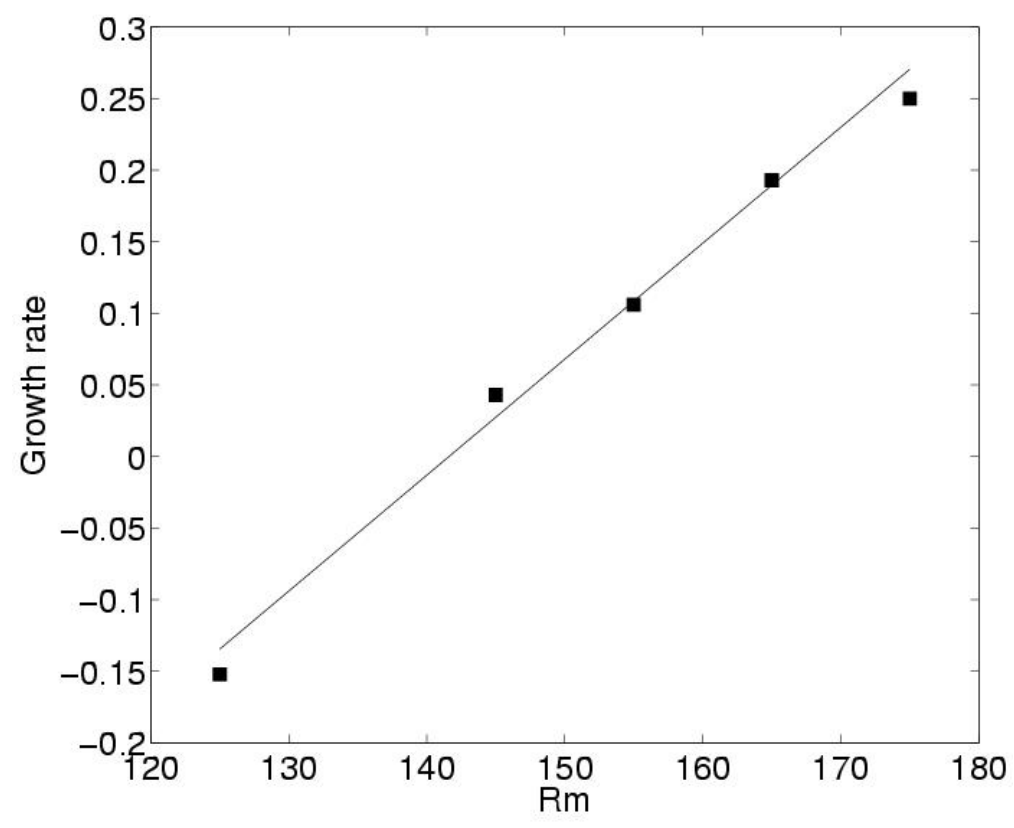

Figure 5.4: Growth rate of the magnetic energy as a function of $R_{m}$

The equations are the same as equation (4.7). We fix the hydrodynamic Reynolds number $R_{e}$ at 100 and the magnetic Reynolds number $R_{m}$ is varied. $\boldsymbol{f}(\boldsymbol{r})$ is a force which maintains the flow against viscous dissipation and the magnetic force $(\nabla \times \boldsymbol{B}) \times \boldsymbol{B}$. $\boldsymbol{f}$ is chosen in such a way that it maintains the flow $\boldsymbol{u}_{0}$ (in the absence of walls), which gives us in correspondence to equations (5.7) and (5.6)

$$
\boldsymbol{f}=\frac{8}{R_{e}} \pi^{2} \boldsymbol{u}_{0}
$$

with

$$
\boldsymbol{u}_{0}=\left(\begin{array}{c}
4 \sin (2 \pi x) \cos (2 \pi y) \\
-4 \cos (2 \pi x) \sin (2 \pi y) \\
2 \sin (2 \pi x) \sin (2 \pi y)
\end{array}\right)
$$

The force $f$ is set to zero at grid points inside the cylindrical walls and the interstitials between the cylinders.

The flow thus generated, resembles the Karlsruhe dynamo in that it consists of an array of eddies with circular cross section. The fluid in between these eddies is not forced in the simulation or pumped in the experiment, but it can be set into motion by the Lorentz force once a magnetic field appears. The walls keep the eddies in place so that they cannot 


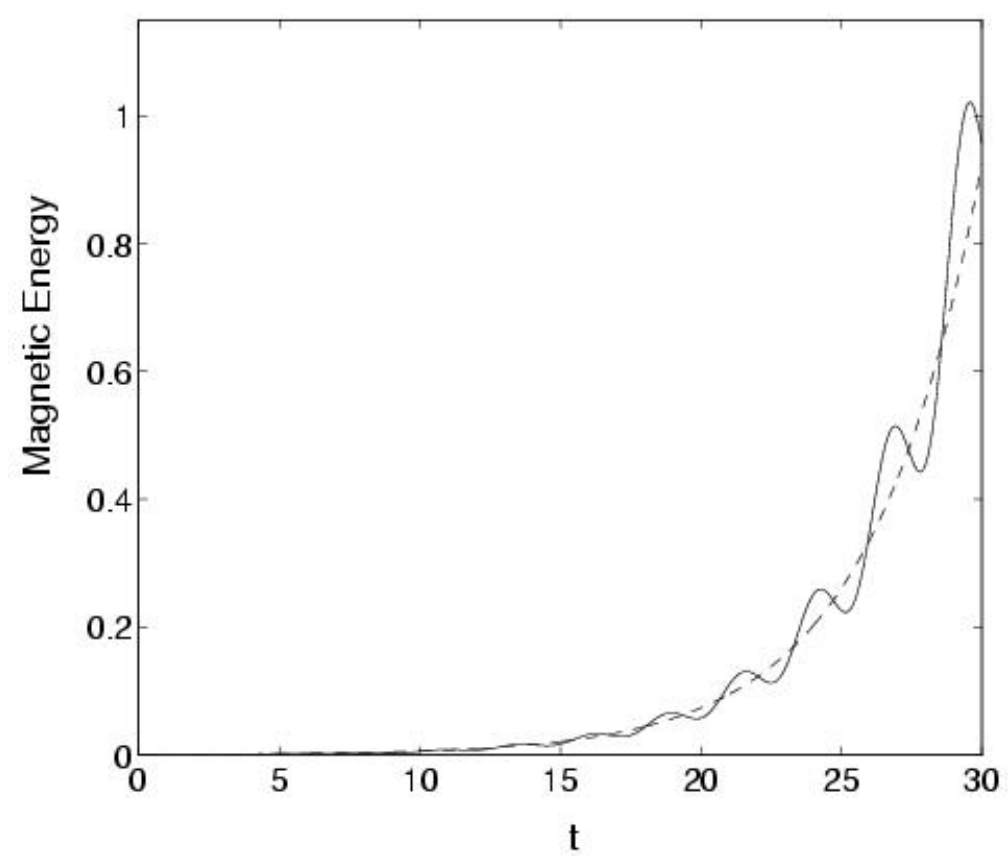

Figure 5.5: Kinematic growth of the magnetic field energy for $R_{m}=175$. The dashed line is the best exponential fit

bend or distort considerably as they do in simulations without any walls. Obvious discrepancies remain between this model and the real experiment. There is still less mechanical structure included in the simulation than is present in the experiment, and the experiment contains only 52 eddies instead of an infinity of them. The aspect ratio chosen in the simulation is not realistic in the sense that the ratio of the cell height to eddy diameter is approximately 5 in the experiment.

The simulations have been started from fluid at rest and a weak uniform magnetic field in $x$ direction. The spatial resolution was $\frac{1}{32}$. Let us then first consider the kinematic problem in which the $(\nabla \times \boldsymbol{B}) \times \boldsymbol{B}$ is dropped from equation (4.7c). There is then no back-reaction of the magnetic field on the flow and the magnetic field either decays to zero or grows indefinitely. In these simulations, the magnetic energy was monitored as a function of time. The time dependence of this energy was fitted to an exponential at times greater than the time the velocity field needs to reach equilibrium. It is concluded from the growth rate as a function of $R_{m}$ that the critical magnetic Reynolds number, $R_{m_{c}}$ is equal to 142 (fig. 5.4). The time dependence of the magnetic energy is not strictly exponential but there are oscillations with a well defined period around the average exponential growth or decay (fig. 5.5). The period of these oscillations is shown as a function of $R_{m}$ in figure (5.6).

Large portions of the fluid are stagnant so that $R_{m_{c}}$ is much higher than in simulations in which the force (5.7) is used in the entire computational volume and in the absence of walls. In that case (the previous simulation), the critical magnetic Reynolds number is 16 and there are no oscillations. However, the oscillations are not simply due to a high 


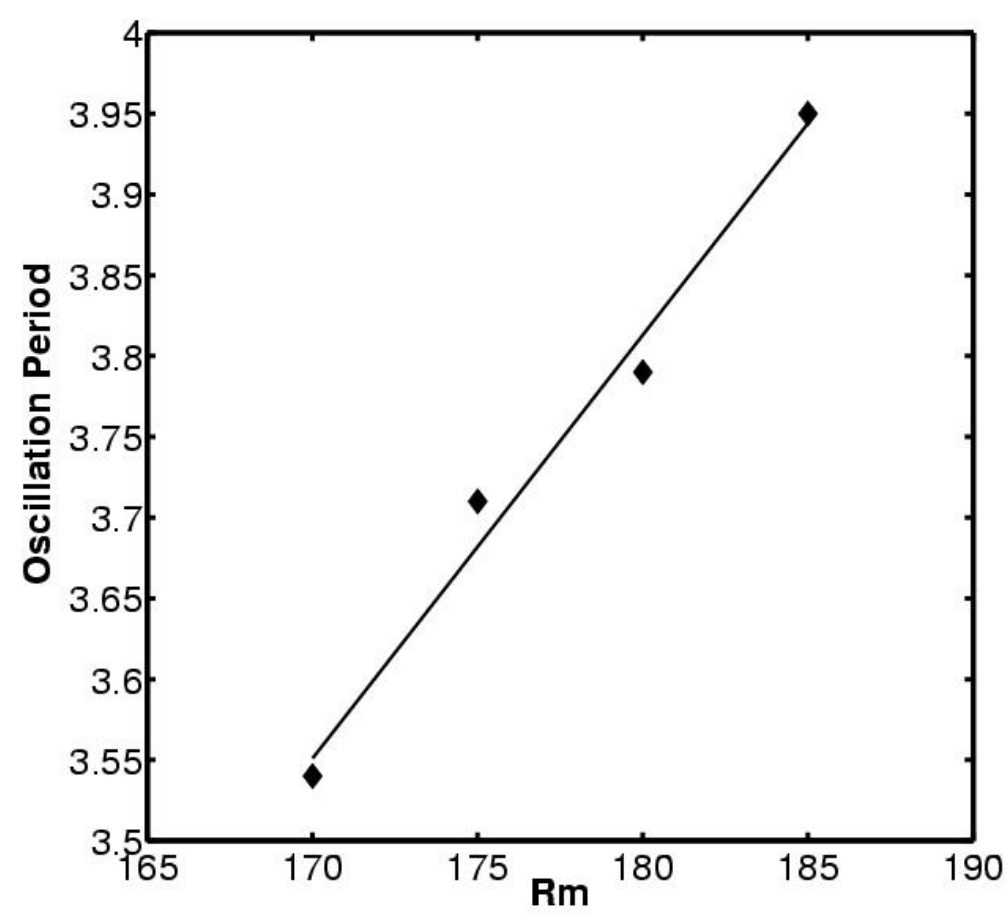

Figure 5.6: Dimensionless period of the magnetic energy oscillations vs. magnetic Reynolds numbers
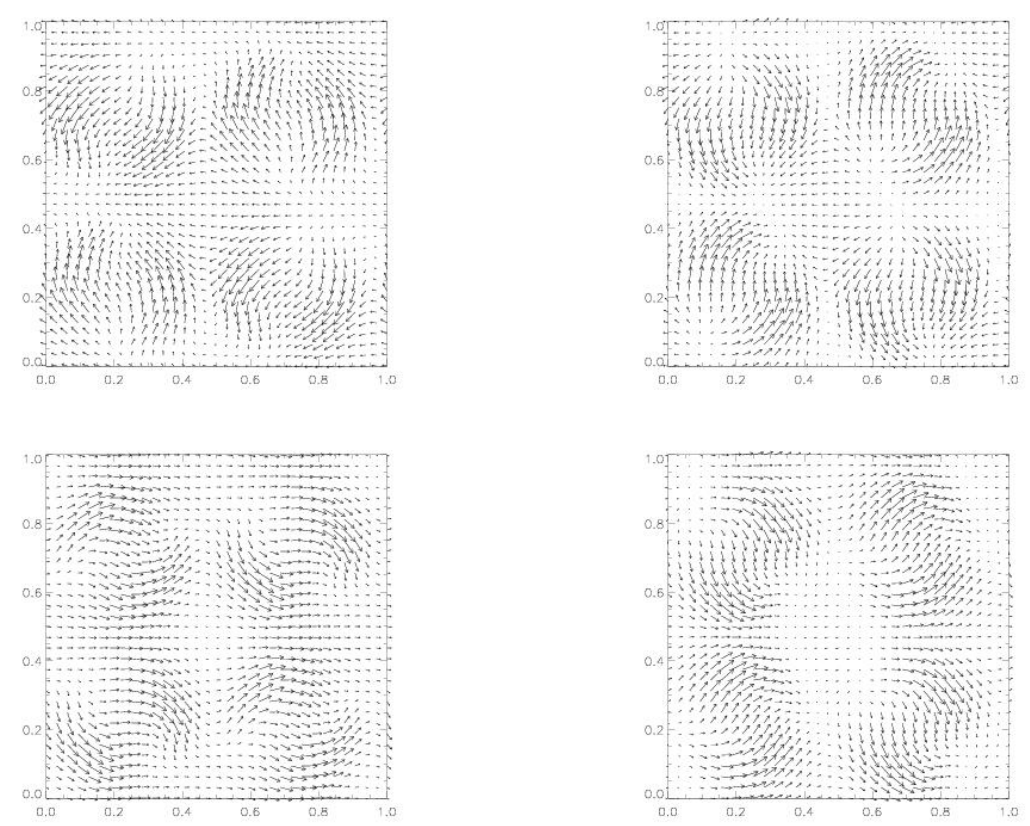

Figure 5.7: The four plots cover one complete period of the oscillation, starting in the upper left panel and continuing clockwise. The arrows indicate the $x-$ and $y$-components of the magnetic field at $z=0.5$ 

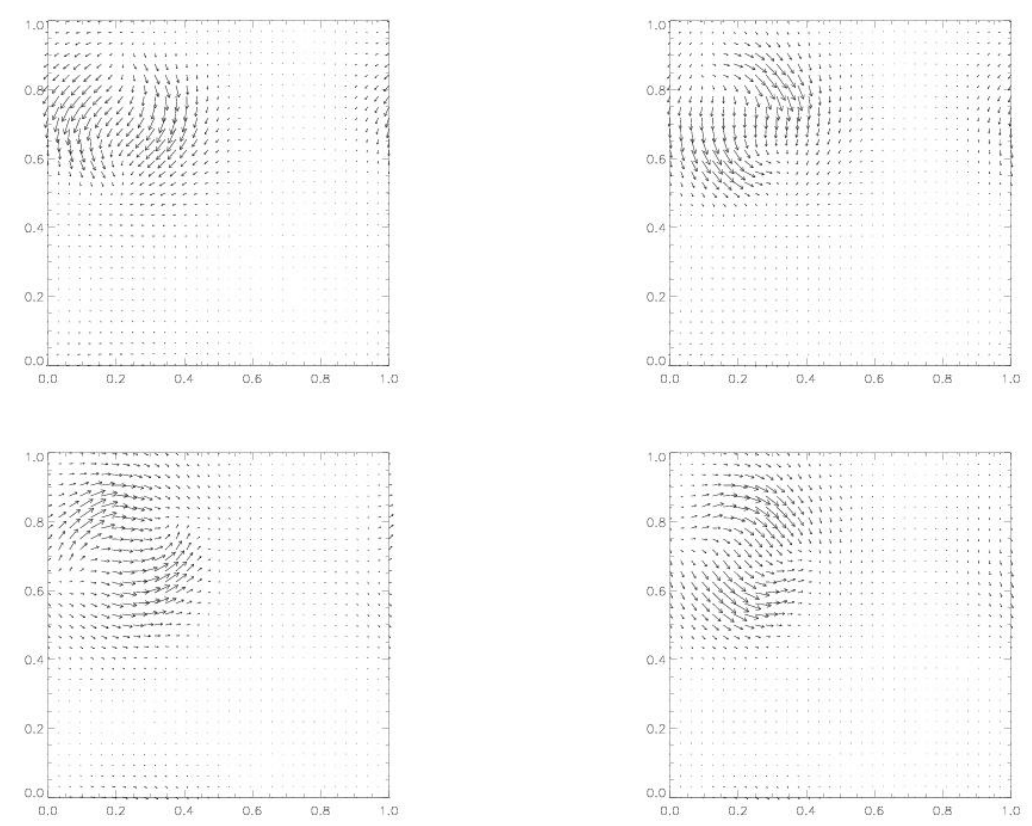

Figure 5.8: The magnetic field evolution keeping one single vortex alive. The first picture is the upper left panel and subsequent plots are ordered in clockwise sense. The arrows indicate the $x-$ and $y$-components of the magnetic field at $z=0.5$

$R_{m}$. The simulations without walls did not show oscillations at $R_{m}=180$ either, so that the velocity profile must be responsible for the appearance of the oscillations. In another simulation, the full equations (4.7c) and (4.7a) have been integrated for $R_{e}=100$ and $R_{m}=175$. Figure (5.7) shows snapshots of the magnetic field separated by equal time intervals covering half a period of the oscillation of the magnetic field energy. It is seen that the field pattern inside each of the cylinders rotates about the axis of the cylinder.

Kinematic simulations of comparable velocity fields have so far only yielded purely real growth rates, i.e. no oscillations (G. O. Roberts 1972; Tilgner \& Busse 1995). Traveling waves occurred only in examples where the $z$-component of $\boldsymbol{u}_{0}$ is shifted with respect to the in-plane components or otherwise modified so that the flow does not have an $\alpha$-effect. A homogeneous $\alpha^{2}$-dynamo, on the other hand, must produce non-oscillatory magnetic fields. It is thus surprising that oscillations are introduced by the inclusion of walls in the simulation which at first sight merely changes the velocity profile within every helical eddy but does not destroy periodicity, helicity and other general features.

Figure (5.7) has shown that the oscillations are due to a rotation of the magnetic field. This rotation is not simple advection of the magnetic field lines because the apparent sense of rotation in figure (5.7) is exactly opposed to the rotation of the fluid in each eddy. It is also noteworthy that the local field in the eddies is larger than the mean field one obtains by averaging the magnetic field over planes $z=$ const.. It seems therefore that this dynamo does not operate as an $\alpha^{2}$-dynamo, but instead consists of four nearly indepen- 

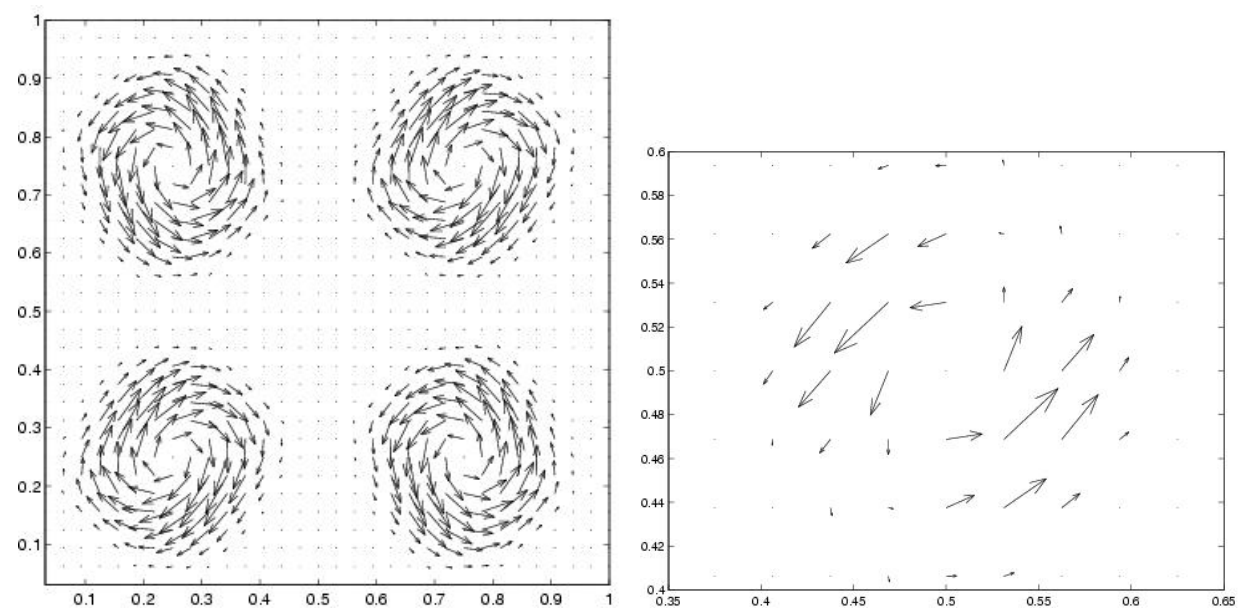

Figure 5.9: The left panel shows the subtracted (velocity modified by the Lorentz force minus velocity without magnetic field) velocity profile and the right panel shows the vortex at the junction of the four cylinders

dent Ponomarenko dynamos (Ponomarenko 1973). Indeed, an isolated helical vortex in an infinite volume of otherwise quiescent fluid is also a dynamo. A possible numerical approach to such a dynamo would be to put one helical vortex in a periodic box as long as the periodicity length is large compared with the diameter of the vortex. It must thus be possible to go from an $\alpha^{2}$-dynamo to isolated Ponomarenko dynamos by continuously decreasing the eddy size and concomitantly increasing the interstitial volume which is filled with fluid at rest. Comparing the velocity field $\boldsymbol{u}_{0}$ originally considered by G. O. Roberts and the velocity field obtained in the present simulations when the cylindrical walls are included, one sees that the latter case is closer to four independent vortices than the former. A cursory look at the velocity field suggests that it still should behave like an $\alpha^{2}$-dynamo because the volumetric fraction of stagnant fluid is fairly small, but the simulations show that it is already large enough to magnetically decouple the four vortices.

Probably there is still very little connection between four individual vortices. But after the dynamo saturates we see a feeble vortex (fig. 5.9) at the junction of these vortices where the fluid was stagnant initially. This is be expected if the dynamo were a real G. O. Roberts dynamo. But in the G. O. Roberts dynamo this vortex is much stronger.

The question that arises in this case is- what is the effect of the magnetic field saturation on the overall velocity profile? In figure (5.9) we present the subtracted velocity profile (velocity without magnetic field minus the velocity resulted from the back-reaction) which shows that every vortex gets an oval shape.

So far it is believed here that in our domain there are four individual Ponomarenko dynamos which give rise to the oscillation. To confirm the idea we keep only one vortex alive and make others to zero in the same simulation setup. This kind of simulation also shows the rotation of the magnetic field (fig. 5.8) as is expected from the Ponomarenko dynamo. At $R_{m}=175$ the rotation period of the magnetic field structure is 7.4 , which is 
also observed when all four vortices are active.

\subsection{Rotating wall simulation}

In the previous simulation, because of wall friction the fluid was slow enough near to the wall, which made the onset of the dynamo at much higher $R_{m}$. In the experiment, of course, the wall is present, but the wall thickness is much smaller than the one in our previous simulation. Thinner walls could be implemented, but that makes the computational cost too high. To bring down the onset of the dynamo, we implement rotating walls in this simulation. This keeps some part of the fluid static (outside vortices) initially. Our main aim is to see the back-reaction effect on this static part of the fluid. We do this simulation in a $32^{3}$ box, keeping four cylinders inside so that each cylinder can generate one single vortex. There is no external horizontal force to drive the fluid. The inner walls of these cylinders are given rotation, leaving the outer ones static, so that the fluid attached to the inner part of each cylinder gets a rotational motion while the outer part does not. There is an external uniform forcing in vertical direction over every cylinder to get the helical motion of the fluid inside. The rotation of the cylinders and the vertical forcings are managed in such a fashion that the overall velocity profile gets close to the G. O. Roberts one.

The simulations have been started from fluid at rest and a weak uniform magnetic field (along $x$ direction). But soon a large part of the fluid can be in motion due to the rotating walls, and simultaneously the onset of the dynamo can be brought down from $R_{m}=142$ in the previous simulation to $R_{m}=66.2$ kinematically. In figure (5.11) we plot the growth rate vs. $R_{m}$ of this simulation.

\subsubsection{Subcritical onset}

Usually the critical magnetic Reynolds number $\left(R_{m_{c}}\right)$ for the onset of the dynamo is independent of whether the dynamo simulations are without or with Lorentz forces. But in this simulation the $R_{m_{c}}$ is different in the non-linear case from that for the kinematic dynamo simulation.

We start the simulation in the kinematic supercritical domain i.e. $R_{m}>66.2$, keeping the Lorentz force on. This allows the Lorentz force to act on the velocity field. This changes the overall velocity profile (fig. 5.10) in such a way that the magnetic field saturates eventually. During saturation the induction equation looks like

$$
0=\nabla \times(\boldsymbol{v} \times \boldsymbol{B})+\frac{1}{R_{m}} \nabla^{2} \boldsymbol{B} .
$$

Now, if the output of the supercritical $R_{m}$ simulation is used as an initial condition for the simulation with subcritical $R_{m}$, the magnetic field decays initially and then saturates again above a certain $R_{m}$. We vary $R_{m}$ in the subcritical domain until the saturated magnetic field goes down to zero. This happens at $R_{m}=47$, and we name it subcritical onset. 


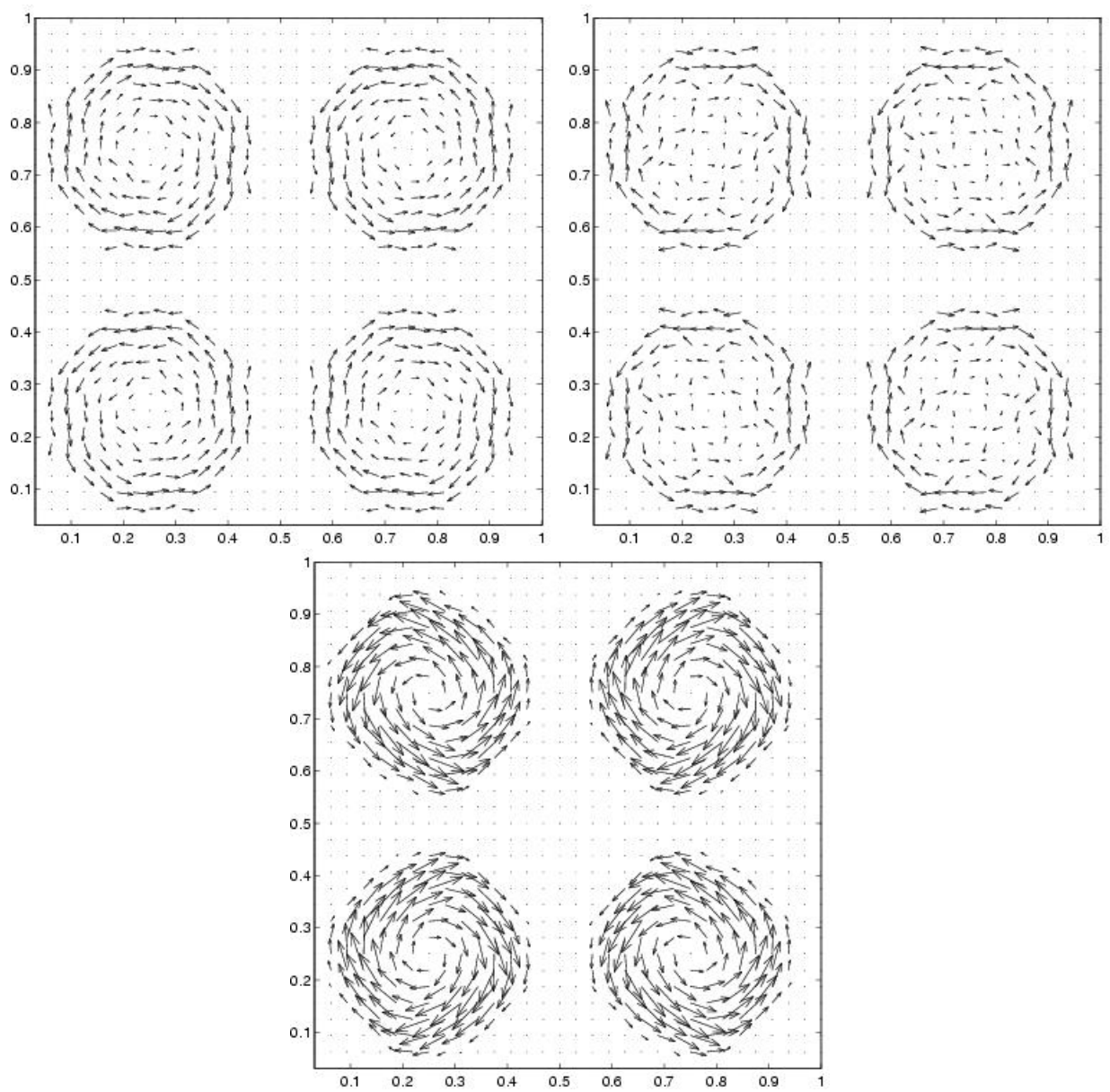

Figure 5.10: Rotating wall simulation: Velocity field before (left) and after (right) affected by the Lorentz force. In the bottom panel the subtracted (left minus right) velocity profile is shown

Between kinematic onset and subcritical onset we run the simulation kinematically as well with the saturated velocity profile modified by the Lorentz force. This shows zero growth rate of the magnetic field. This is also expected from the equation (5.9). This equation is satisfied for $\boldsymbol{v}=\boldsymbol{v}_{\text {sat }}$, so when one takes $\boldsymbol{v}_{\text {sat }}$ as an input of the equation the growth rate remains zero. In figure (5.12) we plot the saturated magnetic energy $\left(E_{B_{s a t}}\right)$ vs. $R_{m}$ which shows that the magnetic energy decreases with decreasing $R_{m}$ and below $R_{m}=48$ it is zero. Here magnetic energies are calculated using trapezoidal rule for integration. The formula for the magnetic energy $\left(E_{B}\right)$ is the same as equation (4.10).

\subsection{On the MHD instability of the G. O. Roberts flow}

The motivation of this simulation is to find the origin of the magnetic field oscillation as observed in the Karlsruhe Dynamo Experiment. We take the simple G. O. Roberts flow as is mentioned in equation (5.6). and try to see the back-reaction effect on the flow. For 


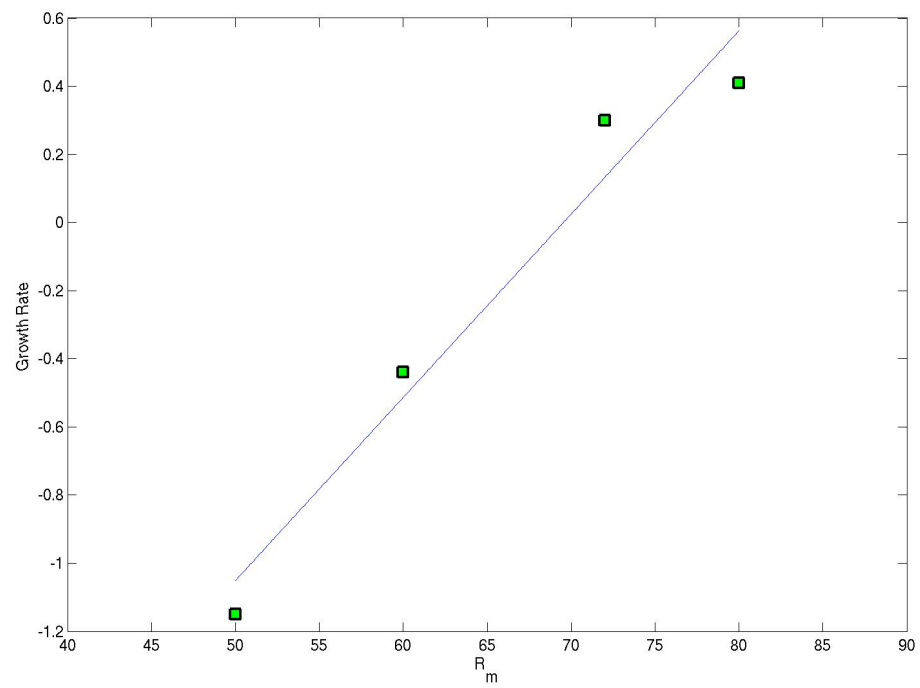

Figure 5.11: Growth rate of the magnetic energy as a function of $R_{m}$ for the rotating wall simulations

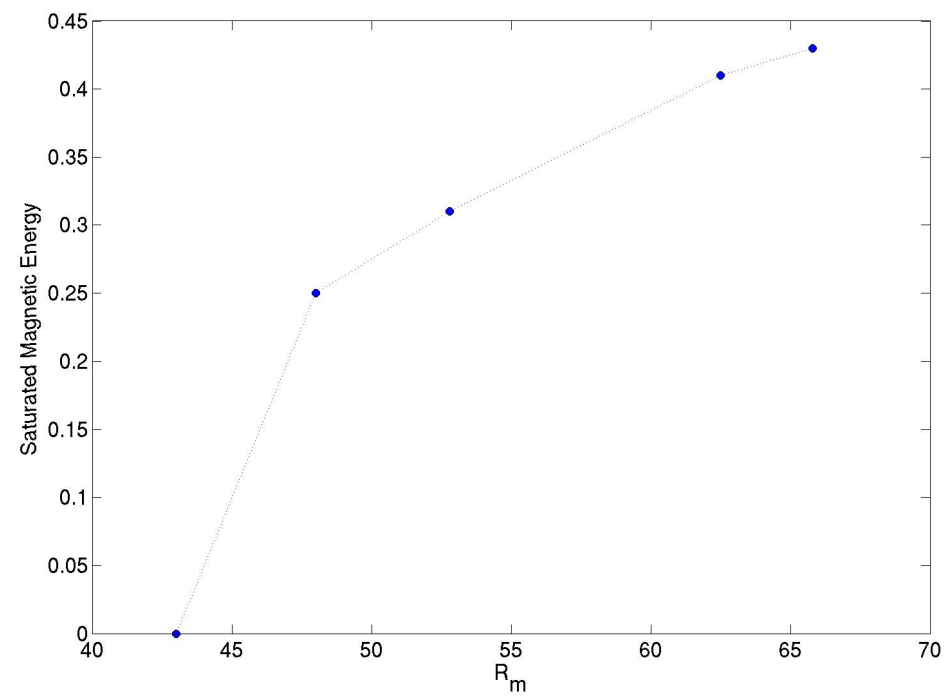

Figure 5.12: Subcritical onset: Saturated magnetic energy is plotted against $R_{m}$ 


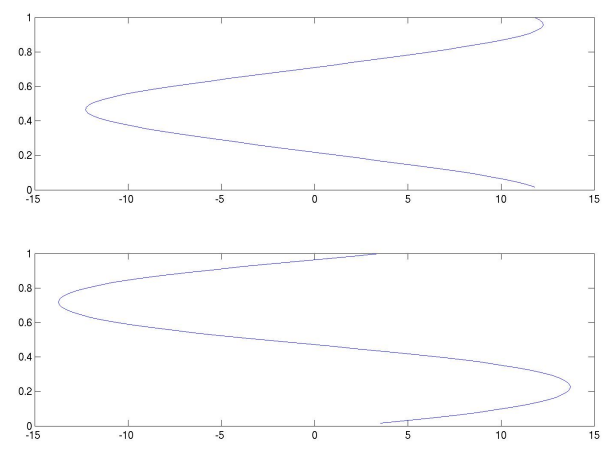

Figure 5.13: The mean field structure of the simulated magnetic field: The upper panel shows the $\mathrm{x}$ component of the mean magnetic field and the bottom panel shows the $\mathrm{y}$ component of the mean magnetic field. Both are plotted against $z$ axis (The mean is taken over $x$ and $y$ co-ordinates)

this simulation we use the spectral method to simulate a whole dynamo as there is no wall required. The prime question which appears first is whether the quenching effect mentioned above provokes the oscillation.

This simulation is exactly the same as for our very first dynamo simulation but in a box of $64^{3}$. Initially, for low $R_{m}$ the flow is steady and does not show any oscillation even after saturation. There is a kind of oscillation which starts at higher $R_{m}$; the onset of this is at $R_{m}=80$. Initially, the oscillation is periodic with increasing period (i.e, decreasing frequency), when $R_{m}$ increases. But later at still higher $R_{m}$ the oscillation becomes chaotic. This fact is clearly against the experimental observation. Interpreting the figure (2.5) one concludes the experimental oscillation frequency increases with increasing $R_{m}$, i.e, from $1.2 \mathrm{~Hz}$ for $R_{m}=8.96$ to $2.7 \mathrm{~Hz}$ for $R_{m}=11.4$. The mean field of the simulation is calculated by taking the time averages over a period of oscillation and then this mean magnetic field is plotted by taking spatial average over $x$ and $y$ components. In figure (5.13) the $z$ dependence of the mean field $(\bar{B})$ is shown. The fluctuating part $\left(\boldsymbol{B}^{\prime}\right)$ shows movement but is not regular as evidenced in figure (5.14).

$\begin{array}{lll}R_{m} & \begin{array}{l}\text { Dimensionless } \\ \text { oscillation period }\end{array} & \begin{array}{l}\text { Dimensionless } \\ \text { amplitude }\end{array} \\ 65 & 7.892 & 2.4 \\ 75 & 9.08 & 12.065 \\ 80 & 10.05 & 18.15 \\ 100 & 30.49 & 36.7\end{array}$

Table (5.1): Oscillation characteristics

It seems that the oscillation of the magnetic field is initial-condition-dependent. This could not be observed at comparatively low $R_{m}$ (eg. $R_{m}=60$ ), but at higher $R_{m}$ (say, 150 ) it is clearly visible when the magnetic energy is plotted. Once the output of the 

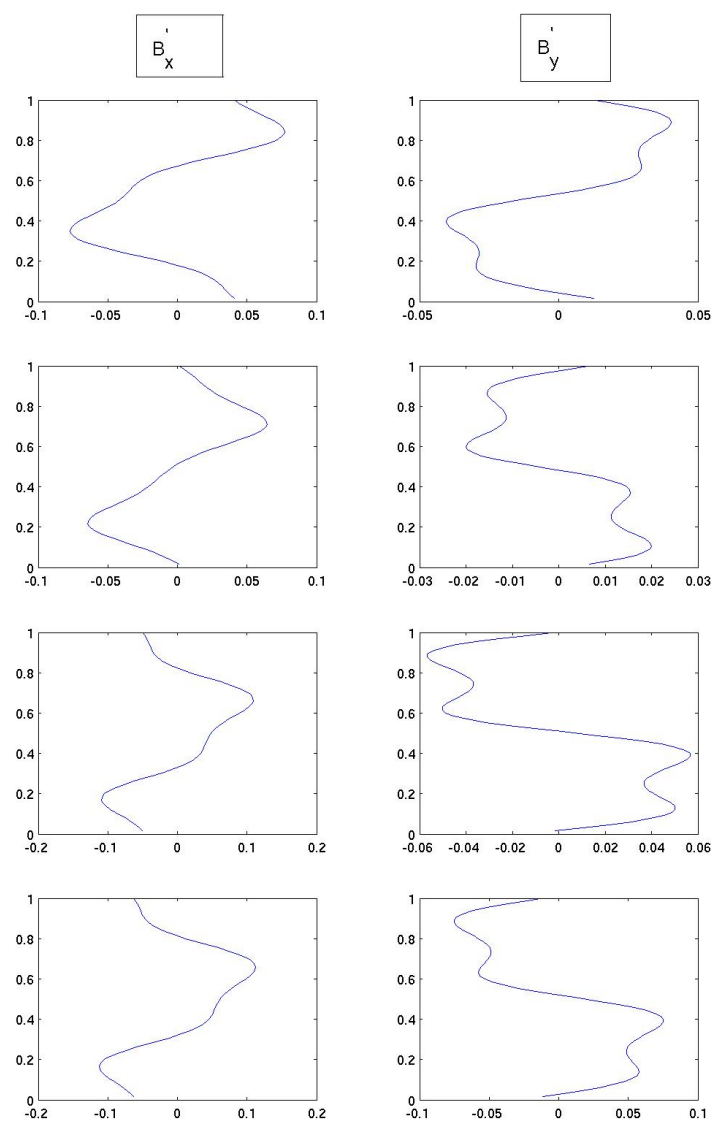

Figure 5.14: Evolution of the fluctuating magnetic field over a period of oscillation: $B_{x}^{\prime}$ and $B_{y}^{\prime}$ are the $x$ and $y$ components of the fluctuating magnetic field $\boldsymbol{B}^{\prime}$; both are plotted against the $z$ axis (average is taken over $x$ and $y$ co-ordinates)

higher $R_{m}$ simulation is brought to the lower $R_{m}$ simulation as initial condition, the instability is observed at low $R_{m}$ as well. From the table (5.1) it is seen that amplitude of the magnetic field oscillation at $R_{m}=65$ is 2.4 , and it goes to zero at $R_{m} \sim 60$ if extrapolated. It is checked as well that there is no hysteresis once the instability sets in, i.e. if the output of one simulation is taken into the subsequent simulation of different $R_{m}$, the oscillation remains unchanged.

\subsubsection{G. O. Roberts dynamo with changing superimposed perturba- tions}

Since the above mentioned oscillation has no agreement with the experimental oscillation, one has to generate a new way to discover the latter. It is believed that the experimental oscillation is due to the fact that there is some kind of interaction of the magnetic field and local eddies. These local eddies might be the result of the high $R_{e}$ flow through 

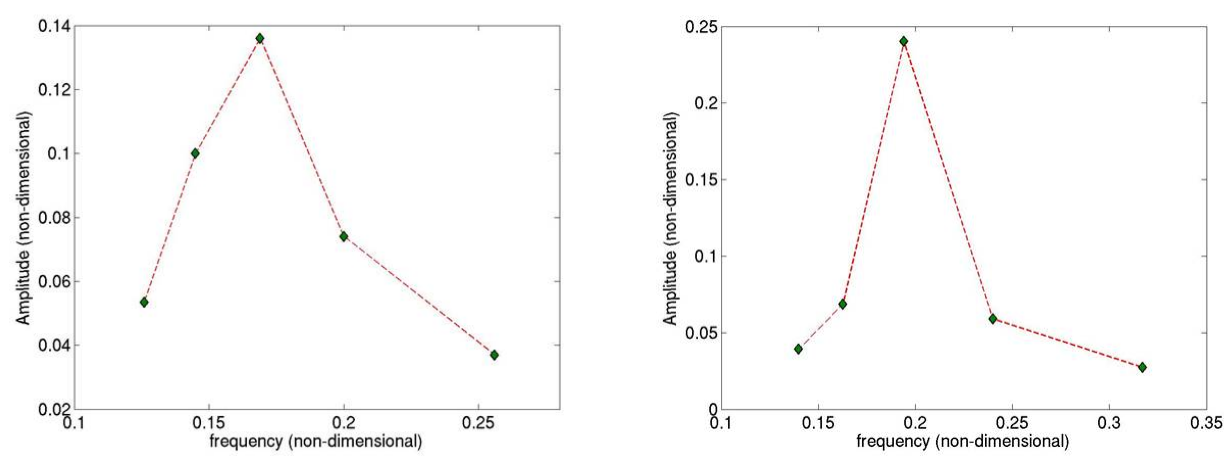

Figure 5.15: Amplitude vs. Frequency : for two different $R_{m}, 60$ (left) and 70 (right)

complicated mechanical structure. Thus one is provoked to introduce some local eddies in the simulation box. Keeping this in mind we start our simulation with the G. O. Roberts velocity profile but along with that a time-harmonic velocity perturbation function $\boldsymbol{g}(\boldsymbol{r}, t)$,

$$
\boldsymbol{g}(\boldsymbol{r}, t)=\boldsymbol{f}(\boldsymbol{r}) \sin (2 \pi f t)
$$

with

$$
\boldsymbol{f}(\boldsymbol{r})=\left(\begin{array}{c}
A \sin (2 \pi x / b) \cos (2 \pi y / b) \\
A \cos (2 \pi x / b) \sin (2 \pi y / b) \\
A \sin (2 \pi x / c) \sin (2 \pi z / c) .
\end{array}\right)
$$

This represents local eddies of scale length $b$ and $c . A=0.3$ in this case and $b, c$ were varied during simulation, which will be discussed soon.

Since the grid resolution is not affecting the result much, we choose a spatial resolution $d x=\frac{1}{16}$. But our aspect ratio in this simulation is $1: 1: 2$, which brings down the onset of the dynamo at lower $R_{m}$. So this simulation is done in a box $16: 16: 32$.

The time component of the perturbation is implemented keeping in mind that if there is some kind of oscillation which is invisible and if the frequency of that oscillation is matched with the frequency of the time perturbation $f$, it will probably show up as resonance. To find out the oscillation frequency we try our simulation for several $f$. The spatial and the time perturbation are repeated over every time step. Since the dynamo becomes unstable by itself at high $R_{m}$ we try to concentrate in the lower $R_{m}$ region with $R_{m}$ below 80 , where the dynamo is stable otherwise. The time component of the perturbation disturbs the flow from the beginning and hence the kinetic energy and the magnetic energy both show the oscillation with the same frequency $f$. The $R_{e}$ of the flow is kept sufficiently low (within 10), and we shall discuss the $R_{e}$ dependence later.

In figure (5.15) we plot amplitude vs. frequency, $f$ of the oscillation for two different $R_{m}$ (70\&60). This clearly shows that there is a peak at $f=0.19$ for $R_{m}=70$ and $f=0.17$ at $R_{m}=60$. But for smaller $R_{m}$ the peak almost vanishes. This is probably because the oscillation is very weak at low $R_{m}$. We try to plot in figure (5.16) frequency $f$ in Hertz vs. $R_{m}$ and once it is extrapolated it can be noticed that the onset of the oscillation 


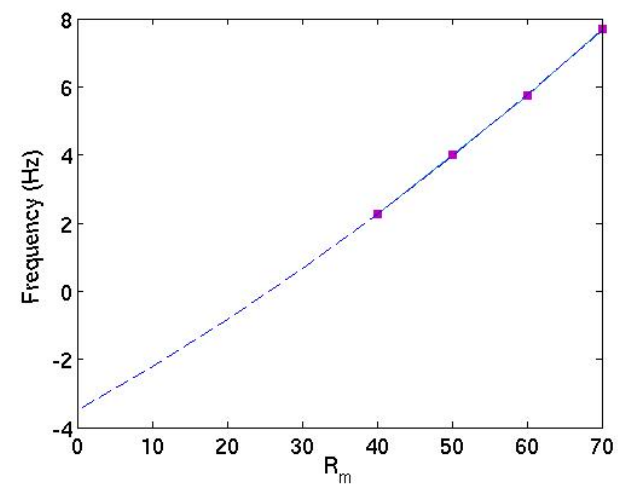

Figure 5.16: Oscillation Onset: frequency vs. $R_{m}$ of the oscillation, pink squares denote the simulated oscillation frequency, the dashed line is used to extrapolate the simulated oscillation frequency

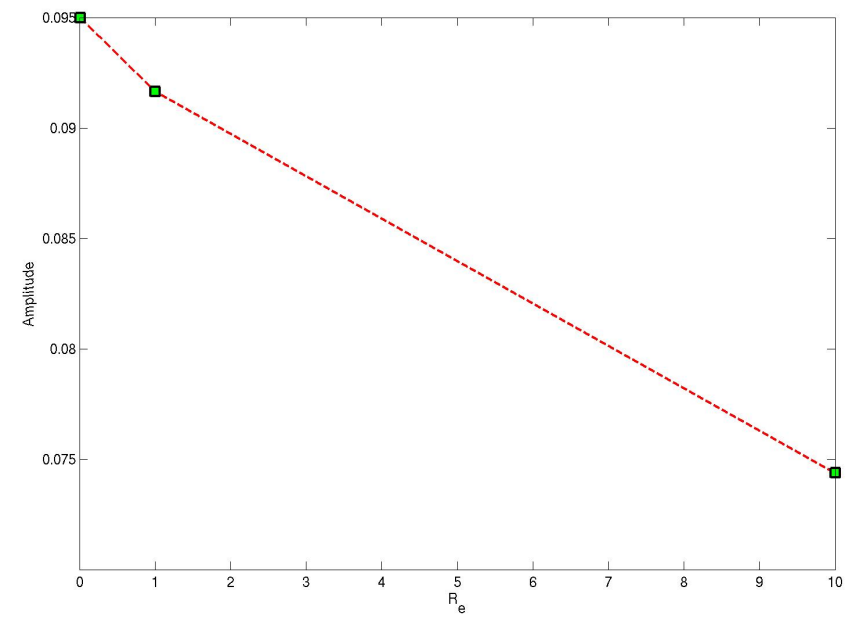

Figure 5.17: Amplitude of oscillation at different $R_{e}$ for a fixed $R_{m}$

is at around $R_{m}=25$. This is far above the dynamo onset, which is at $R_{m}=8$.

In one sense this result agrees with the experimental oscillation for which the spectral peak $f_{p}$ decreases along with the decreasing $R_{m}$ or flow rate. In figure (5.16) one sees that at $R_{m}=40$ the frequency of this oscillation is $2.27 \mathrm{~Hz}$., and thus close to the spectral peak position in the real experiment. It should be mentioned that the value of $b$ and $c$ in equation (5.11) matters much to influence the amplitude of the resulting oscillation. The nature of $\boldsymbol{f}(\boldsymbol{r})$ is also an influencing agent to play a role here. Keeping in mind that larger eddies have lower dissipation rates we take $b=0.5$ and $c=1.0$. The nature of $\boldsymbol{f}(\boldsymbol{r})$ has also been tried with various combinations of $x, y, z$ dependences. But (5.11) has proved to be the best of all to produce resonances of higher amplitudes.

To see the effect of the $R_{e}$ on the oscillation, we conduct our simulations for $R_{e}=$ 


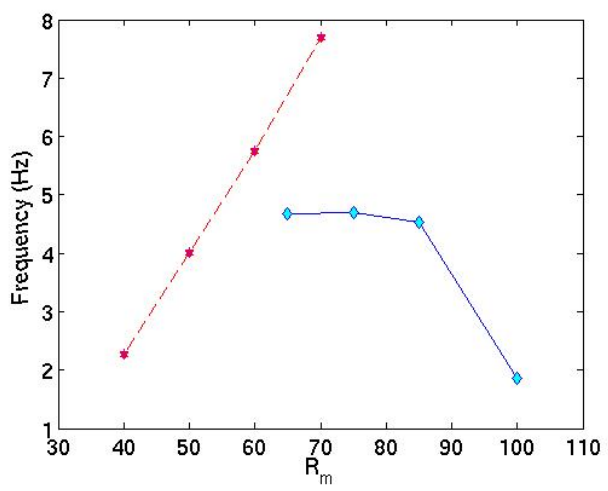

Figure 5.18: Plot showing the characteristics of two kind of oscillations: The red curve shows the region where the dynamo is stable, whereas the blue part shows the nature of the magnetic field oscillation in the unstable region of the dynamo

0.01, 1 and 10, keeping the $R_{m}$ fixed, which shows that amplitudes of the resulting oscillation are varying by 24 percent. In figure (5.17) we plot the amplitude of the oscillation at $R_{m}=50$ for these three combinations of $R_{e}$, when the imposing oscillation period is 7.0. From equation (5.4) one expects the wave generated through alpha quenching would have a frequency proportional to the saturated dynamo magnetic field $\left(B_{e}\right)$ and this wave would be independent of the hydromagnetic Reynolds number $\left(R_{e}\right)$. From table (5.2) one sees that the frequency of the simulated oscillation is not proportional to the saturated magnetic field $\left(B_{e}\right)$. Hence the origin of this wave is not the same as that derived from the analytical calculation.

\begin{tabular}{|r|r|r|r|r|}
\hline$R_{m}$ & $R_{e}$ & $B_{e}$ & $f=\frac{1}{T}$ & $\frac{B_{e}}{f}$ \\
\hline \hline 40 & 1 & 6.35 & 0.1 & 63.5 \\
\hline 50 & 1 & 6.23 & 0.14 & 44.5 \\
\hline 60 & 1 & 6.15 & 0.17 & 36.17 \\
\hline
\end{tabular}

Table (5.2): Table to show that the ratio $\frac{B_{e}}{f}$ varies significantly for different $R_{m}$

\subsubsection{Discussion}

Finally one has to be sure about the source of the magnetic field oscillation in the real experiment. We explore a possibility for the origin of the magnetic field oscillation using our analytical calculation. The frequency of the oscillation can be derived from equation (5.4). It is also mentioned earlier that the magnetic field oscillates only if the radicand of equation (5.4) is negative. To explore more about this oscillation we take the dimensional form of equation (5.4) which is

$$
\hat{s}=-\lambda \hat{k}^{2}+\hat{k} \hat{A} \sqrt{1-\left(\frac{\hat{a}}{\pi}\right)^{2} \frac{1}{\lambda \nu} \frac{\hat{\boldsymbol{B}}^{2}}{\rho \mu_{0}}} .
$$


Here all $\hat{\langle.\rangle}$ quantities are dimensional variables, with $\hat{\boldsymbol{B}}$ being the dimensional magnetic field and considering $\nu$ being the turbulent viscosity. Choosing all parameters close to the experimental values, as for example, when we take $a=0.21 \mathrm{~m} ., \lambda=\nu=0.1 \mathrm{~m}^{2} \mathrm{~s}^{-1}$, $\rho=927 \mathrm{~kg} \cdot \mathrm{m}^{-3}, \boldsymbol{B}=3 \times 10^{-3} \mathrm{~T}, \mu_{0}=1.2566 \times 10^{-6} \mathrm{Hm}^{-1}$ we find the radicand is coming out to be 0.99 , which is a positive quantity and hence we can conclude that the presence of such oscillation is impossible in the real experiment.

At the end of section (5.6.1) we have concluded that the oscillation in our last simulation is not related to the wave resulting from the analytical calculation, but has a similar nature in the sense that its frequency decreases with decreasing $R_{m}$ as it does in the experiment. On the other hand when at higher $R_{m}$ the magnetic field oscillates around its mean value, then the oscillation frequency decreases with increasing $R_{m}$. One could have doubt that these two oscillations are part of the same mechanism. Therefore we plot in figure (5.18) the frequency in Hertz of both oscillations vs. $R_{m}$ and we do not find any merging point between them. Following this we classify two distinct regions, one where frequency increases with increasing $R_{m}$, the other where it decreases with increasing $R_{m}$. In this region for very high $R_{m}$ the fluctuation of the magnetic field becomes chaotic and does not hold any regular pattern, so we can consider it to be magnetohydrodynamically unstable. This unstable region can be brought down to the stable region of $R_{m}$ if the initial velocity field is taken from the higher $R_{m}$ where the dynamo is already unstable, that is why we notice in figure (5.18) at $R_{m}=65$ even the instability is still continuing. Hence one can conclude that most probably the spectral peak of the experiment can show its observed character only up to certain $R_{m}$, above that the magnetic field becomes unstable by itself. There is a discrepancy between the experimental magnetic field oscillation and the oscillation in the stable dynamo region: the oscillation in the experiment starts close to the dynamo onset, whereas in the simulation the oscillation is non-existing close to the dynamo onset. So one is still not sure whether both oscillations have the same or different origins.

The simulated oscillation is initiated by a perturbation in space and time. These perturbations are continued over every time step. The time perturbation is used to find the frequency of the oscillation. It seems the spatial perturbation initiates the oscillation, but in our simulation we have introduced only some combinations of the spatial perturbations. If eddies of all scale lengths would be included altogether in the simulation domain, one might expect a more prominent oscillation, because out of these scale lengths some could be more efficient to initiate the oscillation than others. 



\section{Summary and Outlook}

In this thesis we concentrate to explain the magnetic field oscillation mechanism in the Karlsruhe Dynamo which is basically a G. O. Roberts dynamo. In our various simulations we try to reproduce G. O. Roberts velocity profile, sometimes keeping walls in the simulation box and sometimes without them.

To introduce walls in our simulation box we use a relatively new method for flow simulation, the Lattice Boltzmann Method (LBM). We show here that LBM can work well as Navier-Stokes solver, and we have been able to reproduce the Taylor-Couette instability using this method.

Later the LBM code is coupled with the spectral code to have a complete non-linear dynamo code. The spectral part of this coupled code is utilized mainly to simulate the induction equation, whereas the LBM part works as a Navier-Stokes solver.

In an analytical calculation we show that the modified velocity profile due to alpha quenching can give rise to a wave whose frequency depends on the magnetic field as Alfvén wave but propagates perpendicular to the magnetic field. This calculation is based on an approximation where inertia of the fluid is neglected.

In one of our simulations we show the presence of subcritical dynamo onset for a dynamo which is non-linear, in contrast to the kinematic simulations.

Finally we show with our simulations that turbulence gives rise to a magnetic field oscillation. This newly developed oscillation has the same kind of $R_{m}$-dependencies as is observed in the real experiment, but the origin of this oscillation is again different from the wave we found using our analytical calculations.

The complex mechanical structures of the experiment can give rise to many interesting things, as for example the boundary layer near to the wall can behave differently. Our present simulations are done on rather coarse grids, which is still unable to resolve this boundary layer. Future fine-grid simulations can help us to study the complicated flow structure near to the wall-boundary and walls can also be made thinner which will be more realistic.

The energy dissipation calculation has still to be done. Variation of energy dissipation with magnetic Reynolds number $\left(R_{m}\right)$ can be checked with the experimental results.

As the LBM code has verified the Taylor-Couette instability it can be further extended to see the magnetic field effect on this kind of flow structure. Specially this flow is spatially periodic in nature. Hence it could give rise to the dynamo effect too.

At the end, the hybrid code can be used to simulate various kinds of MHD problems where specially developed complex boundaries are needed to be implemented for fluid flow. We hope that opens up new horizons in science. 



\section{A Appendix: A Mean field view of the Karlsruhe Dynamo}

To apply the mean-field concept in the G. O. Roberts kind of dynamo one assumes for each given field $\mathrm{F}$ that there is a mean field $\bar{F}$ by taking an average over an area corresponding to the cross-section of four cells (each having length and width $a$ ) in the $x y$ plane,

$$
\bar{F}(x, y, z)=\frac{1}{4 a^{2}} \int_{-a}^{a} \int_{-a}^{a} F(x+\chi, y+\kappa) d \chi d \kappa
$$

Splitting the magnetic flux density $\boldsymbol{B}$ and the fluid velocity $\boldsymbol{u}$ into mean fields $\overline{\boldsymbol{B}}, \overline{\boldsymbol{u}}$ and remaining fields $\boldsymbol{B}^{\prime}$ and $\boldsymbol{u}^{\prime}$ one gets

$$
\boldsymbol{B}=\overline{\boldsymbol{B}}+\boldsymbol{B}^{\prime}, \quad \boldsymbol{u}=\overline{\boldsymbol{u}}+\boldsymbol{u}^{\prime}
$$

But for the flow corresponding G. O. Roberts one has $\overline{\boldsymbol{u}}=0$ and hence $\boldsymbol{u}=\boldsymbol{u}^{\prime}$. Following this one has

$$
\begin{gathered}
\frac{\partial \overline{\boldsymbol{B}}}{\partial t}=\nabla \times \boldsymbol{\epsilon}+\frac{1}{\eta} \nabla^{2} \overline{\boldsymbol{B}} \\
\frac{\partial \boldsymbol{B}^{\prime}}{\partial t}=\nabla \times\left(\boldsymbol{u}^{\prime} \times \overline{\boldsymbol{B}}+\boldsymbol{u}^{\prime} \times \boldsymbol{B}^{\prime}-\epsilon\right)+\frac{1}{\eta} \nabla^{2} \boldsymbol{B}^{\prime}
\end{gathered}
$$

with $\eta=\mu \sigma$ and $\epsilon$ can be represented in the form

$$
\epsilon_{i}=\alpha_{i j} \bar{B}_{j}-\beta_{i j k} \frac{\partial \bar{B}_{k}}{\partial x_{j}} .
$$

Due to the definition of averages and the periodicity of the flow pattern $\alpha_{i j}$ and $\beta_{i j k}$ are independent of $x$ and $y . \quad \alpha_{i j}$ and $\beta_{i j k}$ are axisymmetric tensors with respect to the $z$-axis. Primarily $\boldsymbol{u}$ is independent of $z$ (unless one considers the boundary effect of the real experiment). Hence one arrives at

$\boldsymbol{\epsilon}=-\alpha_{\perp}(\overline{\boldsymbol{B}}-(\boldsymbol{e} \cdot \overline{\boldsymbol{B}}) \boldsymbol{e})-\beta_{\perp} \nabla \times \overline{\boldsymbol{B}}-\left(\beta_{\|}-\beta_{\perp}\right)(\boldsymbol{e} \cdot(\nabla \times \overline{\boldsymbol{B}})) \boldsymbol{e}-\beta_{3} \boldsymbol{e} \times(\nabla(\boldsymbol{e} \cdot \overline{\boldsymbol{B}})+(\boldsymbol{e} \cdot \nabla) \overline{\boldsymbol{B}}) ;$ and $z$.

with $\alpha_{\perp}, \beta_{\perp}, \beta_{\|}, \beta_{3}$ are averaged quantities determined by $\boldsymbol{u}$ and independent of $x, y$ 
The term $\alpha_{\perp}$ describes an alpha effect, which is extremely anisotropic. It is able to drive electric currents in the $x$ and $y$ direction. $\beta_{\perp}$ and $\beta_{\|}$are also anisotropic and give rise to the mean field diffusivity of the fluid, but $\beta_{3}$ cannot be interpreted as mean-field diffusivity since it is not the term connected with $\nabla \times \bar{B}$. 


\section{Bibliography}

[1] Arnold, V. I. , 1965, Sur la topologie des écoulements stationnaires des fluides parfaits, C. R. Acad. Sci., Paris, 261, 43-46

[2] Backus, G. E. and Chandrasekhar, S. , 1956, On Cowling's theorem on the impossibility of self-maintained axisymmetric homogeneous dynamos, Proc. Nat. Acad. Sci., 42, 105-109

[3] Beltrami, E. , 1889, Opera Matematiche, 4, 304

[4] Bhatnagar, P., Gross, E., Krook, M. , 1954, A model for collisional process in gases I: small amplitude processes in charged and neutral one-component system, Phys. Rev.,94, 511

[5] Breyiannis, G., Valougeorgis, D. , 2004, Lattice kinetic simulations in 3D Magnetohydrodynamics, Phys. Rev. E, 69, 065702

[6] Bullard, E. C. and Gellman, H. , 1954, Homogeneous dynamos and terrestrial magnetism, Phil. Trans. Roy. Soc., A 247, 213-278

[7] Busse, F. H. , 1971, Z. Geophys., 37, 153

[8] Busse, F. H. , 1977, J. Geophys, 43, 441

[9] Busse, F. H. , 1975, A model of the geodynamo, A model of the geodynamo, Geophy. J. R. Astron. Soc., 42, 437-459

[10] Busse, F. H. ,1992, Dynamo theory of planetary magnetism and laboratory experiments, In Fredrich, R., Wunderlin, A. (eds.), Proceedings in Physics, 59, 197-207

[11] Busse, F. H. , 2000, Homogeneous Dynamos in Planetary Cores and in the Laboratory, Ann. Rev. Fluid Mech., 32, 383-408

[12] Childress, S. , 1967, Construction of steady-state hydromagnetic dynamos. I. Spatially periodic fields, Report MF-53, Courant Institute of Mathematical Sciences

[13] Childress, S. , 1970, New solutions of the kinematic dynamo problem, J. Math. Phys., 11, 3063-3076

[14] Chen, H., Kandasamy, S., Orszag, S., Shock, R., Succi, S., Yakhot, V., 2003 , Extended Boltzmann Kinetic Equation for Turbulent Flows, Science, 301, 633-636 
[15] Christensen, U. R. and Tilgner, A. , 2004, Power requirement of the geodynamo from Ohmic losses in numerical and laboratory dynamos, Nature, 429, 169-171

[16] Cowling, T. G. , 1934, The magnetic field of sunspots, Mont. Not. R. Astron. Soc., $94,39-48$

[17] Dellar, P. , 2001, Bulk and shear viscosities in lattice Boltzmann equations, Phys. Rev. E, 64, 031203

[18] Dellar, P. , 2002, Lattice kinetic schemes for magnetohydrdynamics, J. Comput. Phys., 179, 95-126

[19] Elsasser, W. M. , 1946, Induction effects in terrestrial magnetism, Phys. Rev., 69, 106-116

[20] Finlayson, B. A. and Seriven, L. E. , 1966, Galerkin's method and the local potential, net.. Conf.., 291

[21] Frankel, Ya. I. , 1945, Russian Journal, 49, 98-101

[22] Gailitis, A. , 1967, Russian Publication, 3, 45-54

[23] Gailitis, A., Lielausis, O., Platacis, E., Gerbeth, G., Stefani, F. , 2003, The Riga Dynamo Experiment, surveys in Geophysics 24, 247-267

[24] Ginzbourg, I. and d'Humiŕes, D. , 1996, Local Second-Order Boundary Methods for Lattice Boltzmann Models, J. Stat. Phys., 84, 927-971

[25] Glatzmaier, G. A., Roberts, P. H. , 1995a, A three dimensional self-consistent computer simulation of a geomagnetic field reversal, Nature, 377, 203-209

[26] Glatzmaier, G. A., Roberts, P. H. , 1995b, A three-dimensional convective dynamo solution with rotating and finitely conducting inner core and mantle, Phys. Earth Planet. Inter., 91, 63-75

[27] He, X., Shan, X., Doolen, G. , 1998, Discrete Boltzmann equation model for nonideal gases, Phys. Rev. Lett., 57, R13-R16

[28] Moffat, H. K. , 1969, The degree of knottedness of tangled vortex lines, J. Fluid Mech., 35, 117-129

[29] Moffat, H. K. , 1970, Turbulent dynamo action at low magnetic Reynolds number, J. Fluid Mech., 41, 435-452

[30] Müller U., Stieglitz R., and Horanyi S. , 2004, A two-scale hydromagnetic dynamo experiment, Jour. Fluid. Mech., 498, 31-71

[31] Kandanoff, L. , 1986, On two levels, Phys. Today, 39, 7-9

[32] Krause, F. and Rädler, K. -H , 1980, Mean-Field Magnetohydrodynamics and Dynamo Theory, Aksdemie-Verlag, Berlin 
[33] Kreiss, H. O., Oliger, J. , 1972, Comparison of accurate methods for the integration of hyperbolic equations, Tellus, 24, 199

[34] Ladd, A. J. C. , 1994, Numerical simulations of particulate suspensions via a discretized Boltzmann equation, Part 1. Theoretical foundation, j. Fluid. Mech., 271, 285-309

[35] Lallemand, P., Luo, L.-S. , 2003, Theory of the lattice Boltzmann method: Acoustic and thermal properties in two and three dimensions, Phys. Rev. E 68(3): 036706

[36] Lamor, J., 1919, How could a rotating body such as the sun become a magnet?, Br. Assoc. Adc. Sci. Rep., 159-160

[37] Lehnert, B. , 1958, Ark. Fys. 13, 10, 109

[38] Lortz, D. , 1968, Impossibility of steady dynamos with certain symmetries, Phys. Fluids, 11, 913-915

[39] Lowes, F. J. and Wilkinson, I. , 1963, Geomagnetic dynamo: a laboratory model, Nature, 198, 1158-1160

[40] Lowes, F. J. and Wilkinson, I. , 1968, Geomagnetic dynamo: an improved laboratory model, Nature, 219, 717-718

[41] Luo, L.-S. , 1998, A Unified Theory of Non-Ideal Gas Lattice Boltzmann Models, Phys. Rev. Lett., 81, 1618-1621

[42] Marie, L., Burguette, J., Chiffaudel, A., Daviaud, F., Ericher, D., et al. , 2001, MHD in von Karman swirling flows, Dynamo and Dynamics, a Mathematical challenge (ed. D. Armbruster \& J. Opera), Nato Science Series, 26

[43] Müller, U. and Stieglitz, R. , 2002, The Karlsruhe Dynamo Experiment, nonlin. Proc. in Geophys., 9, 165-170

[44] Müller, U., Stieglitz, R., Horanyi, S. , 2004, A two-scale hydromagnetic dynamo experiment, 498, 31-71

[45] Parker, E. N. , 1955, Hydromagnetic dynamo models, Astrophys. J., 122, 293-314

[46] Peffley, N. L., Cawthorne, A. B. and Lathrop, D. P. , 2000, Toward a self generating dynamo: The role of turbulence. Phys. Rev. E, 61, 5287-5296

[47] Ponomarenko, Yu. B., 1973 Zh. Prikl. Mekh. Tekhn. Fiz., 6, 47-51

[48] Pouquet, J., Frisch, U., Leorat, J. , 1976, Strong MHD helical turbulence and the non-linear dynamo effect, J. Fluid Mech., 77, 321-354

[49] Rädler K.-H., Apstein E., Rheinhardt M., Schüler M. , 1998, StGG. 42, 224-231

[50] Rädler K.-H., Rheinhardt M., Apstein E., and Fuchs H. , 2002a On the mean-field theory of the Karlsruhe Dynamo Experiment, Nonlin. Phys. Geo, 9, 171-187 
[51] Rädler K.-H., Rheinhardt M., Apstein E., Fuchs,H , 2002b, On the mean-field theory of the Karlsruhe Dynamo Experiment II. Back-reaction of the magnetic field on the fluid flow, Magnetohydrodynamics, 38, 73-94

[52] Rädler K.-H. and Brandenburg A. , 2003, Contributions to the theory of a two-scale homogeneous dynamo experiment, Phys. Rev.E, 67, 026401

[53] Roberts, G. O. , 1972, Dynamo action of fluid motions with two-dimensional periodicity, Phil. Trans. Roy. Soc. A, 271, 411-454

[54] Rüdiger, G. and shalybkov, D. A. , 2001, MHD instability in cylindric TaylorCouette flow, international Couette-Taylor Workshop

[55] Sarkar, A. and Tilgner, A. , 2005a, Simulation of the dynamo effect with the lattice Boltzmann method, Comp. Fluid., in press

[56] Sarkar, A. and Tilgner, A. , 2005b, Magnetic field oscillation in simulations of the Karlsruhe Dynamo, Astron. Nachr., 326, 250-253

[57] Steenbeck, M., Krause, F., Rädler, K. -H , 1966, Berechnung der mittleren LorentzFeldstärke $\overline{\boldsymbol{v} \times \boldsymbol{B}}$ für ein elektrich leitendes Medium in turbulenter, durch CoriolisKräfte beeinflusster Bewegung. Z. Naturforch, 21a, 369-376

[58] Steenbeck, M., Kirko, I. M., Gailitis, Klawina, A., P., Krause, F., Laumanis, I. J. , 1967, Der experimentelle Nachweis einer electromotorichen Kraft längs eines äusseren Magnetfeldes, induziert durch eine Strömung flüssigen Metals (alpha Effekt), Mber. dtsch. Akad. Wiss. Berlin, 9, 714-719

[59] Stieglitz, R. and Müller, U., 2001, Phys. Fluid., 13, 561-564

[60] Tilgner, A., 1997, A kinematic dynamo with a small scale velocity field, Phys. Lett. A., 226, 75-79

[61] Tilgner, A. , 2002, Numerical simulation of the onset of dynamo action in an experimental two-scale dynamo, Phys. Fluid., 14, 4092-4094

[62] Tilgner, A. and Busse, F. H. , 1995 Proc. R. Soc. Lond. A, 448, 237-244

[63] Tilgner, A. and Busse, F. H. , 2001 In P. Chossat, D. Armbruster, and I. Oprea, editors, Dynamo and Dynamics, a Mathematical Challenge. Kluwer

[64] Tilgner, A. and Busse, F. H. , 2002 Magnetohydrodynamics, 38, 35-40

[65] Vainshtein, S. I. and Zeldovich, Ya. B. , 1972, Origin of magnetic fields in astrophysics, Sov. Phys. Usp., 15, 159-172

[66] Zeldovich, Ya. B., Ruzmaikin, A. A. and Sokoloff, D. D., , 1983, The fluid mechanics of astrophysics and geophysics, vol. 3, Gordon \& Breach 


\section{Acknowledgements}

Prof. Andreas Tilgner, my supervisor, had played a significant role in the completion of this thesis. He suggested me this interesting problem. His generous supervision, actually, made me feel comfortable in pursuing the subject. During the three years of my work he always had time for discussion, refusing gracefully to discriminate between my questions, apt and silly. I just lack pat words to thank Prof. Tilgner, my mentor.

Very special thanks to Dr Dieter Schmitt, the International Max-Planck Research School (IMPRS) co-ordinator, for his care and support during the past three years.

I am appreciative of so many joyful moments I shared with my colleagues at the Göttingen Geophysical Institute. I acknowledge special thanks to Tomi, Stefan Bernhard, Ellen, Jan, Arno, Ole, Thomas Hartlap; and very special thanks to Frau Eggert-Heise, Prof. Bahr, Ulrich Einecke, Rainer and Manfred. Thanks are also due to Prof. Schmucker who was very friendly and spent much of his time for me.

Wherever I am in future, it will be hard for me to forget the Lindau Indian community. Dr Tripathi had made my life easy with his fondness for talking and jovial nature; Rupali was caring and supportive and sacrificed much of her time for me; Dilip and Rajat were always friendly.

I am very thankful to Dr Ajay Manglik for helping me to settle in a new country at the beginning. My friends Dr TRS and Viju deserve special thanks for being with me whenever I needed them.

The Max-Planck, Lindau Dynamo and fluid dynamics group deserves special thanks. Dr Wicht and Dr Aubert were always ready for friendly discussion, regardless of science or politics. The Lindau IMPRS group with fun, travel and academic discussions made my stay here comfortable. Along with all others I thank specially Martin Schrinner, Monika, Mark, Merilena and Denise.

I would like to thank Mr. Ramesh Chakrabarty for the long time he spent in correcting the manuscript. Dr Naresh Mitra, without whom I would never have been in Physics, deserves special mention.

The financial support from the Max-Planck Research School and "Graduiertenkolleg Strömungsinstabilitäten und Turbulenz" an der Universität Göttingen are thankfully acknowledged.

My sister Sarmila deserves credit for supporting me whenever I needed her.

My final words are dedicated to my parents. Their encouragement, support and good wishes made me what I am today. 



\section{Scientific Contributions}

\section{Publications:}

- Sarkar, A. and Tilgner, A., 2005, Simulation of the dynamo effect with the lattice Boltzmann method, Comp. Fluid., in press

- Sarkar, A. and Tilgner, A. , 2005, Magnetic field oscillation in simulations of the Karlsruhe Dynamo, Astron. Nachr., 326, 250-253

\section{Conference contributions:}

- On the oscillation of the Karlsruhe Dynamo Experiment: Freiburg, Germany, Dynamos of the Sun, Stars and Planets. 



\section{Lebenslauf}

$\begin{array}{ll}\text { Name: } & \text { Aveek Sarkar } \\ \text { Geburtsdatum: } & 26.10 .1974 \\ \text { Geburtsort: } & \text { Calcutta, India } \\ \text { Eltern: } & \text { Dr Arunendu Sarkar } \\ & \text { Mrs. Keya Sarkar } \\ \text { Familienstand: } & \text { ledig } \\ \text { Staatsangehörigkeit } & \text { Indian }\end{array}$

Schulbildung: $\quad$ 1/1981 bis 6/1993 Hindu School,

Calcutta, India

$\begin{array}{lll}\text { Studium: } & \text { B.Sc., } & \\ & & \begin{array}{l}\text { Vidyasagar College, } \\ \text { University Of Calcutta, } \\ \text { Calcutta, India }\end{array} \\ & \\ 1998 \text { bis } 2000 & \text { M.Sc., } & \begin{array}{l}\text { Pune University, } \\ \text { Pune,India }\end{array}\end{array}$

Tätigkeiten: Aug. 2000 bis Mai. $2001 \quad$ National Center for Radio Astrophysics, Pune, India

Juli 2001 bis Jan. $2002 \quad$ Indian Space Research Organisation, Thiruvanthapuram, India

Promotion: $\quad$ Feb. 2002 bis Juli $2005 \quad$ Promotion am Institut für Geophysik der Universität Göttingen 